\title{
AN INVESTIGATION OF AVIAN WING TIP VORTEX GENERATION USING A BIOMIMETIC APPROACH
}

\author{
A Thesis \\ presented to \\ the Faculty of California State Polytechnic University, \\ San Luis Obispo
}

\author{
In Partial Fulfillment \\ of the Requirements for the Degree \\ Master of Science in Aerospace Engineering
}

by

David S. Martin

June 2017 
(C) 2017

David S. Martin

ALL RIGHTS RESERVED 


\section{COMMITTEE MEMBERSHIP}

TITLE:

An Investigation of Avian Wing Tip Vortex

Generation Using a Biomimetic Approach

AUTHOR: $\quad$ David S. Martin

DATE SUBMITTED: June 2017

COMMITTEE CHAIR: Graham Doig, Ph.D.

Assistant Professor of Aerospace Engineering

COMMITTEE MEMBER: Aaron Drake, Ph.D.

Associate Professor of Aerospace Engineering

COMMITTEE MEMBER: David Marshall, Ph.D.

Aerospace Engineering Department Chair

COMMITTEE MEMBER: Clinton Francis, Ph.D.

Assistant Professor of Biological Sciences 


\begin{abstract}
An Investigation of Avian Wing Tip Vortex Generation Using a Biomimetic Approach
\end{abstract}

David S. Martin

An experimental study has been conducted to develop a process allowing the creation of biologically accurate aerodynamic test models mimicking the slotted primary feather geometry of the Brown Pelican (Pelecanus occidentalis). Preserved examples of both a full Brown Pelican wing and a single primary feather were $3 \mathrm{D}$ scanned and digitally reconstructed using a combination of MATLAB and CAD software. The final model was then 3D printed as a collection of smaller components using a LulzBot TAZ 6 printer and Taulman3D T-Glase PET filament. After using various surface finishing techniques to improve the finish of all 3D printed parts, an assembly was designed to mount the model in the low speed wind tunnel at the California Polytechnic State University. Prior to aerodynamic testing, airfoil sections of the pelican wing were generated in $\mathrm{CAD}$ and several common airfoil measurements and characteristics were investigated. At a flow velocity of $5 \mathrm{~m} / \mathrm{s}\left(\operatorname{Re} \sim 1.21 \times 10^{5}\right)$, wind tunnel smoke and laser visualization testing highlighted the vortex generation of multiple primary feathers, as well as large-scale flow deviations in the vicinity of the feathers. A total pressure rake and total pressure probe were used to create detailed plots of the ratio of the local velocity to free-stream velocity $\left(\mathrm{V}_{\mathrm{x}} / \mathrm{V}_{\mathrm{x} \infty}\right)$ at two planes downstream of the model, which revealed vortex positioning consistent with that predicted by smoke visualization testing and provided a metric by which to evaluate the relative strength of each vortex.

The model creation process and wind tunnel testing results outlined here provide a strong foundation for future investigations into the potential aerodynamic benefits provided by the slotted primary feather geometry employed by the Brown Pelican and other large gliding avian species.

Keywords: Pelecanus occidentalis, Brown Pelican, biomimicry, 3D printing, wind tunnel 


\section{ACKNOWLEDGMENTS}

I would like to thank, first and foremost, Dr. Graham Doig for his guidance throughout the research and thesis writing process. Graham was always willing to lend his wealth of experience and knowledge and provided valuable insight during all portions of the research process. This work would not have been possible without his encouragement and for that I am sincerely grateful.

Thank you to the Los Angeles Natural History Museum for the gracious loaning of their time and resources and allowing our research team to 3D scan several avian specimens. Many thanks to Dr. Krista Fahy and the Santa Barbara Museum of Natural History for their willingness to loan a Brown Pelican primary feather for 3D scanning and aerodynamic testing. I would also like to acknowledge the BBC's contribution of video footage for this project.

A big thank you to Cody Thompson for helping to create the machined components for the final aerodynamic test model and to Brandon Baldovin, Lucas Dodd and Lucas Payne for their help in conducting this research. Their contributions to the 3D scanning, 3D printing and fabrication processes were invaluable and much appreciated.

Finally, I would like to thank my wife Casey for her strength and patience during my career as a Cal Poly graduate student, as well as my parents for their constant encouragement and support. 


\section{TABLE OF CONTENTS}

Page

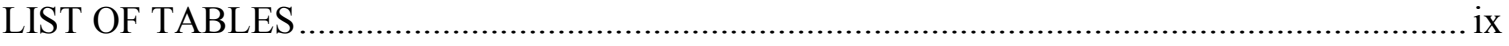

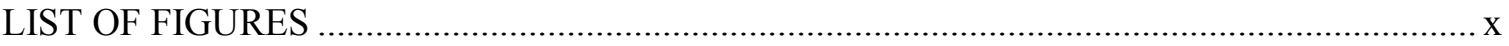

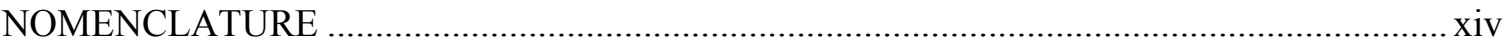

\section{CHAPTER}

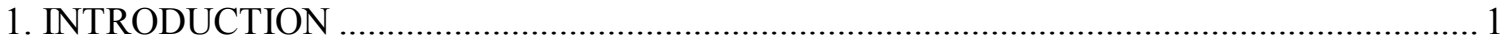

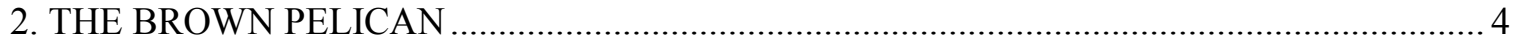

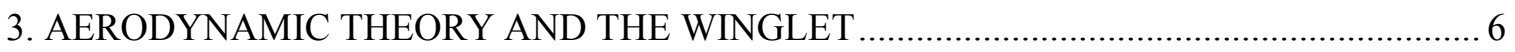

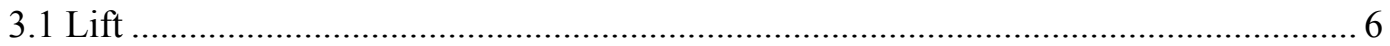

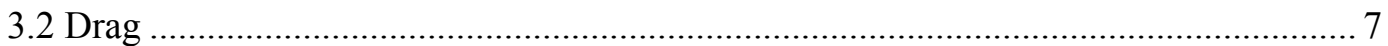

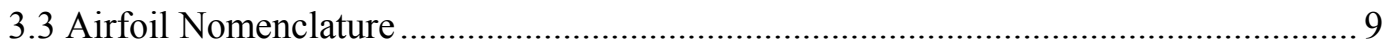

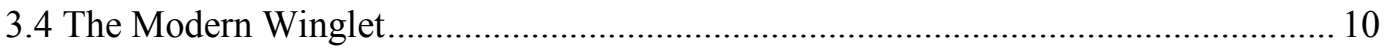

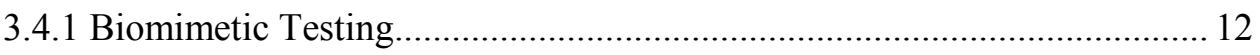

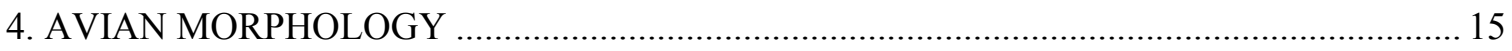

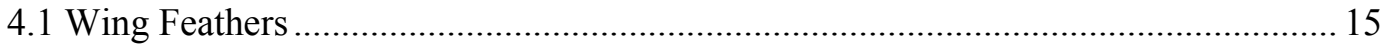

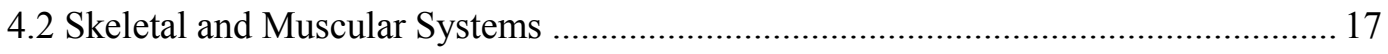

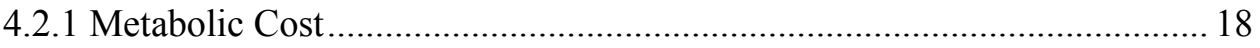

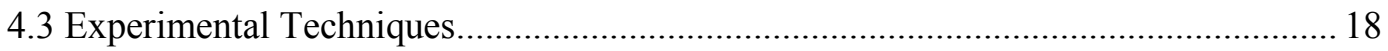

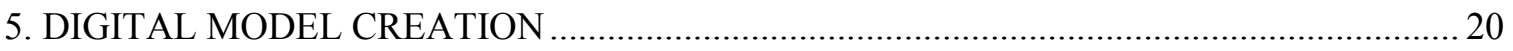

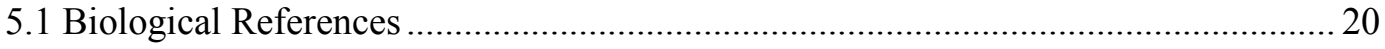

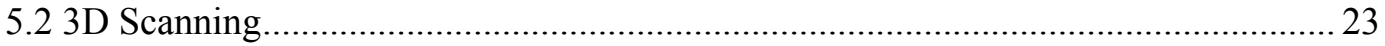

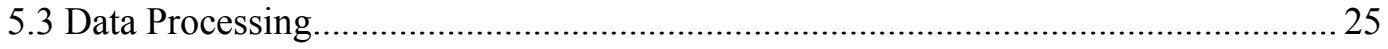

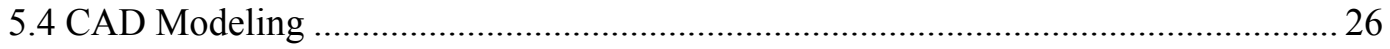




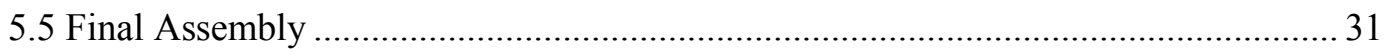

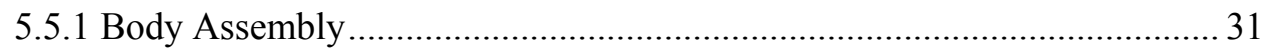

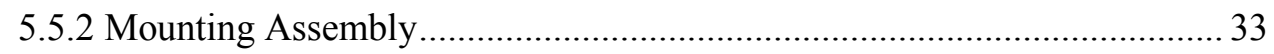

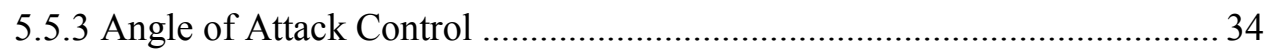

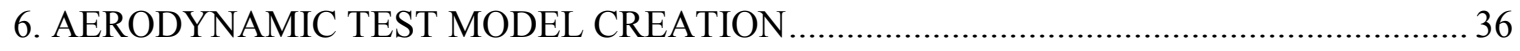

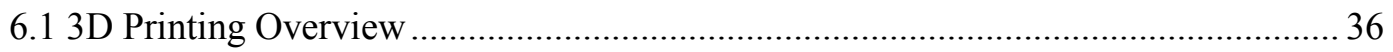

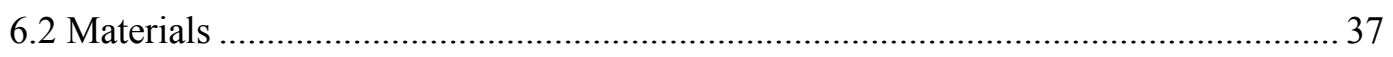

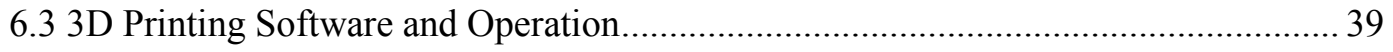

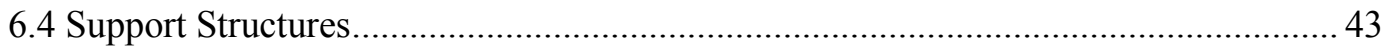

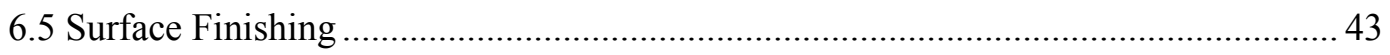

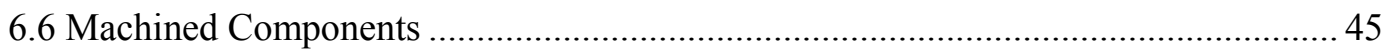

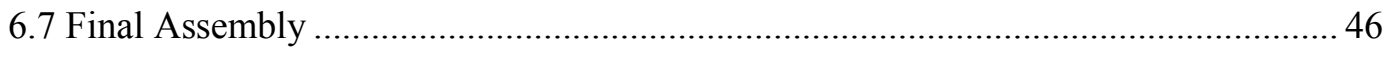

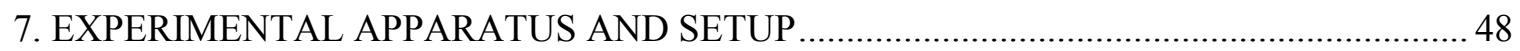

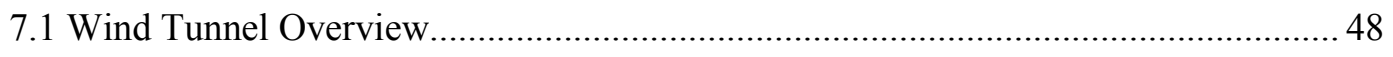

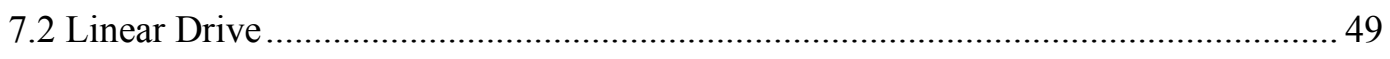

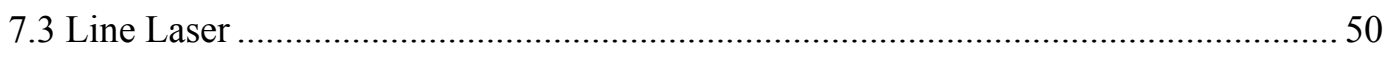

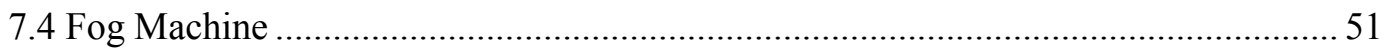

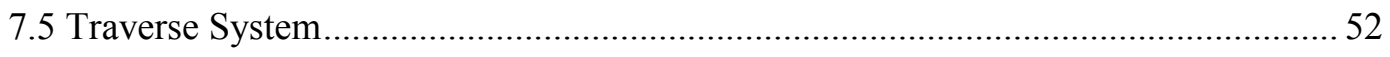

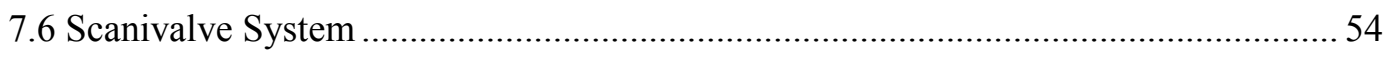

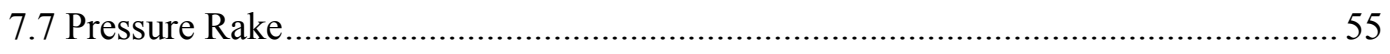

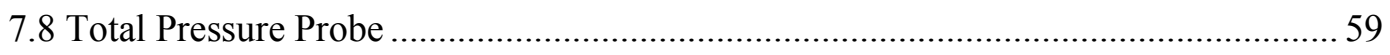

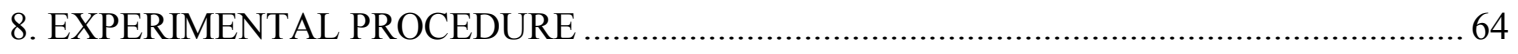

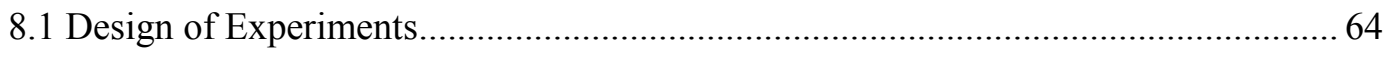

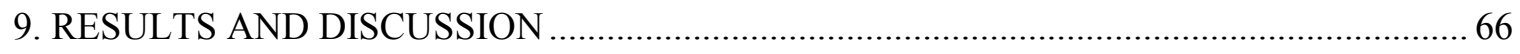

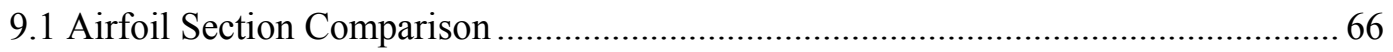




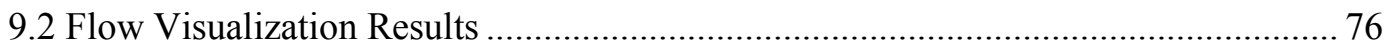

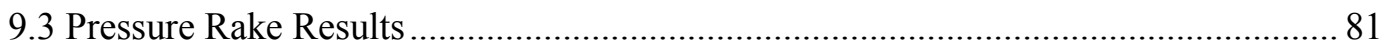

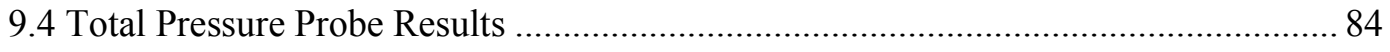

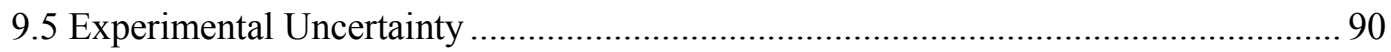

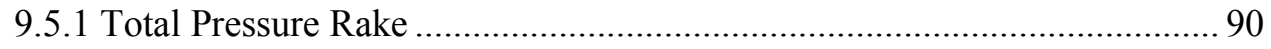

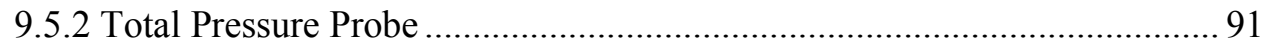

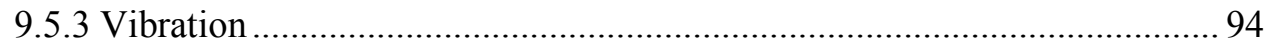

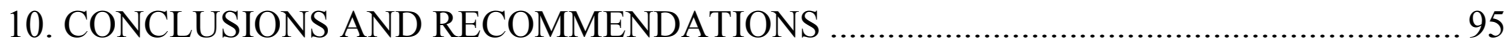

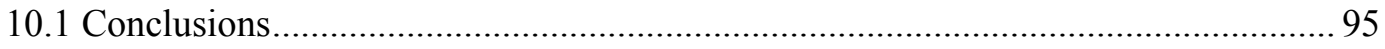

10.2 Simplifications and Recommendations for Future Work ...................................... 97

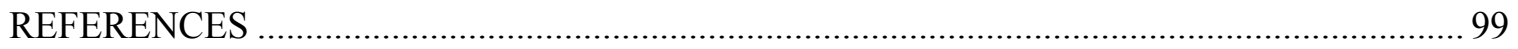

APPENDICES

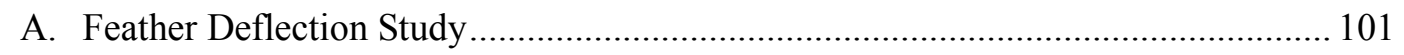

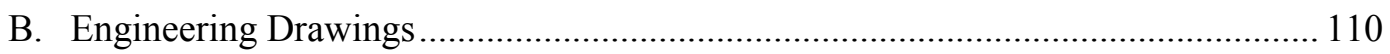

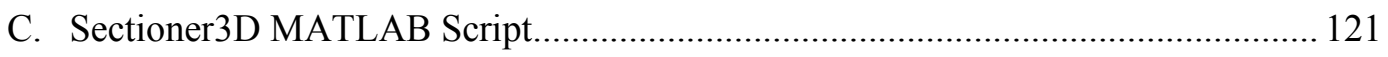

D. Total Pressure Rake Post-Processing MATLAB Script ......................................... 123

E. Total Pressure Probe Post-Processing MATLAB Script - Example ........................ 128 


\section{LIST OF TABLES}

Table

Page

1. Scaling factor and chord angle of each airfoil section with respect to that of section 1

2. Airfoil data for sections of the 3D scanned Brown Pelican wing . .68

3. Angle of attack of each primary feather measured at the mid-span with reference to the chord of wing section 1 .. 76 


\section{LIST OF FIGURES}

Figure

Page

1. A juvenile Brown Pelican in flight near Bodega Bay, California ${ }^{6}$.......................................... 4

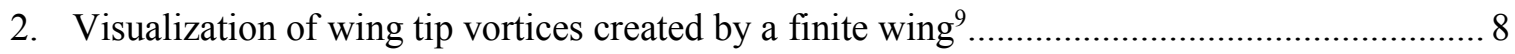

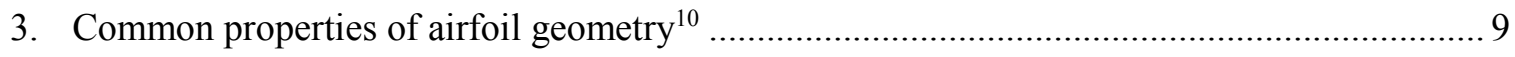

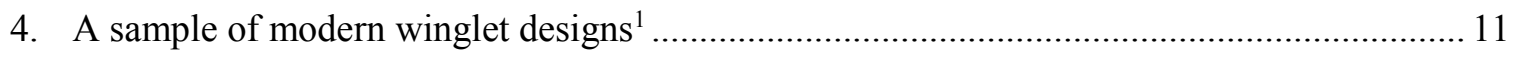

5. Dragon Eye UAV with cascading winglet geometry investigated by Shelton et al. ${ }^{16}$........... 13

6. Morphology of the primary feathers of a Brown Pelican in order from the leading edge (left) to the trailing edge (right) of the right wing ${ }^{24}$ 16

7. Overview of the Brown Pelican wing structure presented by Simons et al. Labels have been added for clarity ${ }^{22}$ 17

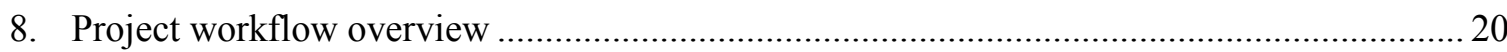

9. Brown Pelican primary feather loaned by the Santa Barbara Museum of Natural History..... 21

10. 3D scanning of a Brown Pelican wing at the Los Angeles Natural History Museum. Visible on the floor is the preserved wing of a California Condor.

11. CAD surfaces used to determine the general size and curvature of the primary feathers on the final model.

12. 3D scans of a Brown Pelican wing and primary feather generated by the NextEngine 3D scanner (not to scale)

13. Highlighted airfoil sections generated by the MATLAB script overlaid on the primary

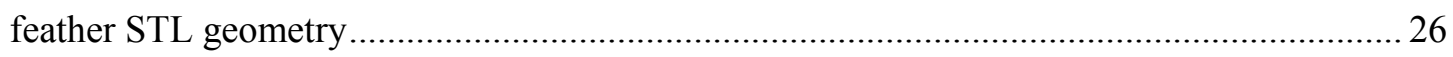

14. Comparison of the primary feather a) construction geometry and b) solid geometry ............. 28

15. Comparison of the wing body a) construction geometry and b) solid geometry.................... 29

16. Detail of the final scaled wing geometry. View from the rear ............................................ 31

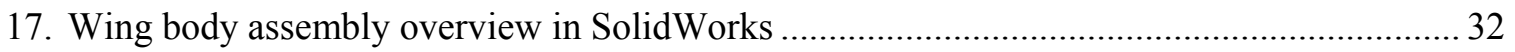


18. Mounting assembly overview with linear drive mounting plate shown (top).....

19. Overview of the final model at a) $0^{\circ}$, b) $5^{\circ}$ and c) $10^{\circ}$ angle of attack. Air flow is from top to bottom

20. A comparison between identical parts printed in ABS (left) and T-Glase (right). Warping and splitting between print layers is clearly visible in the ABS print 39

21. Detail views of the support structures designed for each feather component

22. Final aerodynamic test model mounted in the Cal Poly low speed wind tunnel. Side view ... 47

23. Final aerodynamic test model mounted in the Cal Poly low speed wind tunnel. Rear view... 47

24. The Cal Poly low speed wind tunnel with the test section doors open. Air flow is from left to right 48

25. Overview of the linear drive system with mounting plate extended 50

26. Line laser setup. .51

27. Fog machine setup. 52

28. Measurement device mounting assembly .53

29. Overview of the traverse system with no measurement devices mounted 54

30. ERAD4000 LabVIEW interface. .55

31. Pressure rake mounted to the traverse arm. Pressure tubing is routed across the arm of the traverse to the Scanivalve box (blue and yellow) shown in the background...

32. Pressure rake measurement grid a) looking forward from downstream of the model and b)

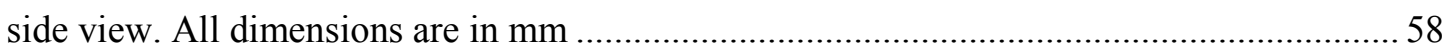

33. Total pressure probe setup.

34. First total pressure probe measurement grid a) looking forward from downstream of the model and b) side view. All dimensions are in $\mathrm{mm}$

35. Second total pressure probe measurement grid a) looking forward from downstream of the model and b) side view. All dimensions are in $\mathrm{mm}$ 63 
36. Simplified airfoil sections along the span of the Brown Pelican wing.

37. Simplified airfoil sections from the wing of a Brown Pelican compared to sections from an Australian Pelican in a thermal soaring configuration generated by Traill. ${ }^{9}$ For visual reference only, not to scale.

38. Comparison of airfoil characterization data between the Brown Pelican wing and Australian Pelican wing in a thermal soaring configuration generated by Traill. ${ }^{9}$.

39. Simplified airfoil sections from the wing of a Brown Pelican compared to sections from an Australian Pelican in a ground effect configuration generated by Traill. ${ }^{9}$ For visual reference only, not to scale.

40. Comparison of airfoil characterization data between the Brown Pelican wing and Australian Pelican wing in a ground effect configuration generated by Traill. ${ }^{9}$

41. Still frame detailing relative size and location of vortices downstream of the test model. Flow velocity $5 \mathrm{~m} / \mathrm{s}$, angle of attack $10^{\circ}$

42. Collection of still frames showing vortex development from the $1^{\text {st }}, 2^{\text {nd }}$ and $4^{\text {th }}$ primary feathers. Flow velocity $5 \mathrm{~m} / \mathrm{s}$, angle of attack $10^{\circ}$

43. Flow deflection around the primary feathers. Side view

44. Plot of the velocity ratio $\mathrm{V}_{\mathrm{x}} / \mathrm{V}_{\mathrm{x} \infty}$ generated using a pressure rake at a distance of $0.2 \mathrm{~m}$ downstream of the model and a flow velocity of $5 \mathrm{~m} / \mathrm{s}$.

45. Comparison of the $\mathrm{CAD}$ geometry to data generated during pressure rake testing with the sampling plane located $0.2 \mathrm{~m}$ downstream of the model. Each vortex structure and its generating feather are matched by number. All dimensions are in $\mathrm{mm}$

46. Plot of the velocity ratio $\mathrm{V}_{\mathrm{x}} / \mathrm{V}_{\mathrm{x} \infty}$ generated using a total pressure probe at a distance of $0.2 \mathrm{~m}$ downstream of the model and a flow velocity of $5 \mathrm{~m} / \mathrm{s}$ 85 
47. Comparison of the CAD geometry to data generated during total pressure testing with the sampling plane located $0.2 \mathrm{~m}$ downstream of the model. Each vortex structure and its generating feather are matched by number. All dimensions are in $\mathrm{mm}$. .86

48. Plot of the velocity ratio $\mathrm{V}_{\mathrm{x}} / \mathrm{V}_{\mathrm{x} \infty}$ generated using a total pressure probe at a distance of $0.1 \mathrm{~m}$ downstream of the model and a flow velocity of $5 \mathrm{~m} / \mathrm{s}$ .88

49. Comparison of the CAD geometry to data generated during total pressure testing with the sampling plane located $0.1 \mathrm{~m}$ downstream of the model. Each vortex structure and its generating feather are matched by number. All dimensions are in $\mathrm{mm}$.....

50. Contour plot of the standard deviation of each data sample collected by the total pressure rake.

51. Contour plot of the standard deviation of each data sample collected by the total pressure probe at a distance of $0.1 \mathrm{~m}$ downstream

52. Contour plot of the standard deviation of each data sample collected by the total pressure probe at a distance of $0.2 \mathrm{~m}$ downstream 


$\begin{array}{ll}\text { English Symbols } \\ \text { Symbol } & \text { Definition } \\ \text { a } & \text { local speed of sound } \\ { }^{\circ} \mathrm{C} & \text { degrees Celsius } \\ \mathrm{cm} & \text { centimeters } \\ \mathrm{HP} & \text { horsepower } \\ \text { in } & \text { inches } \\ \mathrm{L} & \text { characteristic length } \\ \mathrm{M} & \text { Mach number } \\ \mathrm{m} & \text { meters } \\ \mathrm{mm} & \text { millimeters } \\ \mathrm{P} & \text { static pressure } \\ \mathrm{Pa} & \text { Pascals } \\ \mathrm{V} & \text { pounds per square inch } \\ \mathrm{V} & \text { Reynolds number } \\ \mathrm{V} & \text { free-stream flow velocity } \\ \mathrm{V} & \end{array}$

Greek Symbols

$\alpha \quad$ angle of attack (degrees)

$\rho \quad$ density

$\mu \quad$ dynamic viscosity 


\section{INTRODUCTION}

Minimizing aerodynamic drag, the resistive force created when an object moves through the air, has been the source of extensive research since the introduction powered flight in the early $20^{\text {th }}$ century. Drag effects can generally be split into three main categories: parasitic drag, wave drag and induced drag. Induced drag, or the drag associated with the creation of lift, is created when the high pressure air below the wing of an aircraft curls over wing tip into the region of low pressure above the wing, creating a strong recirculating region of low pressure air at the wing tip that disrupts the airflow around the wing. This reduces the wing's efficiency and increases the total fuel burn of the aircraft. Induced drag has been shown to account for approximately $40 \%$ of the total drag experienced by a subsonic transport aircraft during cruise conditions and up to $80-90 \%$ in take-off configuration. ${ }^{1}$ Additionally, these vortex structures may persist and remain airborne for up to several minutes and can create hazardous flight conditions for smaller aircraft in extreme cases. One common method to combat the creation of these wing tip vortices is through the use of winglets at the tip of the wing.

While many varied winglet designs currently exist, the first examples of modern winglets were inspired in large part by the wing tip primary feathers of large gliding avian species. ${ }^{2}$ Many species of large gliding avians, such as the Brown Pelican (Pelecanus occidentalis), California Condor (Gymnogyps californianus) and Turkey Vulture (Cathartes aura) exhibit complex wing tip feather geometries composed of multiple slotted primary feathers. It has previously been theorized that these slotted primary feathers play a role in the reduction of drag by breaking a single wing tip vortex into multiple smaller vortices, thereby reducing the overall intensity. These prominent flight feathers may also play a part in noise reduction, as well as avian yaw and roll control. However, testing these hypotheses has proven to be extremely difficult in practice, primarily due to the challenges related to conducting parametric studies in a controlled testing environment with birds of this size. While numerous studies investigating the flight characteristics of smaller avian species 
have been carried out in controlled environment (e.g. a wind tunnel or long hallway), additional studies have instead attempted to recreate the complex geometry of these splayed, cascading wing tip feathers. Such studies have met with varying levels of success. Simplifications often lead to stiff models composed of extruded airfoil sections protruding from the end of a wing without optimization, leading to vague approximations of the original geometry.

While obtaining aerodynamic data directly from a live bird would be ideal, this is often difficult in practice and makes a true parametric study extremely challenging. Alternatively, studies using recreated geometries are much more straightforward, but sacrifice realism as a result. This study attempts to resolve this knowledge gap by uniting the complex geometry of true avian wingtips with the thoroughness and relative ease of a parametric investigation by employing biomimetic principles. The foundation of this approach is comprised of $3 \mathrm{D}$ scanning and $3 \mathrm{D}$ printing technologies, as well as computer-aided design tools, all of which enable replication of complex biological structures with a high degree of accuracy when used in conjunction. The specific goals of this study are twofold:

1.) Develop a process to create biologically accurate aerodynamic test models of avian wing tip geometry.

2.) Evaluate the flow field around a test model using the California Polytechnic State University low speed wind tunnel and related systems.

Aircraft winglets can offer significant advantages when properly designed, ranging from lower operational costs and reduced emissions to less aerodynamic noise and higher cruise speeds. A winglet geometry accurately replicating the slotted primary feather geometry of large gliding avian species may offer additional benefits beyond those provided by modern winglets designs. Using 3D scan data collected from a Brown Pelican wing and primary feather, this study aims to 
lay the groundwork necessary for future investigation into the potential benefits of biomimetic winglet geometries. 
The Brown Pelican (Pelecanus occidentalis) is the smallest of the eight pelican species contained in Family Pelecanidae. In accordance with the species name occidentalis (Latin for "of/pertaining to the west"), the Brown Pelican is typically found in the western hemisphere along the coasts of North and South America. Though once endangered due to the use of organochlorine pesticides in North America, Brown Pelicans are now a relatively common sight along coastal areas. ${ }^{3,4}$ While awkward on land, the large wingspan of this bird (typically ranging from 1.8 to 2.5 $\mathrm{m}$ for males) permits efficient flight to and from feeding areas as well as breeding grounds, which are often located on small, uninhabited offshore islands. ${ }^{4}$ As foraging may occur up to $75 \mathrm{~km}$ from the nest, Brown Pelicans spend a significant amount of time airborne. ${ }^{4}$ Overall lengths of male specimens can range from 1.0 to $1.4 \mathrm{~m}$, whereas total weight may vary anywhere from 2.0 to 5.0 kg. ${ }^{4,5}$ Females are somewhat smaller than males with wings that are approximately $3-6 \%$ shorter. ${ }^{4}$

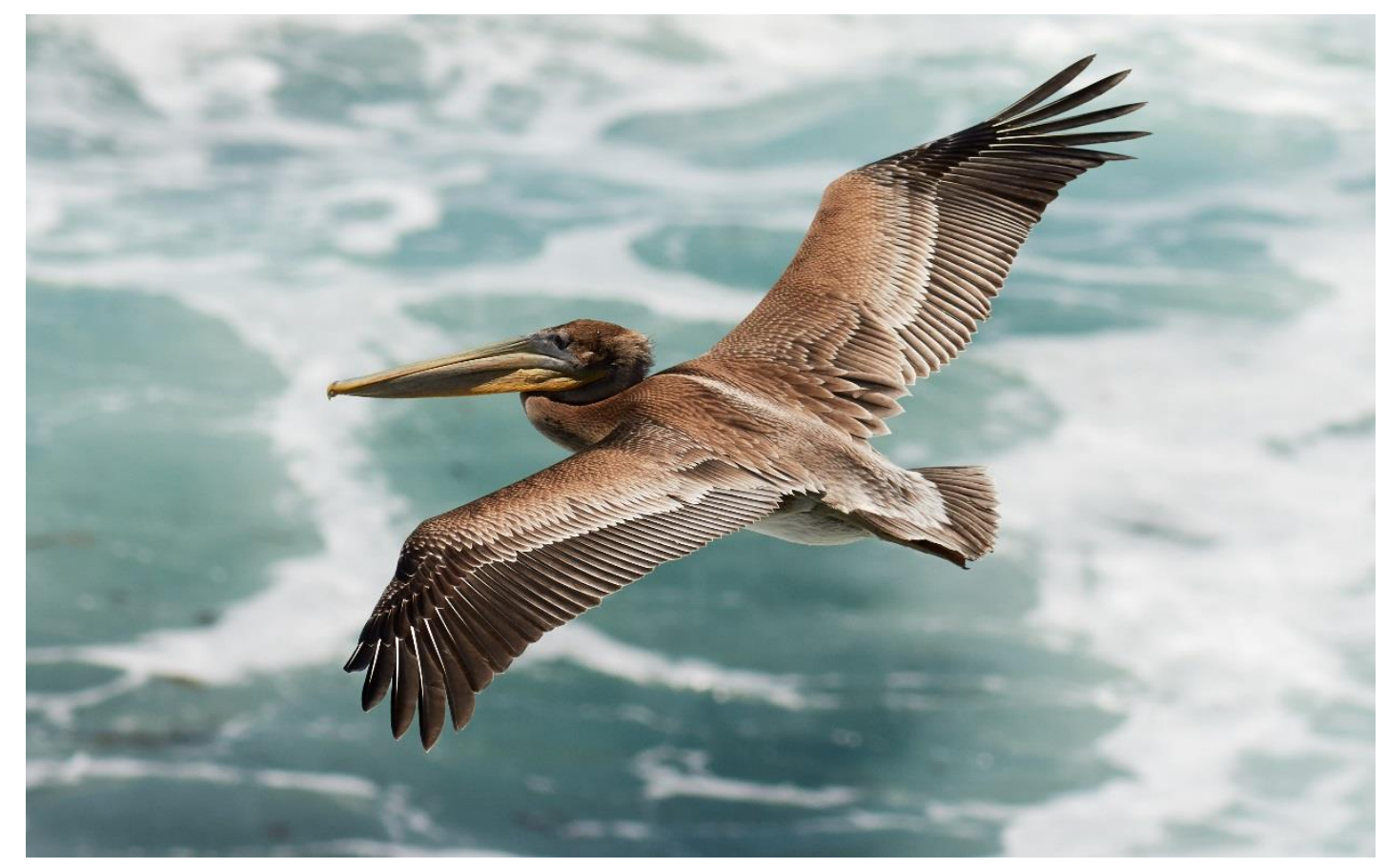

Figure 1. A juvenile Brown Pelican in flight near Bodega Bay, California. ${ }^{6}$ 
While the vast majority of the pelican diet is composed of small fish and crustaceans, Brown Pelicans are the only species to routinely perform aerial dives into the water to capture prey, diving to depths of up to $2 \mathrm{~m}^{4,7}$ In calm air, gliding typically occurs at airspeeds between 9 and 11 $\mathrm{m} / \mathrm{s}$ depending on atmospheric conditions, though speeds of up to $15 \mathrm{~m} / \mathrm{s}$ have been noted in some cases. ${ }^{3,7}$ Brown Pelicans are often seen gliding low over the ocean to take advantage of the ground effect, which simultaneously increases the lift and decreases the drag experienced by the bird, resulting in a decreased energy expenditure during flight. To further increase flight efficiency, Brown Pelicans may take advantage of rising air currents or thermals during soaring flight. ${ }^{3}$ Slotted primary feathers located at each wing tip may be manipulated in flight and are hypothesized to provide drag reduction and aerodynamic control based on flight conditions.

The slotted primary feather geometry of the Brown Pelican makes this large gliding avian an ideal candidate for this investigation. While the model creation and analysis techniques outlined in this study may be utilized for any avian wing tip geometry, the Brown Pelican has been chosen here to provide a foundation for future studies investigating the aerodynamics of a Brown Pelican wing while in ground effect. 
Forces and moments imparted on a body traveling through a fluid are due to two primary factors: the distribution of pressure over the body surface and the distribution of shear stress over the body surface. While various aerodynamic forces may result from these distributions, Nature's ability to influence a body traveling through a fluid is restricted to these two effects alone. ${ }^{8}$ Two key forces generated when a body moves through the air, be it an aircraft or a bird, are lift and drag. A more in-depth discussion follows.

\subsection{Lift}

Lift is the resultant aerodynamic force acting on a body normal to its velocity vector and is typically produced by specialized structures such as wings. While the force generated by lift is typically used to counteract the weight of the body to enable steady flight, this is not necessarily always the case. In the case of subsonic flight below Mach 0.3, the generation of lift can be explained in a relatively straightforward manner by employing a simplified form of the Bernoulli equation:

$$
P+\frac{1}{2} \rho V^{2}=\text { const }
$$

where $\mathrm{P}$ is the local static pressure, $\rho$ is the density of the fluid and $\mathrm{V}$ is the local velocity of the fluid. In a steady and inviscid flow, the above equation is equal to a certain constant (often referred to as the total pressure) for any point along a streamline in that flow. This leads to the important conclusion that when the static pressure of a flow increases, the velocity of the flow decreases and vice versa. A lift-generating wing, when properly designed, will cause the air traveling over it to flow at a higher velocity than the air traveling beneath it. By referencing Equation 1, it can be inferred that this change in velocity will result in a region of relatively low pressure above the wing and a region of relatively high pressure below the wing. It is this pressure differential that imparts the force of lift to a wing. The strength of the regions of low and high pressure (and hence the 
amount of lift) generated by the wing can be influenced to some degree by modifying several key geometric parameters of the cross section of the wing or by change the angle between the airfoil chord and the relative wind. These parameters will be discussed in greater detail in Section 3.3..$^{8,11}$

\subsection{Drag}

The resistive force experienced by a body moving through the air is a function of three primary drag effects: parasitic drag, induced (or "lift-dependent") drag and wave drag. In the realm of aerodynamics, wave drag is related to the formation of shock waves in a flow and typically only a factor at relatively high speeds where the effects of flow compressibility become non-trivial. Consequently, it will be disregarded for this discussion of low speed aerodynamics. The term "parasitic" drag covers several types of drag effects, such as form drag, skin friction drag and interference drag. Drag related to these effects is typically a function the geometry of the body in question, including its overall shape and size. At lower speeds, however, drag effects are primarily a function of induced drag. ${ }^{8}$

As a byproduct of the generation of lift, a finite wing will produce regions of relatively low and high pressure above and below the wing, respectively. This net imbalance of pressure causes the high pressure air below the wing to "leak" out around the wing tip into the region of low pressure air. This introduces a spanwise component to the flow over the wing, with airflow above the wing moving towards the wing root and airflow below the wing moving away from the wing root. The curl of air over the wing tip and spanwise flow components contribute to the creation of a region of recirculating flow, or vortex, at the wing tip. These vortices introduce areas of relatively low pressure downstream of the wing, leading to a net pressure force in the downstream direction as a result of the relatively higher pressure immediately upstream of the wing. This may be essentially thought of as a drag force. ${ }^{8}$ 
The wing tip vortices created by a finite wing not only increase the amount of drag experienced by the wing, but also force the air in the vicinity of the wing in a downwards direction. This downwash reduces the effective angle of attack $(\alpha)$ that the wing experiences, thereby reducing the total lift generated by the wing. ${ }^{8}$

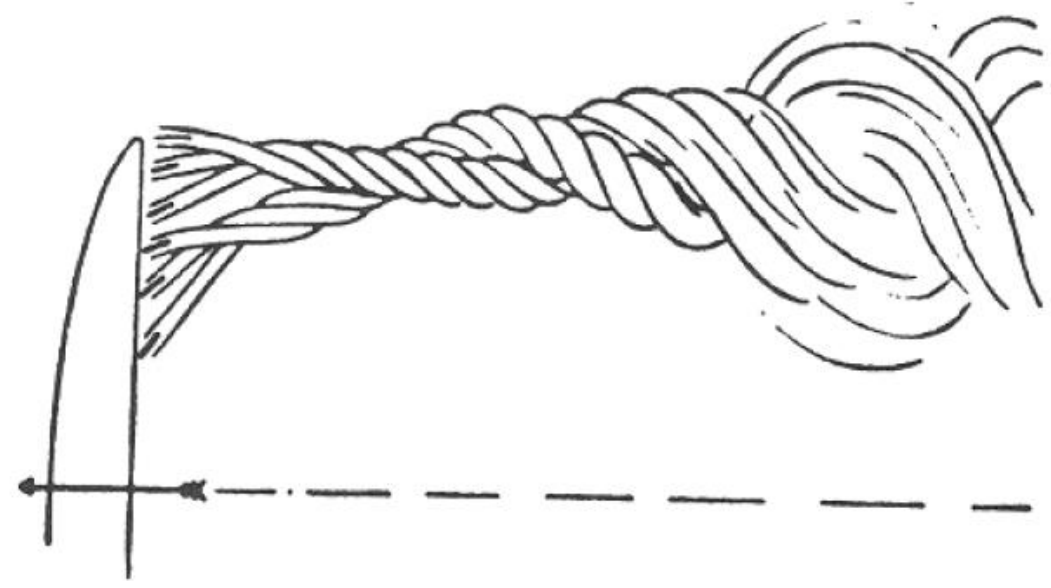

Figure 2. Visualization of wing tip vortices created by a finite wing. ${ }^{9}$

Clearly there is a strong incentive for the aerodynamicist to reduce the induced drag generated by a wing from an efficiency standpoint. The generation of induced drag by the tip of a finite wing can be addressed in several ways. One method is to increase the aspect ratio, or the span of the wing squared normalized by the surface area of the wing. Another is by designing the wing to have an elliptic lift distribution. While a thorough discussion of these concepts and their effect of the efficiency of a wing is beyond the scope of this work, additional information may be found in Reference 8. In situations where these options are not available, a third option exists: the additions of winglets. When properly designed, winglets reduce the size and strength of trailing vortices. By limiting the formation of vortex structures downstream of a wing through the use of winglets, the total drag and downwash experienced by a wing can be significantly reduced. 


\subsection{Airfoil Nomenclature}

The aerodynamic properties of a lifting surface, be it an aircraft wing, helicopter blade, bird wing, etc., are strongly influenced by the shape of the wing section, often referred to as an airfoil. For subcritical Mach number flows where the chordwise flow component is much larger than the spanwise component, the aerodynamic properties of a wing's airfoil sections may be used to approximate the aerodynamic properties of the wing as a whole. Several key geometric properties, such as the chord, mean camber line, camber, thickness and leading edge radius can be used to determine the exact geometry of an airfoil section. This nomenclature was developed in the early 1930's by the National Advisory Committee for Aeronautics (NACA), the predecessor of the National Aeronautics and Space Administration (NASA), during extensive testing of systematically constructed airfoils. Figure 3 presents an overview of these terms. ${ }^{8,11}$

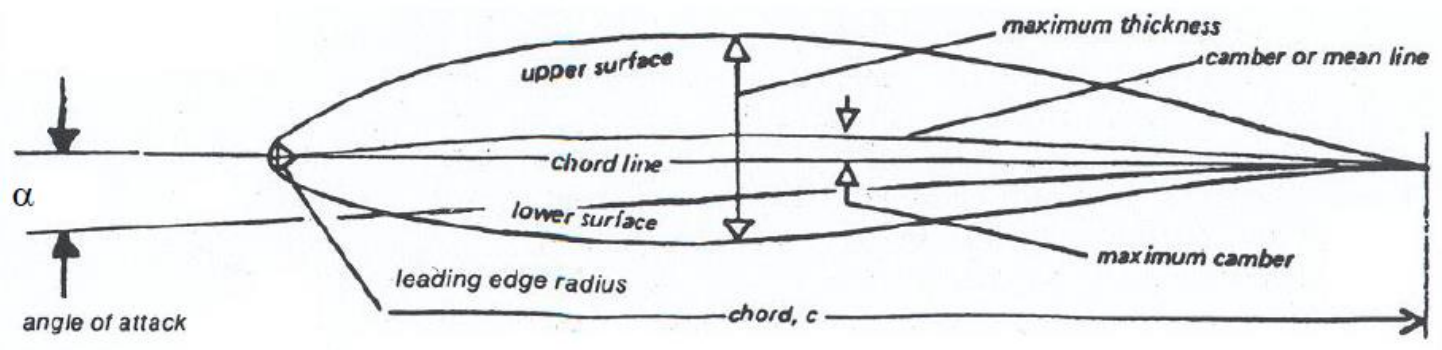

Figure 3. Common properties of airfoil geometry. ${ }^{10}$

The chord of an airfoil is determined by connecting the leading and trailing edges using a straight line. The mean camber line essentially acts as a mean of the upper and lower surfaces of the airfoil and is located half way between each surface. The camber is defined as the maximum distance between the chord and the mean camber line when measured perpendicular to the chord. Similarly, the thickness is the maximum distance from the upper and lower surfaces when measured perpendicular to the chord. Also of note is the angle of attack $(\alpha)$, which defines the angle between the chord line of the airfoil and the vector of the incoming air flow. ${ }^{8,11}$ 
Early studies inspired by the wings of birds focused on the aerodynamics of extremely thin wings (the term "thin" is here used to refer to airfoils with a maximum thickness to chord length ratio of 0.12 or less). This is evidenced by the thin airfoil sections used by many early aircraft designs, up to and including many World War I aircraft. Subsequent research indicated that lift generation could be enhanced by increasing the thickness and camber of an airfoil, as well as the leading edge radius. However, increasing these properties beyond certain limits may cause a significant increase in drag, leading to severely degraded performance of the airfoil. These geometric parameters also control, in part, the transition between laminar and turbulent boundary layers.

An exhaustive compendium of performance data for a multitude of NACA airfoils may be found in Reference 11. An in-depth discussion of the history and mathematics behind airfoil sections may be found in Reference 8 .

\subsection{The Modern Winglet}

While the first examples of semi-functional aircraft winglets started to appear at the turn of the $20^{\text {th }}$ century, the first winglet in the modern sense of the word appeared in the 1970's with the work of Richard Whitcomb. Whitcomb, who is also known for his work involving the area rule and supercritical airfoils at NASA's (then NACA) Langley Research Center, was inspired by the wing tip feathers of large gliding and soaring birds. Wind tunnel tests and subsequent flight testing on a retrofitted $\mathrm{KC}-135$ indicated a $20 \%$ decrease in induced drag and a $7 \%$ increase in the lift-todrag ratio, a significant accomplishment in the world of aeronautics. With the cost of aviation fuel climbing ever higher throughout the 1970's, many aircraft manufacturers began to implement winglets on a wide array of aircraft, ranging from large commercial jets to small propeller-driven aircraft. $^{2}$ 
Modern winglets can vary significantly in size and shape depending on the needs of the specific aircraft. Regardless, the goal of each winglet remains the same: to reduce the induced drag generated by the wing and increase the efficiency of the aircraft. This is generally accomplished by modifying the effective aspect ratio without significantly increasing the span of the wing. Winglets offer improved efficiency without the need to lengthen the wing, which would require additional reinforcement of the wing structure and increase the total weight of the aircraft, further increasing the fuel burn. A collection of several modern winglet designs are presented below.
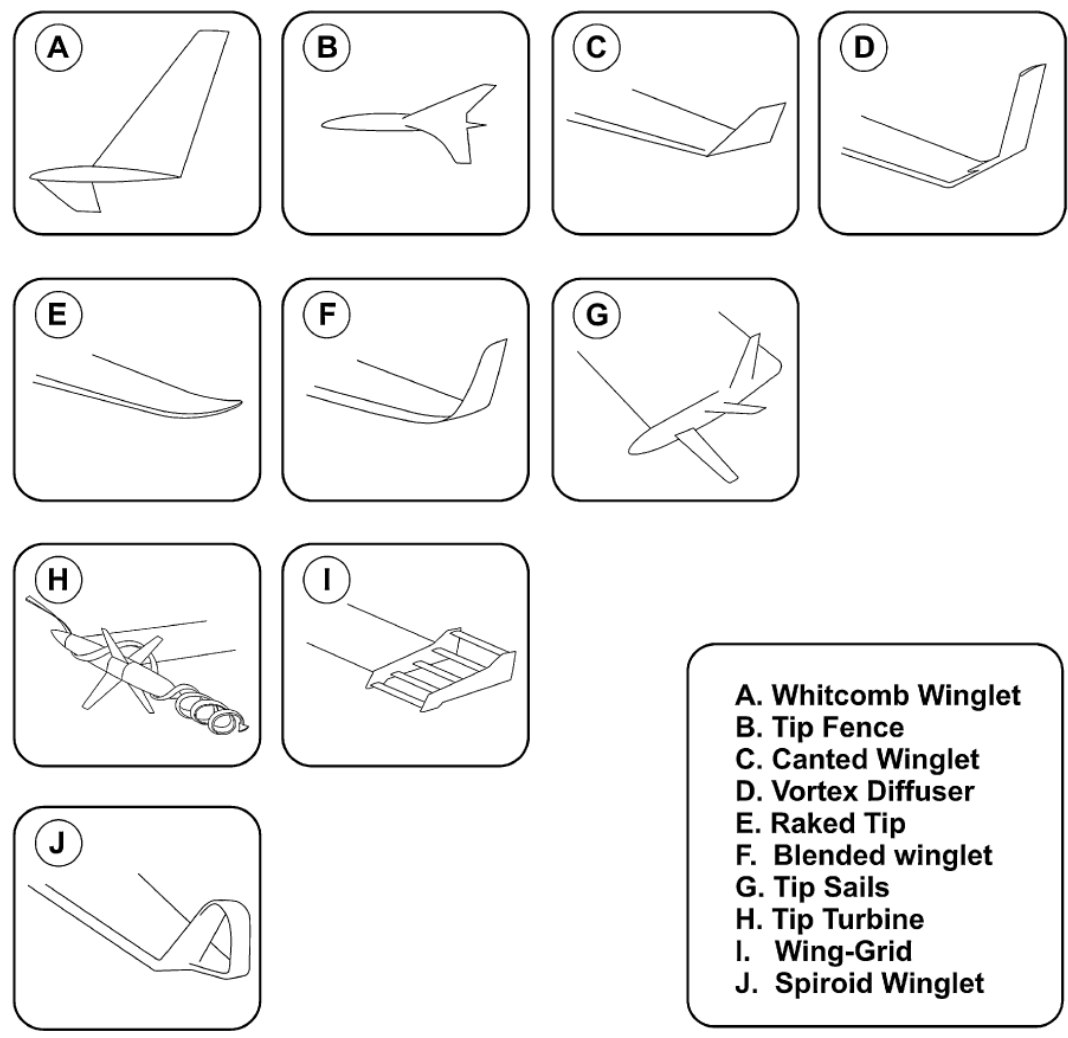
A. Whitcomb Winglet
B. Tip Fence
C. Canted Winglet
D. Vortex Diffuser
E. Raked Tip
F. Blended winglet
G. Tip Sails
H. Tip Turbine
I. Wing-Grid
J. Spiroid Winglet

Figure 4. A sample of modern winglet designs. ${ }^{1}$

Once the effectiveness of the basic winglet geometry had been confirmed by NACA, researchers began investigating more radical geometries in an effort to improve performance even further. This resulted in a number of unconventional designs, such as the wing-tip turbine and 
spiroid winglet. However, several designs bear a striking (albeit simplified) resemblance to the primary feather configuration of large gliding birds, such as the wing-tip sail, wing-grid and the modern split scimitar winglet (not pictured in Figure 4).

\subsubsection{Biomimetic Testing}

While Whitcomb's inspiration for the modern winglet was indeed based on the slotted primary feathers of large gliding birds, the complex biological shape of this geometry has hampered efforts at artificial replication. As a result, related studies have typically used highly simplified test models. These approximations, while retaining some amount of resemblance to their original biological counterpart, often simplify avian wing tip geometry to such a degree that it is no longer clear how comparable the two geometries truly are in terms of aerodynamic performance. Many studies present unusual and contradictory results, leading to confusion about the efficacy of various winglet designs.

As a case in point, a study performed by Coiro et al. compared two simplified slotted primary models, one with three cascading winglets and one with five cascading winglets, to a base wing configuration. The models were compared in terms of Oswald factor, an efficiency factor representing the change in drag with lift. While the five winglet geometry was found to decrease the induced drag experienced by the wing when compared to the base wing, the three winglet geometry was instead found to increase the drag. Furthermore, a comparison between the five winglet geometry and a standard, classical winglet suggested that the classical winglet was more effective at reducing induced drag. While the authors noted that the junction between each winglet and the wing tip on both cascading winglet models was poorly optimized (a common issue among studies using simplified geometries), this does not fully explain the unexpected and perplexing results. $^{12}$ 
Studies investigating a larger number of configurations or employing optimized positioning of each winglet appear to give more reliable data. A reduction in reduced drag has clearly been achieved along with increased efficiency and performance in a number of cases, primarily at lower speeds (approximately $50 \mathrm{~m} / \mathrm{s}$ or less) where the effects of induced drag are most apparent. A pair of studies by Catalano et al. using cascading winglets with variable positioning and angle control revealed that, of the 55 tested winglet configurations, only 6 configurations provided an increase in efficiency and performance, whereas the remaining configurations provided no benefit at all or even increased the drag experienced by the wing. From these studies, it appears that while a cascading winglet geometry may indeed provide benefits in terms of efficiency and performance, the positioning and geometric characteristics of each winglet must be optimized for each application and that a "one size fits all" approach likely does not exist. ${ }^{13,14}$

Approaches using numerical optimization techniques have also yielded some degree of success. During a study of wing tip geometries on the U.S. Marine Corps Dragon Eye UAV using a panel method code that approximated each winglet as a flat plate, Shelton et al. discovered that the addition of distributed winglets did in fact increase the range and endurance of the UAV. However, it was noted that this depended strongly on the length and positioning of each winglet, as well as the flight conditions. ${ }^{16}$
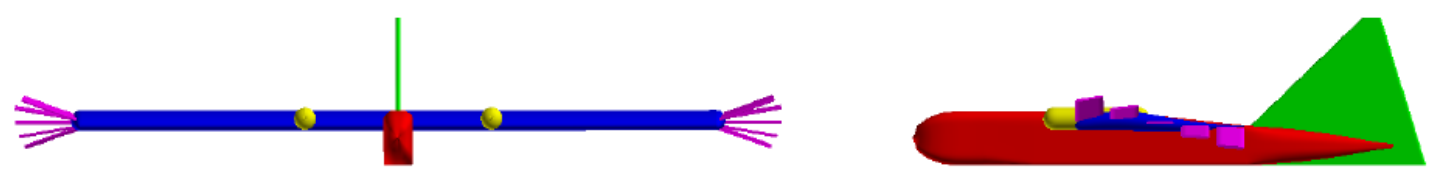

Figure 5. Dragon Eye UAV with cascading winglet geometry investigated by Shelton et al. ${ }^{16}$

A number of more recent studies have focused on a relatively novel geometry: the spiroid winglet. While still inspired by slotted primary feathers, spiroid winglets simplify the geometry by 
connecting two conventional vertical winglets with a horizontal section (see J in Figure 4). Other approaches simplify this geometry even further by constructing a fully closed ribbon-like loop at the wing tip. Preliminary investigations have been promising, showing an induced drag reduction ranging from $28 \%$ to $75 \%$ depending on flight conditions while simultaneously increasing total lift generated by the wing. Despite potential benefits, spiroid winglets are not without their shortcomings as well. The overall size of a spiroid winglet would lead to a marked increase in parasitic drag as well as an increased aircraft weight, due to both the increased weight of the winglet and the necessary reinforcement of the wing required to support this extra weight. ${ }^{1}$

Additional studies have proposed even more radical ideas, such as morphing winglets with a variable span and cant angle. Morphing winglets could adapt to various flight conditions and could potentially offer an additional 2-3\% improvement in aircraft fuel efficiency. Other studies of adaptive winglets able to modify their angle of attack, dihedral and sweep in flight have revealed that, in addition to potential gains in performance and efficiency, adaptive winglets could also be used for yaw and roll control, reducing or even eliminating the need for a conventional vertical stabilizer. While it would appear that potential gains in efficiency continue to point to the wingwarping, rudderless avian flight configuration, care must be taken when investigating morphing technologies so as not to add an excessive amount of weight to aircraft designs. ${ }^{15,16}$

It can be seen then that there is some degree of finesse involved in the design of biomimetic winglets. The success or failure of a cascading winglet design appears to hinge on a number of factors including the size and weight, as well as the splay angle and angle of attack of each winglet. Ultimately, ensuring the success of a winglet design revolves around a critical balance between the addition of weight to an aircraft and the reduction of drag over a wide range of flight conditions. 


\section{AVIAN MORPHOLOGY}

\subsection{Wing Feathers}

The avian feather undoubtedly plays a crucial role in enabling flight. While the geometry of specific feathers can vary significantly depending on location and purpose, all feathers are primarily composed of the protein beta-keratin. In addition to their role in flight, feathers can also provide other benefits, such as insulation, camouflage, weatherproofing and fairing of the body structure. ${ }^{23}$ For the sake of simplicity, only the flight feathers located on the wing will be discussed here. The positioning of these feather groups with relation to the wing skeleton of a Brown Pelican may be found in Figure $7 .^{22}$

Wing feathers (occasionally referred to as remiges) generally include the primary and secondary feather groups. These are characterized by the asymmetric positioning of the feather shaft, resulting in a noticeably smaller leading edge, as well as their attachment to the skeletal structure. Direct attachment to the skeletal structure of the wing allows for the transmission of aerodynamic loads from the vane of each feather to the bird's skeleton via the feather shaft. The primary feathers are typically the longest of all avian feathers and are located at the wing tip, attaching directly to the phalanges and carpometacarpus. As they are anchored to the "hand" of the bird, they may be spread or rotated independently with some degree of control. Primaries are thought to serve a number of purposes beyond the generation of lift depending on the species in question. These can range from drag reduction to aerodynamic noise reduction and even some degree of aerodynamic control. ${ }^{23}$

Mature Brown Pelicans typically have 10 primary feathers at the tip of each wing. Figure 6, generated by the United States Fish and Wildlife Service, presents the overall size and shape of each of these primary feathers. Of particular note are the distinctly slimmer and elongated feathers present near the wing's leading edge, as well as the asymmetric positioning of the shaft in each feather. 


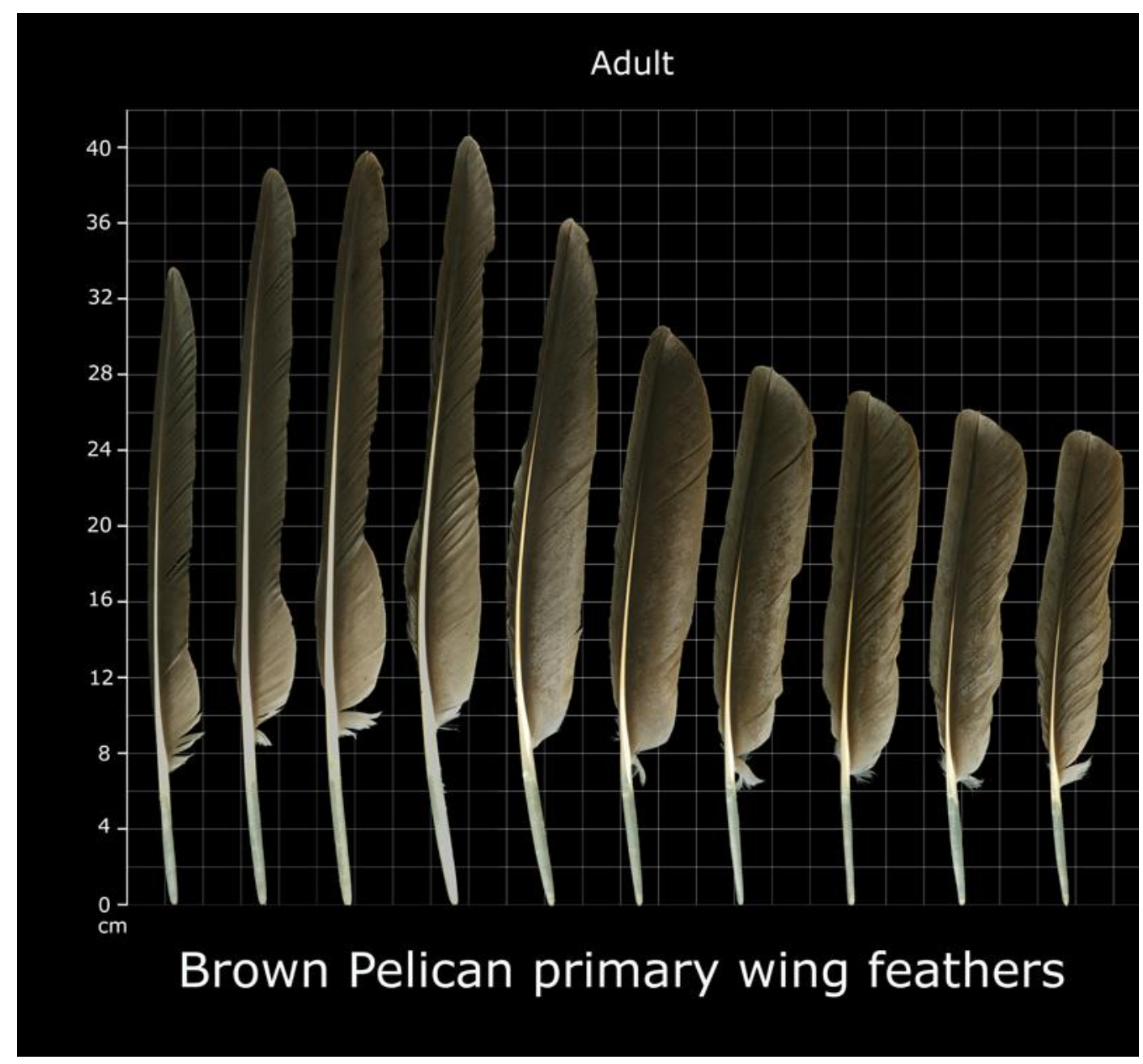

Figure 6. Morphology of the primary feathers of a Brown Pelican in order from the leading edge (left) to the trailing edge (right) of the right wing. ${ }^{24}$

Secondary feathers are typically somewhat shorter and more broad than the primary feathers and help to create the airfoil section of a bird's wing. These feathers attach to the ulna and are unable to be manipulated without movement of the entire bone. Tertial feathers, which are generally not considered to be true flight feathers, attach to the skin of the bird only and exist to protect the folded wing and blend the wing and body shapes during flight. A detailed discussion regarding the pelicaniform skeletal structure and feather attachment may be found in Reference 22 . 


\subsection{Skeletal and Muscular Systems}

The avian skeletal system is composed of a collection of extremely lightweight bones, many of which are hollow. While the rear limbs of modern avian species have been retained as legs, the forward limbs have evolved into specialized wing structures. The phalanges retain some degree of movement allowing articulation of the primary feathers, though the majority of the metacarpal bones have been fused together, limiting the overall wrist movement. Articulation of the wing bones accomplished by a collection of muscles in each wing. While the powerful muscles enabling flight are primarily located in the chest and shoulder areas, each wing houses some two dozen individual muscles tasked with articulating the wing into a wide range of shapes. Despite the large number of wing muscles, the area between the shoulder and wrist joints is occupied by a collection of tendons only, which pushes the maximum thickness of the wing further aft. An overview of the skeletal structure and flight feather groups of a Brown Pelican wing is presented in Figure 7..$^{21,22}$

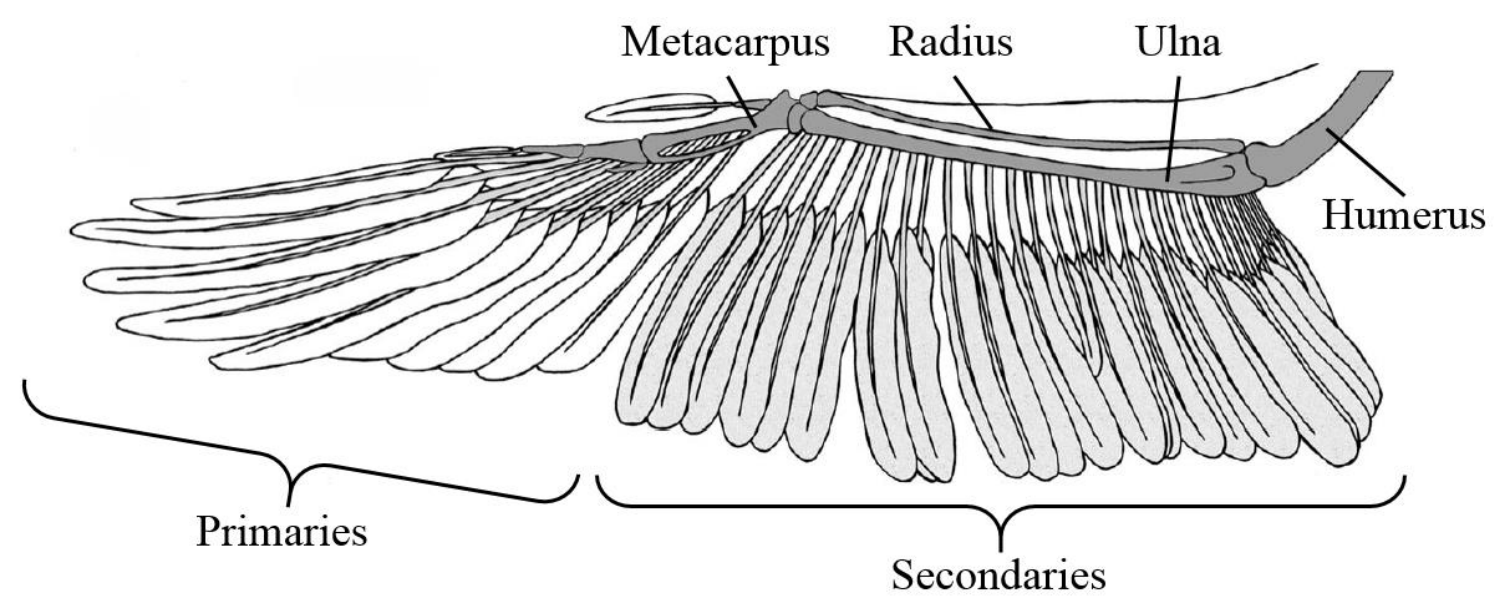

Figure 7. Overview of the Brown Pelican wing structure presented by Simons et al. Labels have been added for clarity. ${ }^{22}$ 


\subsubsection{Metabolic Cost}

A prime consideration when discussing the aerodynamics of avian species is the metabolic cost incurred during flight. While this topic often falls under the purview of biologists rather than aerodynamicists, the results are nonetheless crucial to the understanding of flight feathers and their uses. A study conducted by Schnell et al. utilized Doppler radar techniques to investigate which of the following quantities the wings of different species of gulls, terns and skimmers were minimizing during flight: the metabolic rate required for flight (minimum power), the metabolic cost of transport over the ground or the metabolic cost of transport through the air. It was discovered that, while the flight mode was highly dependent on environmental and ecological factors, the birds generally chose a flight mode to minimize the metabolic cost over the ground. ${ }^{17}$ However, Hedenstrom et al. note that these types of studies can be a tricky business as it is not necessarily known to the investigator what factors the bird is aiming to optimize in a given ecological situation regardless of experimental technique, making it difficult to apply the appropriate mathematical optimization theory. A significant amount of additional data regarding the power required at various flight speeds during multiple flight segments (gliding, foraging, migrating, etc.) will need to be gathered for each species individually before a quantitative determination can be made regarding avian efficiency in certain ecological situations. ${ }^{18}$

\subsection{Experimental Techniques}

Conducting aerodynamic studies using live birds can present a multitude of difficulties. In addition to the potential moral and ethical quandaries related to placing live animals in a wind tunnel or other controlled testing environment, these animals would also need to be trained to perform in a way that allows for the consistent collection of useful data. Observation of birds in the wild is certainly a possibility, though this introduces enough variability in the experimental process to make any sort of detailed aerodynamic study near impossible. Nevertheless, numerous studies 
to characterize avian flight using live birds have been performed, such as the series of studies performed by Spedding et al.

In these studies, various birds were observed during flight in a large flight cage or down a long corridor. Vortices created by the bird's wing tip were visualized using a series of cameras, flashbulbs and soap bubbles filled with helium suspended in the flight path. However, a common theme among these types of studies is the difficulty in achieving consistent and reasonable results. While relevant, these studies were carried out using relatively small birds (kestrels, jackdaws, pigeons, etc.) whose wings are more optimized for speed rather than gliding or soaring. ${ }^{19,20}$

In other cases, researchers have chosen to take a more direct approach by placing specimens in a small-scale wind tunnel with an articulating test section. This is useful as the test section can be rotated in such a way as to produce ideal gliding conditions within the test section indefinitely, allowing much more data to be captured between wing beats. In a study of the Harris' hawk (Parabuteo unicinctus) using this methodology, Tucker compared two primary feather configurations: one with wholly intact primary feathers and one with clipped primary feathers. Clipping of the primary feathers was found to create a pronounced difference in the amount of induced drag experienced by the hawk, with the slotted primary feather configuration creating up to $50 \%$ less induced drag. ${ }^{25}$ Studies using a similar methodology found that a gliding jackdaw (Corvus monedula) spent a significant amount of time gliding in a configuration that maximized the ratio of lift to drag, or $\mathrm{L} / \mathrm{D}$ ratio. This strongly suggests that birds do in fact take an active role in minimizing the metabolic cost during flight as the value of the $\mathrm{L} / \mathrm{D}$ ratio plays a large role in the efficiency of a wing. ${ }^{26}$ 
The body of work for this research project was separated into two primary components: the establishment of a process allowing the creation of biologically accurate models suitable for aerodynamic testing and the implementation of this process to characterize the flow field downstream of an example model. An overview of the project workflow is presented in Figure 8.

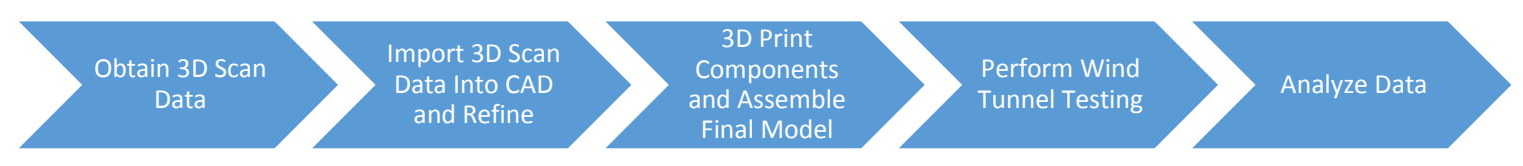

Figure 8. Project workflow overview.

\subsection{Biological References}

Examples of both a Brown Pelican primary feather and a taxidermized wing were obtained to serve as references during the $\mathrm{CAD}$ model creation process. The example primary feather was loaned by Dr. Krista Fahy and the Santa Barbara Museum of Natural History in Santa Barbara, California. This particular feather was the ninth primary feather (P-9, second feather from the leading edge) on the left wing and was obtained from an existing specimen on cold storage at the time of the loan. The overall length of the feather when pressed flat was approximately $40 \mathrm{~cm}$. 


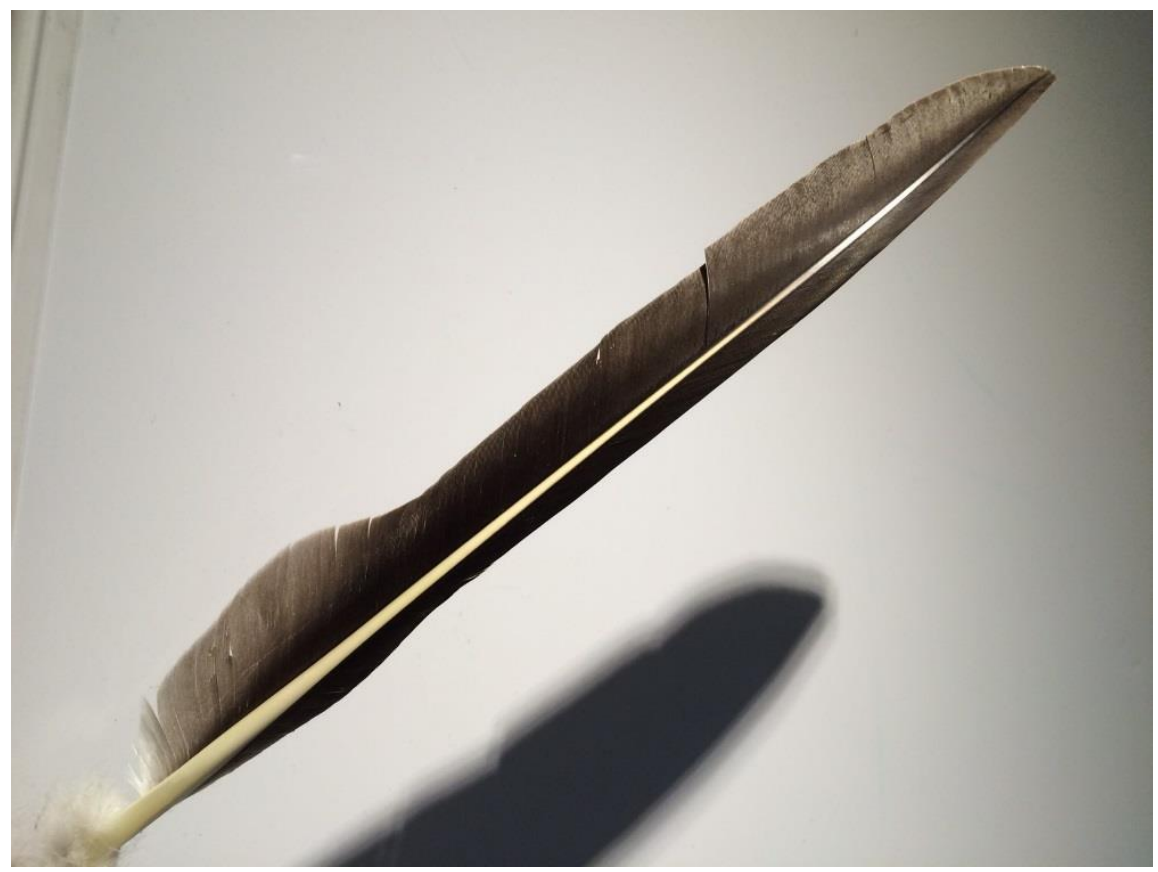

Figure 9. Brown Pelican primary feather loaned by the Santa Barbara Museum of Natural History.

A preserved example of a left wing in flight configuration was provided by the Los Angeles Natural History Museum. The wing had a span of $0.82 \mathrm{~m}$ and an average width (leading edge to trailing edge) of approximately $0.25 \mathrm{~m}$. The wing was 3D scanned on location during a day trip, along with examples of both a California Condor (Gymnogyps californianus) wing and primary feather. While not addressed directly in this study, the 3D scans of the condor wing and feather have been retained for use in future projects. 


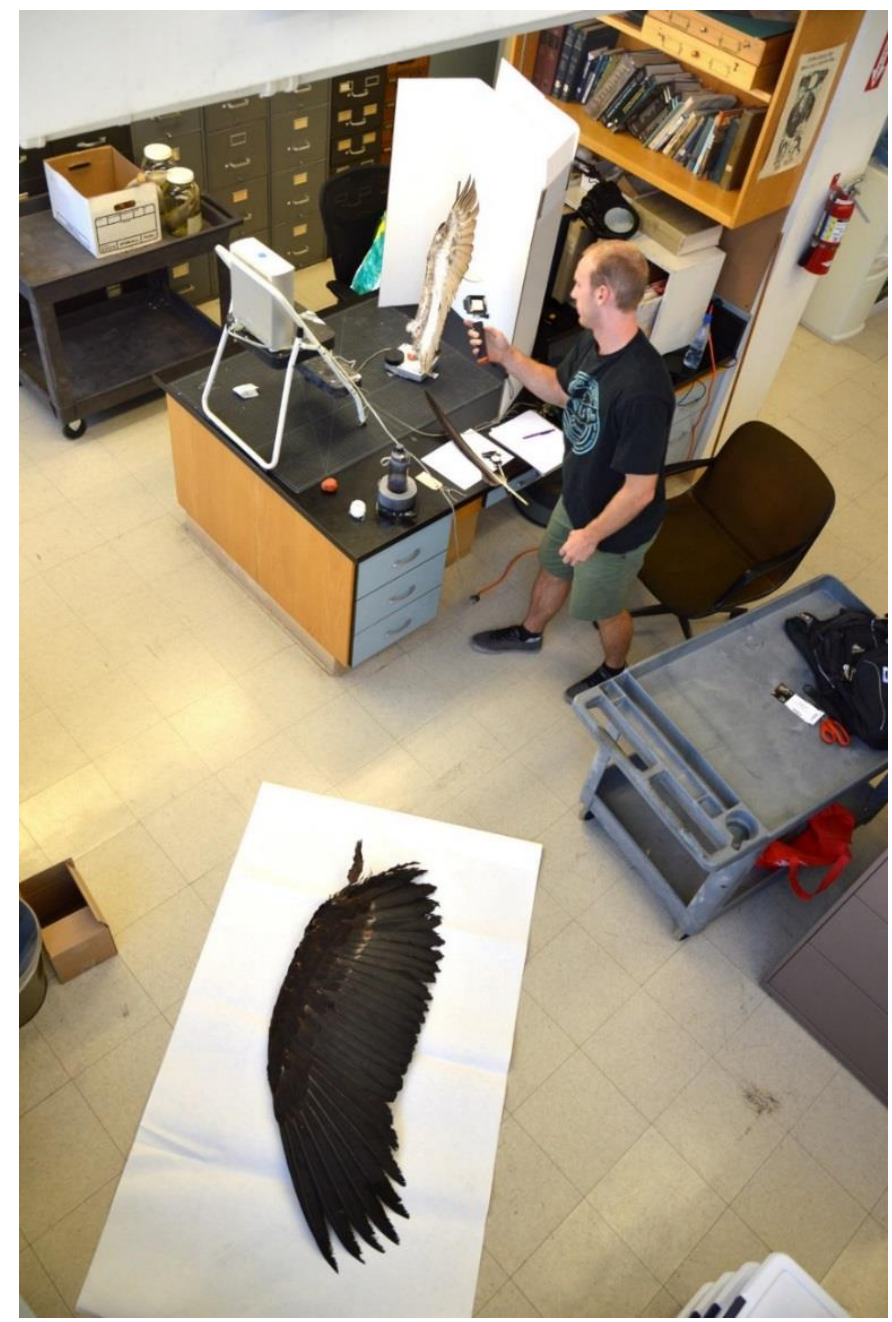

Figure 10. 3D scanning of a Brown Pelican wing at the Los Angeles Natural History Museum.

Visible on the floor is the preserved wing of a California Condor.

Video footage provided by the BBC of Brown Pelicans in flight was reviewed to determine the general shape and positioning of the primary feathers while in a flight configuration. Still images depicting the pelicans from both side views and head-on views were then imported into SolidWorks 2016, a common computer-aided design (CAD) program created by Dassault Systemes. Spline sketches were used to capture the general size and curvature of each primary feather as accurately as possible. The resulting sketch data was then utilized to create a series of five surfaces representing each of the primary feathers. These surfaces were later used to 
approximate the size and curvature of the primary feathers of the final CAD model. While the amount of splay may vary between individual birds based on a wide variety of conditions, the geometry captured here represents the most common splay configuration noted in the video footage during gliding flight.

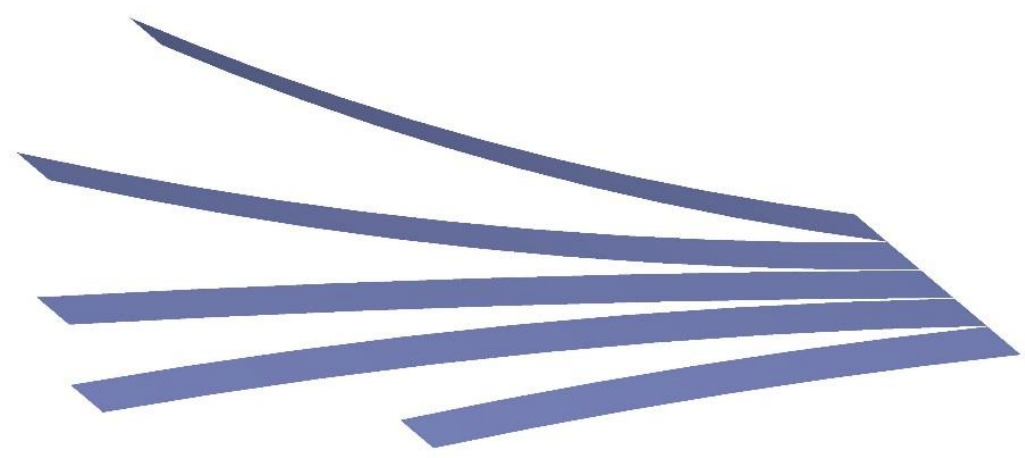

Figure 11. CAD surfaces used to determine the general size and curvature of the primary feathers on the final model.

\subsection{D Scanning}

3D scanning was performed using a NextEngine Ultra HD 3D Laser Scanner. The main scanner unit $(22.4 \mathrm{~cm} \times 9.1 \mathrm{~cm} \times 27.7 \mathrm{~cm})$ houses a series of laser scanners and optical cameras, as well as a floodlight to illuminate the object to be scanned. A small turntable is also included, which can be controlled manually by the user or by the scanner during the automated scanning process. A white $0.9 \mathrm{~m} \times 1.2 \mathrm{~m}$ tri-fold poster board was placed behind the turntable to minimize any interference from background objects during the scanning process.

The NextEngine scanner operates by converting laser scan data into a finite number of points in three-dimensional space. The points in this point cloud are then connected using triangular faces to form a polygonal mesh, which is then colored based on data captured by the optical 
cameras. The scanner is capable of processing up to 25 million discrete points during the mesh generation process with a maximum resolution of approximately $0.1 \mathrm{~mm}$. The scanner hardware is managed by NextEngine's ScanStudio software, which also doubles as platform for manipulating and merging scan files when necessary. ScanStudio is capable of exporting scan data in several common formats, such as STL, OBJ, XYZ and PLY. For the purposes of this project, the data was exported in both STL and XYZ formats.

A STL (stereolithography) file is essentially a direct export of the polygonal mesh generated by the ScanStudio software. The STL exports of both the primary feather and wing data can been seen in Figure 12. The XYZ format simply exports a list of the spatial coordinates of each point as a text file. These points may also be exported along with the corresponding normal vector of each point. While not used during this project, these normal vectors define the "outward" direction of the mesh and can be useful in defining the interior and exterior faces of the resulting mesh surface.

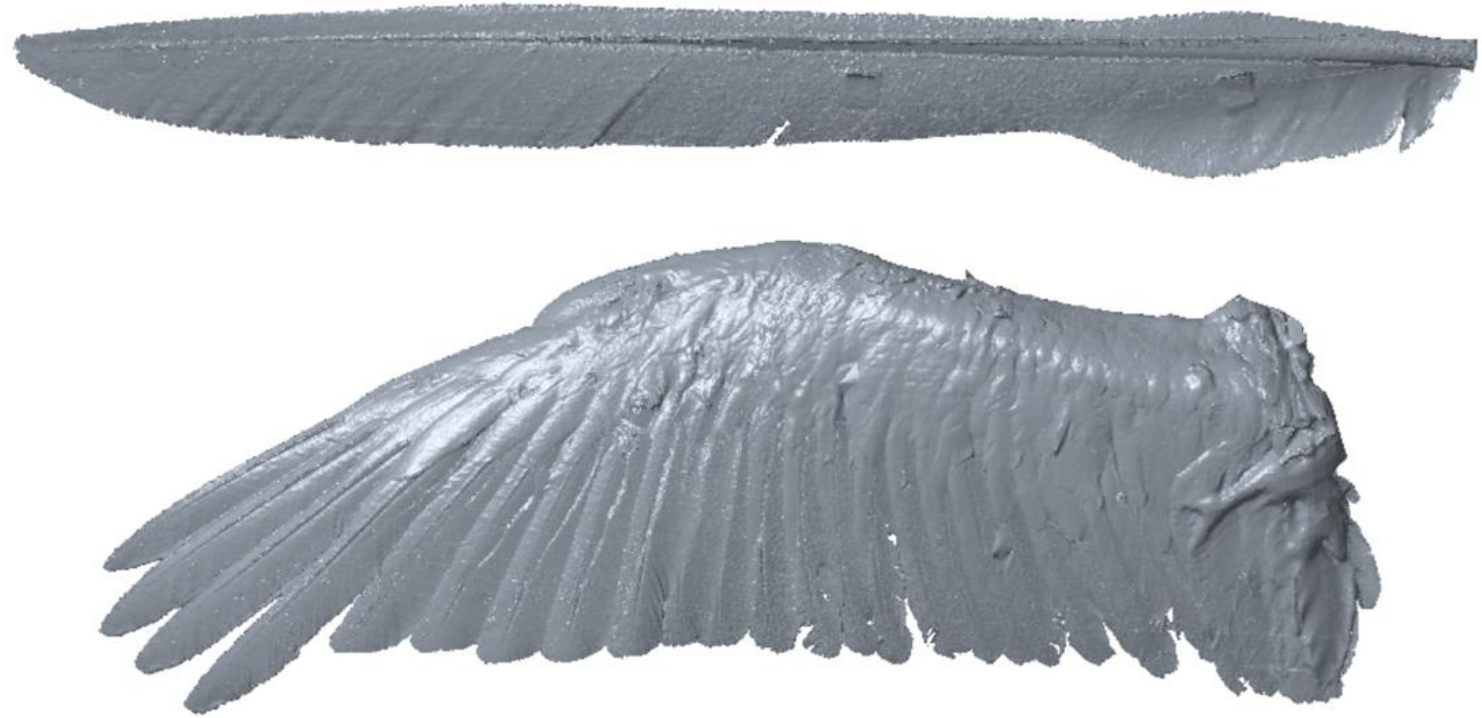

Figure 12. 3D scans of a Brown Pelican wing and primary feather generated by the NextEngine 3D scanner (not to scale). 


\subsection{Data Processing}

After completion of the scanning process, STL files generated by the ScanStudio software were imported into various CAD packages for additional refinement. However, it was discovered that the overall size and detail of each STL file caused considerable performance degradation when using either Creo Parametric or SolidWorks on the available laboratory computers (Dell Precision T7810 running 64-bit Windows 7 Professional, 2.40GHz Intel Xenon CPU and 32 GB RAM), typically resulting in software crashes. Due to continued difficulties related to the manipulation of the STL files in CAD space, it was determined that the numeric data would instead be manipulated directly using the STL geometry for reference.

To this end, a MATLAB script was developed to read the XYZ point cloud data generated by ScanStudio and split the data into a user-specified number of airfoil sections. This was accomplished by sampling a chosen number of points from the point cloud at calculated distances along the span of the feather or wing. Before running the script, each imported text file must be sorted along an axis using the geometric position of each point for reference. In this case, the feather and wing point clouds were sorted along their spans. Because the large number of data points exceeded the sorting capabilities of Microsoft Excel, sorting was carried out in Microsoft Access using a query-based approach. Future studies employing this process will likely streamline the sorting process by carrying out all necessary sorting directly in the MATLAB script before sectioning. After running the script using the sorted data, the resulting airfoil sections were exported as an OUT file. This extension was then manually modified to TXT to facilitate data import into various CAD programs.

The final sectioning configuration of the primary feather consisted of 15 individual airfoil sections each composed of 500 data points, whereas the final configuration for the wing consisted of 15 airfoil sections each composed of 1000 data points. While the MATLAB script was intended to be used to generate airfoil sections along a wing or feather span, it was constructed in such a way 
that any point cloud data can be sectioned along an axis provided that the data is properly sorted along that axis beforehand. The script was structured in this way such that it could be used for similar projects in the future. A copy of the script has been included in Appendix $\mathrm{C}$ for reference.

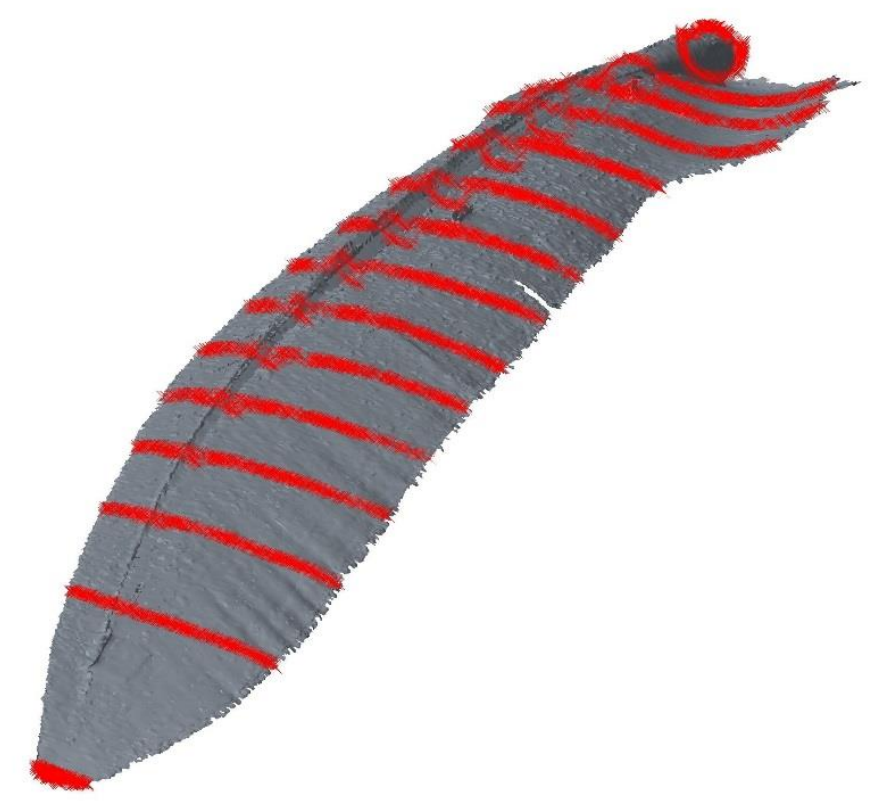

Figure 13. Highlighted airfoil sections generated by the MATLAB script overlaid on the primary feather STL geometry.

\subsection{CAD Modeling}

Creo Parametric (formerly known as Pro/ENGINEER), a leading CAD/CAM package created by the Parametric Technologies Corporation, was used extensively during the CAD model creation process. After importing the primary feather airfoil sections generated by the MATLAB script, complete airfoil sections were generated using two dimensional splines. The curve of the leading edge, trailing edge and feather tip were created using three-dimensional Datum Curve features. A single Boundary Blend feature was used to create the main body of the feather while a pair of smaller Boundary Blends were used to create the upper and lower surfaces of the feather 
tip. The resulting surfaces were then sewn together in a curvature continuous fashion and solidified to form the vane of the feather.

Using the original STL file for reference, two dimensional splines were then used to approximate the curve of the feather shaft from both a side and top view. These splines were then intersected to create the three-dimensional curve of the shaft. Five circular sections were placed along this Intersect Curve feature and their size and position was adjusted to mimic the shaft size and shape as accurately as possible. A Swept Blend feature was used to create the surface of the shaft, which was then solidified and joined with the feather vane. Figure 14 presents a comparison of the construction geometry and final solid geometry of the primary feather.

An identical process was then applied to the creation of the wing geometry. While the general airfoil shape created by the primary feathers was replicated as accurately as possible for the sake of completeness, much of this outer wing section was removed in subsequent operations to facilitate the addition of individual primary feathers. Figure 15 presents a comparison of the construction geometry and the final solid geometry of the wing body. 


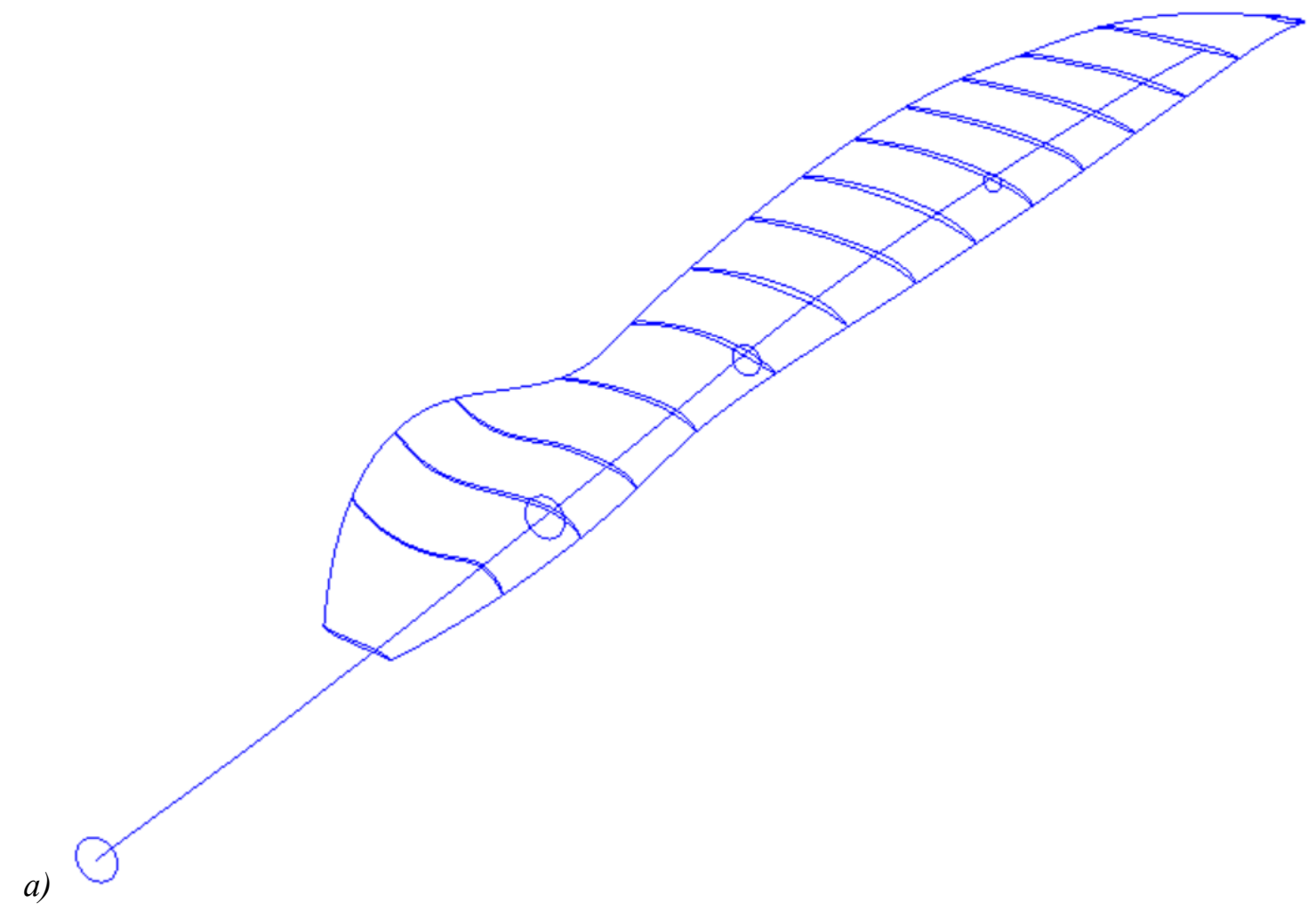

a)

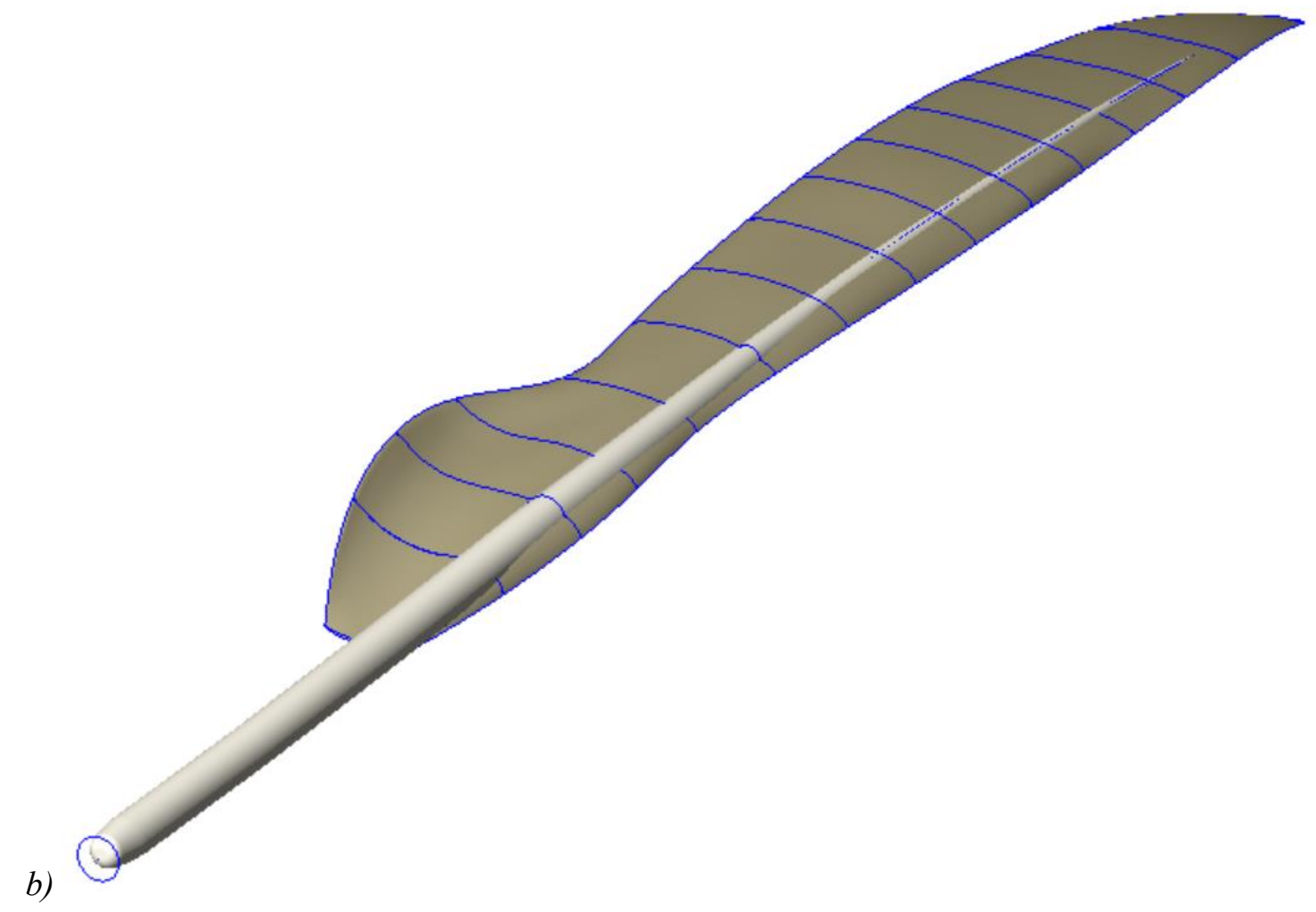

Figure 14. Comparison of the primary feather a) construction geometry and b) solid geometry. 

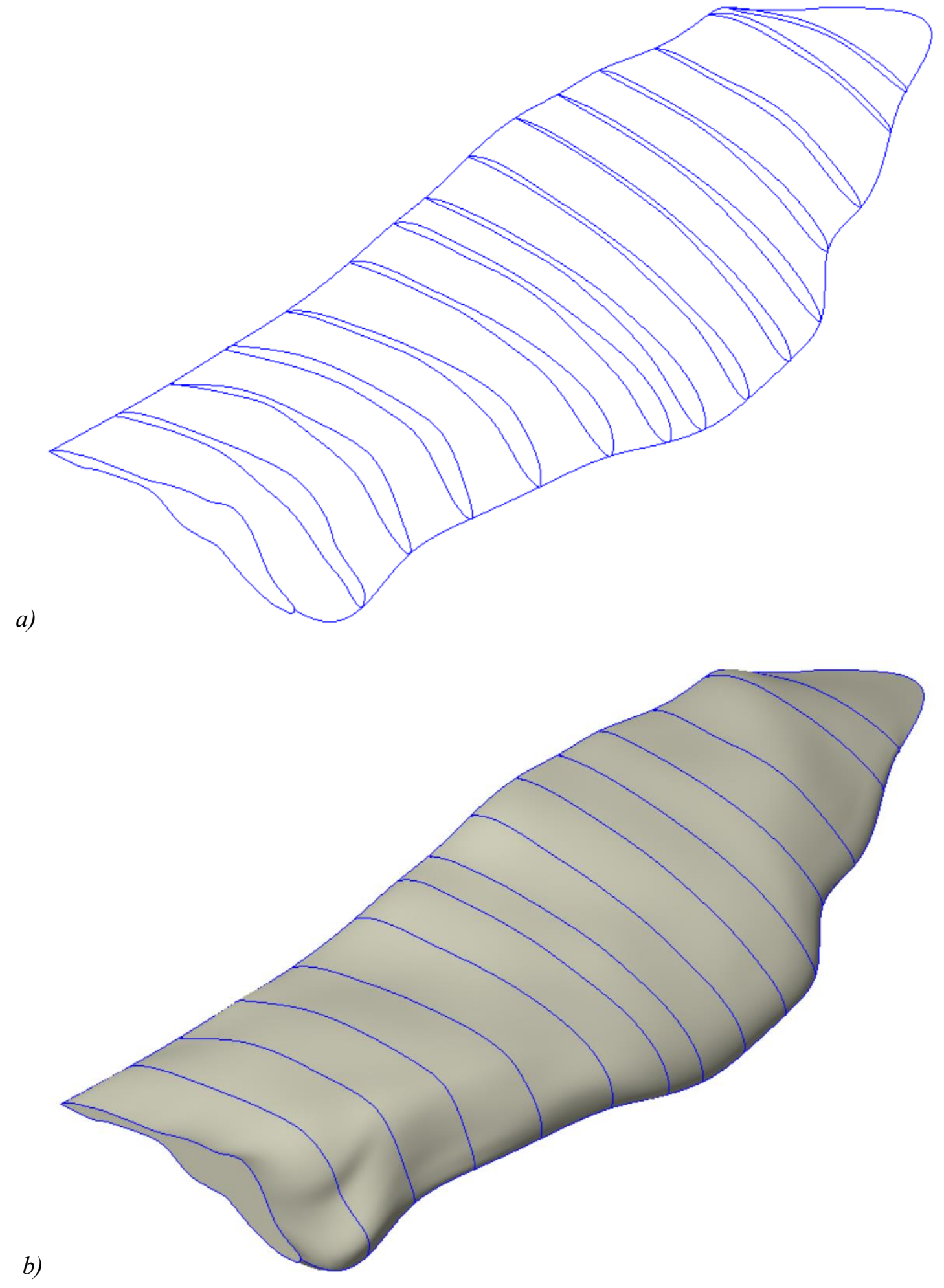

Figure 15. Comparison of the wing body a) construction geometry and b) solid geometry. 
After removing the highly contoured lower end of the feather to facilitate blending to the main wing body, copies of the primary feather model were then bent and placed at the end of the wing in accordance with the CAD surfaces generated from BBC footage detailed in Section 5.1. While some modern CAD packages contain a "Bend" function (or similar) that allows flexing of models around an axis, these functions are generally intended for use with relatively simple geometries. Experiments with the Bend command in SolidWorks generally resulted in the feather's complex surfaces collapsing and forming self-intersections. Feather bending was ultimately accomplished by manually moving airfoil sections vertically up or down in Creo Parametric until the curve of the feather matched that of the video study CAD surfaces. While tedious, this method provided a high degree of control over the final feather shape. Future projects will investigate the use of programs specifically designed for the manipulation of 3D objects and polygonal meshes such as Blender, an open-source computer graphics software developed by the Blender Foundation.

After properly positioning the five bent feathers in space with respect to the wing body, the final assembly was saved as a shrinkwrap file in Creo Parametric. Shrinkwraps essentially operate by sewing together all exterior faces in an assembly and solidifying them, resulting in a single solid model. At this stage, a 1:1 scale CAD model of a Brown Pelican wing with primary feathers in a gliding configuration was complete. However, due to concerns regarding the structural integrity and viability of $3 \mathrm{D}$ printing the extremely thin feather models, the decision was made to double the scale of the final test model in the fore/aft and up/down directions. No scaling was applied to the span of the wing. When attempting to apply this scaling in Creo Parametric, several of the complex surfaces and intersections between the feathers and wing body distorted enough to cause selfintersections and pierced faces. These surface discontinuities caused the model to revert to a collection of surfaces rather than a manifold solid. To combat this, the unscaled wing geometry was imported into SolidWorks as a non-parametric STEP model. Any undesirable geometry 
conditions were addressed and improved using the Import Diagnostics tool, resulting in a model that was both scalable and a manifold solid.

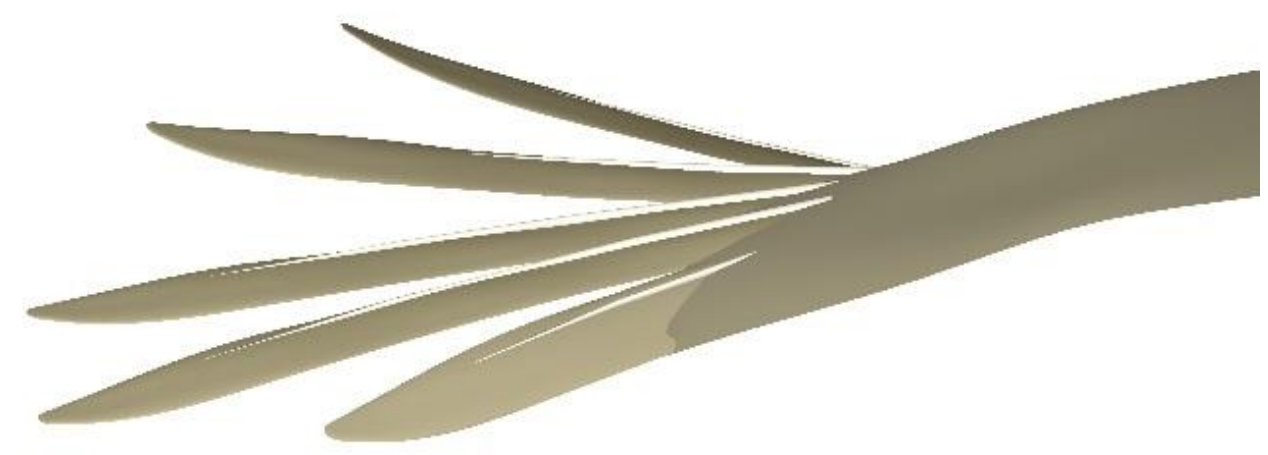

Figure 16. Detail of the final scaled wing geometry. View from the rear.

\subsection{Final Assembly}

For ease of model construction, the final assembly was split into two subassemblies each comprised of multiple designed parts and hardware. Assembly drawings detailing the top-level assembly and each subassembly can be found in Appendix B. Each assembly drawing contains a complete Bill of Materials of the components comprising the assembly. All assembly operations, necessary modeling (hardware, machined components, support structures, etc.) and drawing creation was carried out in SolidWorks.

\subsubsection{Body Assembly}

Because of the relatively large size of the scaled wing, the final body assembly was split into eleven discrete parts for 3D printing (see Figure 17). Several key considerations drove the selection of model split lines, as well as the overall size and shape of each component. Chief among these were the dimensions of the $3 \mathrm{D}$ printer print bed (to be discussed in greater detail in Chapter 
6), which limited the maximum size of components. Additional effort was made to reduce the number and severity of model overhangs, as these may cause significant issues during the printing process or even make printing impossible in extreme cases. Finally, the striations created during the $3 \mathrm{D}$ print process were chosen to be as parallel to the air flow over the wing as possible to minimize any cross-span flow interference. Holes and additional features were then added to the resulting parts to accept hardware necessary for the assembly and mounting of the components. A fairing structure was added to the wing root to enclose the necessary mounting hardware and prevent excessive disruption to the air flow. While open during assembly and disassembly, this area was covered with aluminum tape during testing to provide a smooth and continuous surface. A large $1.6 \mathrm{~mm}$ thick sheet metal splitter plate was mounted at the wing root to limit undesirable flow effects related to the boundary layer of the wind tunnel wall and the mounting interface.

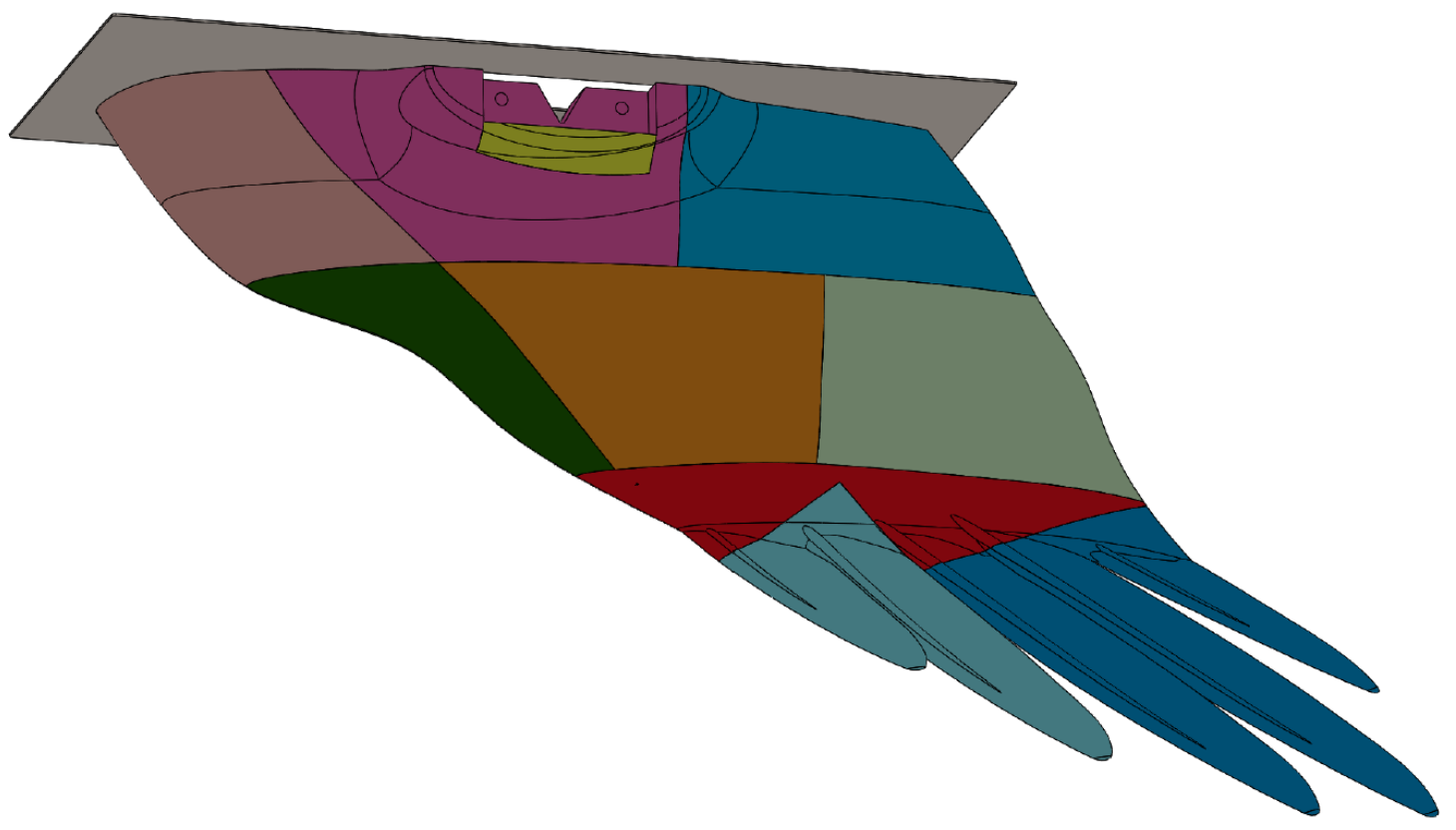

Figure 17. Wing body assembly overview in SolidWorks. 


\subsubsection{Mounting Assembly}

A mounting assembly was designed to mount the test model to the wind tunnel linear drive.

An overview of the mounting assembly is presented in Figure 18.

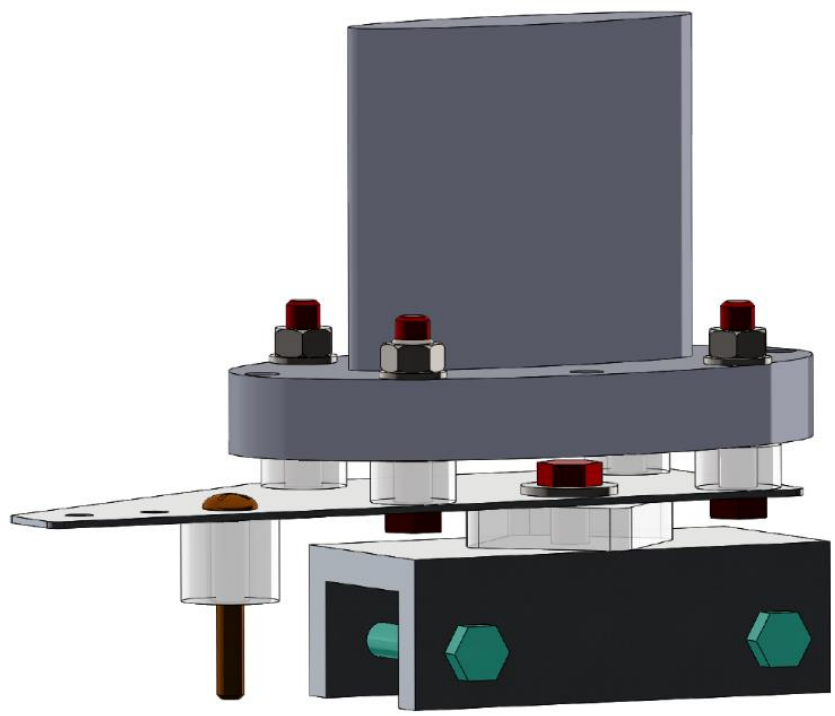

Figure 18. Mounting assembly overview with linear drive mounting plate shown (top).

A $1.6 \mathrm{~mm}$ thick sheet metal adapter plate was designed to act as an interface between the test model and the linear drive mounting plate. Four M8 bolts attached the adapter plate to the linear drive mounting plate. Four 3D printed cylindrical T-Glase spacers were used to provide clearance for the main M8 pivot bolt and the pivot washer. This pivot point was slightly offset in the lateral direction in an effort to center the model in the wind tunnel and to ensure that the center of gravity of the model was directly below the linear drive mounting plate, thereby limiting undesirable rotational forces on the model. This offset mounting also served to keep the feathers as far from the tunnel wall as possible at higher angles of attack. Two of the four mounting nuts were M8 nyloninsert lock nuts, whereas the remaining two were standard M8 nuts. Each nut rested on a washer to prevent damage to the linear drive mounting plate. 
Mounted to the main pivot bolt was a machined pivot block, which was held in place by a M8 nylon-insert lock nut tightened against a washer. The pivot block and mounting plate were separated by a square 3D printed $\mathrm{T}$-Glase spacer designed to minimize any lateral play in the mounting assembly and provide clearance for the four mounting bolts during pivot block rotation. A pinned connection between the pivot block and test model utilizing two 5/16-24 bolts and standard nuts was chosen to allow easy assembly and disassembly, as well as to limit any sharp edges or unnecessary loads on the 3D printed parts. Aside from the four M8 mounting bolts, which were part of an existing hardware kit, all bolts were fully threaded. This was considered acceptable as a cost reduction measure due to the low expected loads.

\subsubsection{Angle of Attack Control}

A pattern of three holes at the forward end of the mounting plate were used to control the model's angle of attack. A single 1/4-28 pan head machine screw was used as a pin to align the mounting plate hole with its respective hole in the model to select $0^{\circ}, 5^{\circ}$ or $10^{\circ}$ angle of attack. During testing, the machine screw was held in place and faired over using aluminum tape. A 3D printed T-Glase spacer was also used at this location to help align the model and prevent rocking during testing. Figure 19 presents a top-down view of the model at each angle of attack. 

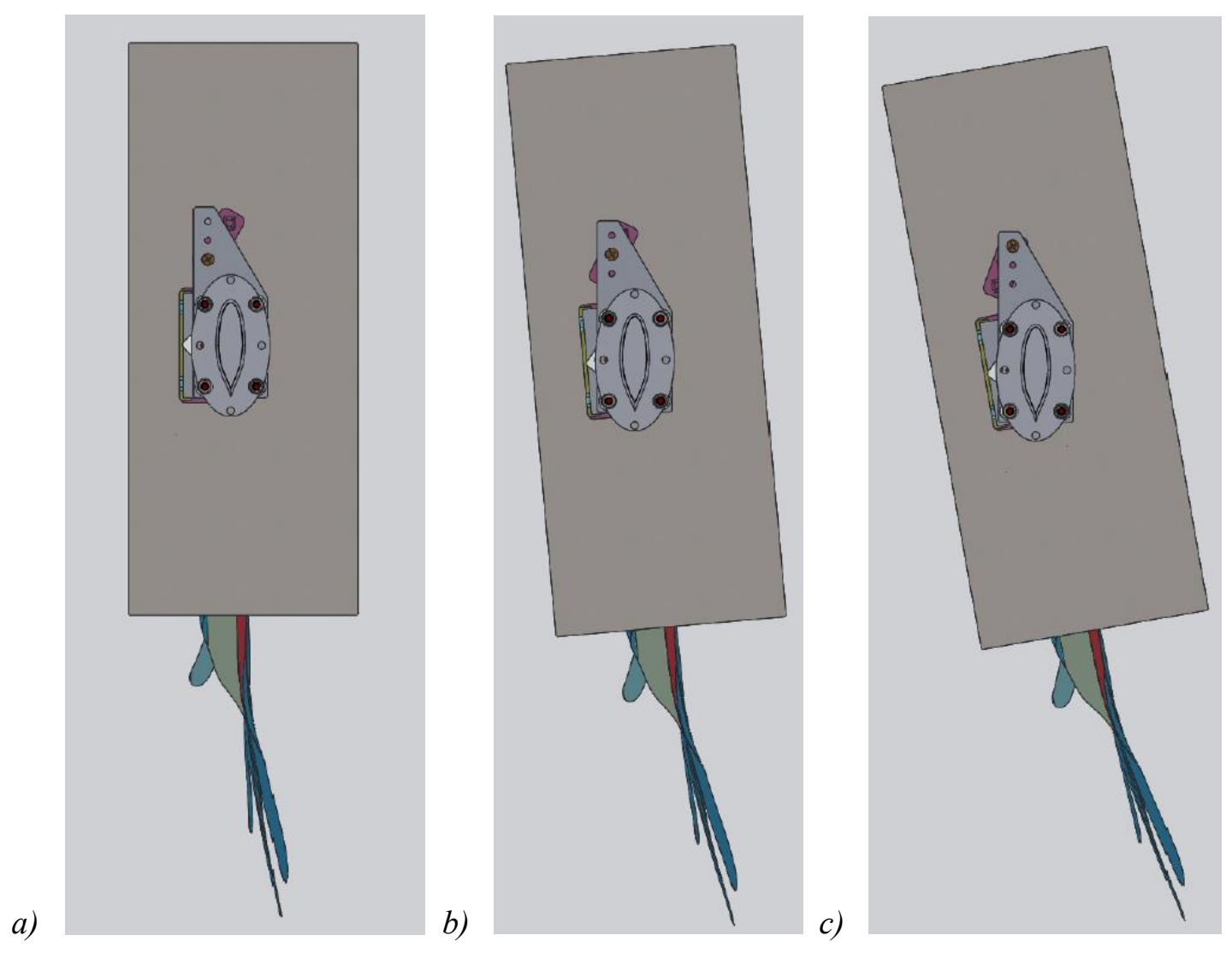

Figure 19. Overview of the final model at a) $0^{\circ}$, b) $5^{\circ}$ and c) $10^{\circ}$ angle of attack. Air flow is from top to bottom. 


\section{AERODYNAMIC TEST MODEL CREATION}

\subsection{D Printing Overview}

3D printing (also sometimes referred to as additive manufacturing) has existed in some capacity for the last thirty years, though consumer-level 3D printers have only recently become available at an affordable price. "Additive manufacturing" acts as an umbrella term for several various technologies such as stereolithography, selective laser sintering (SLS) and fused deposition modeling (FDM), among others. The majority of consumer-level 3D printers employ some variation of the FDM process, where a spool of plastic filament is gradually unwound, heated by the print head and extruded in successive layers to form the final three-dimensional component.

Early conceptual primary feather prints were carried out using both an Ultimaker $2+$ and a LulzBot TAZ 5 3D printer. However, various mechanical difficulties and poor print quality drove the decision to print the final production components using a LulzBot TAZ 6 3D printer. The TAZ 6 is a commercially available 3D printer designed and assembled by Aleph Objects, Inc. with a usable printing volume of $0.28 \mathrm{~m} \times 0.28 \mathrm{~m} \times 0.25 \mathrm{~m}$. Printing is accomplished using a moveable print bed, which translates forward and back, as well as a mobile print head, which moves left and right as well as vertically. The TAZ 6 is able to print using a number of industry standard plastics and manufactures components using a FDM process. Because of the finite size of the print head $(0.4 \mathrm{~mm})$ and the fact that printing occurs in successive layers, there is an inherent printing "resolution" for any component created using this process. Depending on the exact geometry, this resolution can be clearly noticeable on curved surfaces and results in some degree of surface roughness. Issues related to the surface roughness of printed components and surface finishing operations will be revisited in Section 6.5.

The TAZ 6 runs on a variant of the common G-code numerical control programming language. After importing a STL model of the part to be printed and choosing the appropriate settings, the necessary G-code instructions (often referred to as the "toolpath") are generated by 
specialized software. The G-code can then be uploaded to the printer either via USB or by using a Secure Digital (SD) data storage card.

\subsection{Materials}

Early conceptual test prints were carried out using PLA (polylactic acid) on an Ultimaker $2+$. Due to its low cost and reasonable print characteristics, PLA is one of the most common thermoplastics currently in use for commercial-grade 3D printing. Unlike many 3D printing plastics, which are petroleum-based, PLA is composed of organic material harvested from plant matter. However, printed PLA can exhibit brittleness, making it unsuitable for tall and thin components. Severe printing issues created by a combination of low-grade PLA and technical issues with the Ultimaker $2+$ printer eventually spurred additional research into alternative plastics and printers.

After switching to the LulzBot TAZ 5 printer, additional conceptual prints were generated using a high-grade ABS (acrylonitrile butadiene styrene) plastic manufactured by IC3D. While one of the most widely used plastics in 3D printing, ABS is extremely susceptible to splitting and warping when used to print larger parts. Temperature gradients created within the model during the printing process can cause non-uniform contraction or expansion of the material, which may cause print layers to pull apart from one another or cause the entire print to detatch from the print bed, severely impacting the structural integrity of the finished component. This can be addressed to some degree by printing inside of an enclosure, which keeps the ambient temperature both higher and more consistent. While preliminary test components were printed in a heated room without an enclosure, subsequent test prints were carried out using an enclosure constructed out of sheets of Coroplast, a corrugated plastic.

On top of the technical challenges involved in obtaining high quality prints using ABS, additional concerns remained regarding the dimensional accuracy of the final parts due to the 
likelihood of warping and splitting. These issues prompted additional research into high performance plastics. After an exhaustive comparison process, the decision was made to switch to T-Glase, a PET (polyethylene terephthalate) polymer manufactured by Taulman3D. In comparison to ABS, T-Glase exhibits virtually no warping or splitting when printed with the appropriate settings, even when printing without an enclosure. While the shrinkage factor post-print is low, TGlase has nonetheless been known to crack and damage print beds when allowed to cool after the printing process due to its extremely high strength. This is also due in part to Taulman3D's suggestion to use a standard glue stick to coat the print bed in a thin layer of PVA (polyvinyl acetate), which dramatically enhances the ability of the component to adhere to the print bed. To combat these issues, the final G-code was manually edited to command the print bed to cool to $40^{\circ} \mathrm{C}$ rather than ambient temperature to limit the contraction of printed components before removal from the print bed. After chipping one or more corners free of the bed using a small chisel, high strength dental floss was then wedged underneath the exposed corners and used to quickly pry the remainder of the model from the print bed. In extreme cases, the print bed was briefly heated to 85 ${ }^{\circ} \mathrm{C}$ to soften the layer of PVA, allowing more efficient use of the dental floss removal method. 

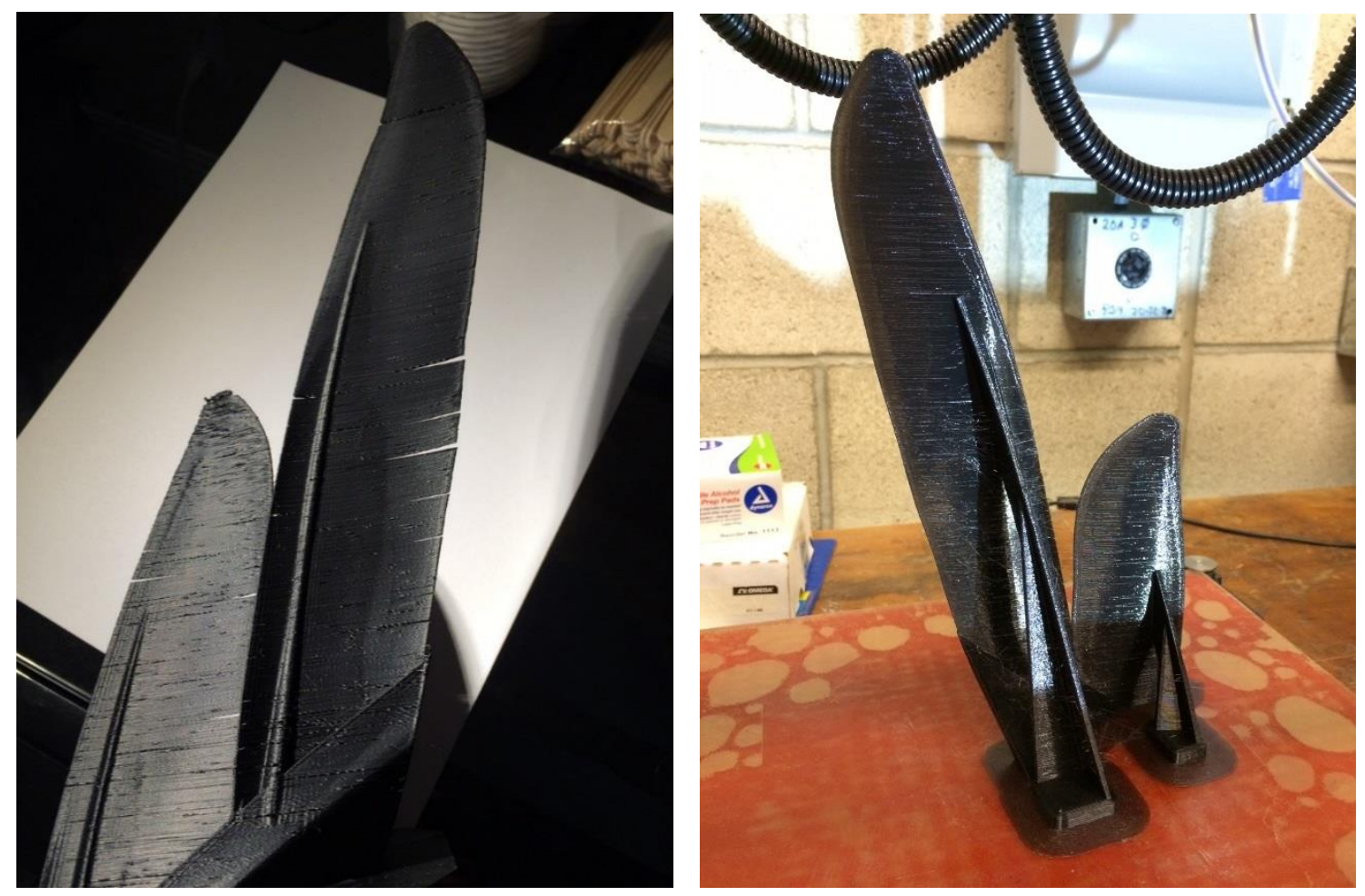

Figure 20. A comparison between identical parts printed in ABS (left) and T-Glase (right).

Warping and splitting between print layers is clearly visible in the ABS print.

\subsection{Printing Software and Operation}

Toolpaths for early conceptual prints using the Ultimaker 2+ and LulzBot TAZ 5 were created using Cura, a free open-source 3D CAD to toolpath converter (commonly called a "slicer") currently maintained by Ultimaker. Cura allows the user to set up prints, specify various printing options and export the resulting G-Code instructions defining the operation of the printer. However, the toolpaths for the final 3D printed parts were created using Simplify3D. While conceptually similar to Cura, Simplify3D offers a significantly greater number of options to control the creation and quality of a print. While an exhaustive list of every print setting used will not be discussed here, a review of some of the key settings leading to the high-quality prints used for the final test model is presented below. Because of the structural challenges faced by the parts including feathers, 
many settings often differed between these parts and those of the main wing body. Both settings will be discussed when applicable.

Temperatures: Both the temperature of the extruder nozzle and the print bed are of prime importance when using a FDM printer. Excessively high temperatures can cause plastic to ooze from the extruder nozzle or cause poor adhesion to the print bed. Alternatively, low temperatures will prevent the plastic from adhering to previous layers or the print bed, producing a component with poor structural integrity and surface finish. After several test prints, an extruder nozzle temperature of $225{ }^{\circ} \mathrm{C}$ and a print bed temperature of $70{ }^{\circ} \mathrm{C}$ were found to provide the most consistent quality when using T-Glase. As previously discussed, the final G-Code was manually modified to command the printer plate to cool to $40{ }^{\circ} \mathrm{C}$ instead of ambient to prevent cracking or chipping of the build plate post-print.

Print Speed: Print speed refers to the maximum lateral movement of the extruder nozzle during the printing process. A maximum print speed of $25 \mathrm{~mm} / \mathrm{s}$ was used when printing with T-Glase. While this is relatively slow when compared to most commercially available 3D printing plastics, it is in the realm of speeds suggested by Taulman3D when printing with T-Glase. Testing at higher speeds often resulted in degraded structural integrity and surface finish.

Retraction: Retraction occurs when a printer retracts filament instead of extruding. This draws the heated filament back into the nozzle by a specified amount to prevent oozing or stringing of the heated filament during horizontal nozzle movements, which can interfere with the operation of the printer or result in a poor surface finish. When using a retraction of $10 \mathrm{~mm}$ with T-Glase, oozing and stringing was nearly eliminated. An option in Simplify3D to only allow retraction when crossing between profiles of the component was also enabled. This forces the printer to extrude 
continuously without retraction over each closed profile on a given layer, reducing the number of retraction movements and hence starts/stops of the extruder.

Brim: Brims are often used to increase the adhesion between a printed component and a print bed and are typically one extrusion layer tall. A brim may extend for many layers laterally around the model depending on the needs of the particular part. While brims are generally used to help anchor a component to the print bed, they can also be used to "prime" the extrusion process when printed from the outer profile inwards, ensuring continuous extrusion by the time printing of the actual component begins. For components with a relatively small base, 10 brim layers were used. Components with larger base areas required 15 brim layers to maintain adhesion.

Starting Location: The starting location, or where along the profile the printer begins printing a new layer, can either be randomized or set to a specific location. In general, the starting location was placed at a convenient corner of each component to ensure smooth and consistent extrusion along the entirety of the profile. Any irregularities related to over- or under-extrusion at the start point were removed post-print using sandpaper. For components with feathers, the starting location of each profile was chosen to be the leading edge to prevent printing directly over the large overhang present at the trailing edge.

Infill: While it is possible to create solid plastic components using 3D printing processes, this is generally inefficient from a cost, time and material usage standpoint and often results in noticeably warped components due to strong temperature gradients within the component. Many toolpath generators offer options to create simplified structural infill patterns to reduce print time and cost. Simplify3D includes multiple infill options, such as rectilinear, grid, triangular, wiggle, fast honeycomb and full honeycomb. 
Each infill type has various advantages and disadvantages. A 35\% triangular infill was used for all components containing feathers. This was to ensure sufficient structural rigidity of the part while minimizing the print time and material usage. Components of the main wing body were printed using a $15 \%$ full honeycomb infill, which offers the best strength vs. material used ratio among the available options. The only available infill stronger than full honeycomb is the triangular infill, though this is slightly less efficient in terms of print time and material usage. However, while strength was certainly a concern, it was determined that print time and material usage were higher priority for the majority of print jobs due to the relatively low loads expected to be placed on the model.

Perimeter Layers: The number of perimeter layers controls how thick the outer wall of the printed part will be before infill is applied. For components including feathers, this was set to 5 layers. This resulted in solid feathers with no infill, though infill was still applied to the thicker base of each feather component. The remaining components were printed with 3 perimeter layers as this offered an ideal compromise between strength, material usage, print time and overall wall thickness.

In addition to the software settings discussed above, it was also discovered that the tension in the printer idler assembly played a large role in print quality. Many 3D printers utilizing a FDM process, such as the TAZ 6, draw plastic filament into the extruder nozzle using an extruder filament drive gear. A variable tension idler pulley forces the filament against the drive gear to maintain positive pressure and ensure that the drive gear is able to consistently grip the filament. An overly tight idler assembly can crush the plastic filament between the idler pulley and the drive gear, making extrusion inconsistent when retraction operations are used. This issue occurred during early testing with the LulzBot TAZ 6 and resulted in inconsistent extrusion and unusable printed parts with poor surface finish quality. 


\subsection{Support Structures}

For components requiring extra support during the $3 \mathrm{D}$ printing process, either due to overhangs or poor structural rigidity (e.g. feather components), a series of support structures was designed. Each structure featured a $1 \mathrm{~cm}$ tall square base with rounded corners, which provided a solid foundation for the structure and ensured proper adhesion to the print bed. Above the foundation, truss-like structures supported the component as necessary. These were tapered to provide sufficient support while minimizing print time and material usage. Figure 21 provides an overview of the support structures designed for the feather components.
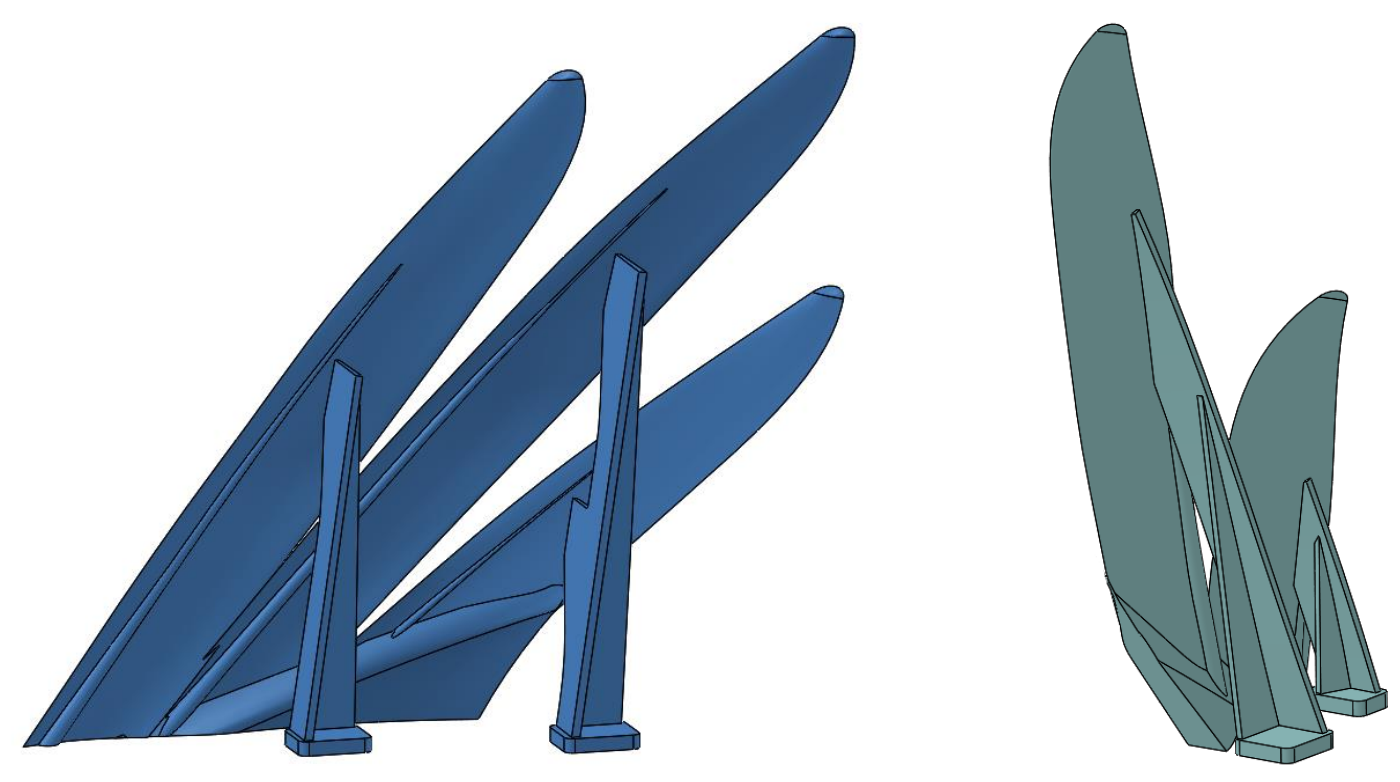

Figure 21. Detail views of the support structures designed for each feather component.

\subsection{Surface Finishing}

Commercially available $3 \mathrm{D}$ printers can typically produce a consistent and reasonably high quality surface finish on printed components depending on print settings and material selection. As a consequence of the way FDM printers operate, curved surfaces are often slightly stepped due to the finite thickness of the filament used to create each layer of the component. Because of this, 
additional refinement was required to improve the surface finish of the final test model such that it was suitable for aerodynamic testing.

Before addressing the surface finish of each component, any support structures required for $3 \mathrm{D}$ printing were first removed using a rotary tool. A cutting wheel was used to remove the support structures, leaving approximately $3 \mathrm{~mm}$ of material left. A sanding wheel and $3 \mathrm{M} 150$ grit high performance sandpaper were then used to remove the remaining material. Additional sanding was carried out to ensure that bonding interfaces on plastic parts were flat and provided a consistent bonding surface, as well as to remove any surface defects or protrusions from the remaining surfaces. A final sanding pass using 3M 400 grit sandpaper was carried out to smooth out any ridges left by the 150 grit sandpaper. Sandpaper was typically soaked in water before sanding in an effort to prevent plastic particulates from becoming airborne and to carry particulates away from the sanding area.

After the surface finish of each 3D printed component was improved as much as possible using sandpaper, steel dowel interface pins were inserted into the respective mounting holes and the components bonded together. It should be noted that standard adhesives such as two-part epoxies and hot melt adhesives are generally ineffective at bonding polyethylene plastics due to the plastic's low surface energy. This can cause the adhesive to bead up on the bonding surface instead of wetting the surface as intended, resulting in poor integrity of the resulting bond. Recent research has yielded several advanced adhesives designed for use with low surface energy plastics. ${ }^{27}$ One such consumer-grade product is the two-part Loctite Plastics Bonding System (PBS), which uses a heptane primer pen in conjunction with cyanoacrylate adhesive to create a mechanically durable bond. All bonding carried out during the assembly process, including plastic-to-plastic and plasticto-metal bonds, was performed using Loctite's PBS.

After the adhesive had fully cured, any remaining gaps in the model were filled by hand with Apoxie Sculpt, a self-hardening two-part synthetic modeling clay produced by Aves Studio. 
In addition to its ability to fill gaps and create a durable bond over a wide variety of surfaces and materials, Apoxie Sculpt can be sanded and painted after curing. After a 24-hour cure time and additional sanding to smooth the Apoxie Sculpt, a coat of XTC-3D was applied to all exterior surfaces using a foam brush with a chiseled edge. XTC-3D, a Smooth-On product, is a specially formulated two-part epoxy that provides a durable, smooth, sandable and paintable finish for 3D printed parts. Only a thin layer of XTC-3D was applied, just enough to fill any remaining striations or steps left by the $3 \mathrm{D}$ printing process. While extra coating would likely have increased the durability of printed parts, an effort was made to keep the outer dimensions of all models as similar to the intended dimensions as possible. After a four hour cure time, any non-uniform areas in the coating were sanded using a combination of 150 grit and 400 grit sandpaper until all faces of the model were smooth to the touch.

Finally, the assembled model was sprayed with a coat of Krylon black chalkboard paint. After sanding the initial layer of paint using 400 grit sandpaper, a final coat was applied, which provided a smooth matte black surface finish. Similar to the coat of XTC-3D, only very thin layers of paint were applied to avoid excessive deviation from the original part dimensions. Matte chalkboard paint was chosen to prevent scattering of ambient light during operation of the wind tunnel laboratory's particle image velocimetry (PIV) system. While ultimately not used for this project, future projects involving this model will likely make use of the PIV system. No XTC-3D or spray paint was applied to the interface surfaces between parts so as not to alter the part dimensions any more than necessary and to allow proper bonding of the parts.

\subsection{Machined Components}

While the majority of components used in the final test assembly were $3 \mathrm{D}$ printed, an additional three components were also machined out of aluminum 6061-T6 alloy. These include 
the main pivot block, the mounting plate and the splitter plate. Engineering drawings of each component may be found in Appendix B.

While the pivot block was machined from bar stock on a Haas TM-1 toolroom mill, the remaining sheet metal components were cut and drilled by hand using 1:1 scale flat patterns provided in the engineering drawings. $1.6 \mathrm{~mm}$ thick sheet metal was used for both the mounting plate and splitter plate, which provided sufficient strength and rigidity while minimizing weight, cost and the projected area exposed to the flow. Each component was spray painted with Krylon black chalkboard paint to mimic the finish of the wing body assembly. Aside from the dimensions defining hole patterns and their respective hole diameters, machining tolerances were kept relatively loose to simplify the part creation process and reduce overall fabrication time.

To prepare the splitter plate for bonding to the wing body, \#3 coarse grade steel wool was used to remove any paint from the bond area and to prepare the aluminum surface for bonding. After cleaning the bond area with isopropyl alcohol and allowing to air dry, the splitter plate was bonded to the wing body using the Loctite PBS (with additional cyanoacrylate to ensure full coverage) and allowed to cure. Any gaps between the wing body and the splitter plate were faired over using black electrical tape.

\subsection{Final Assembly}

Figures 22 and 23 present an overview of the final assembly mounted in the low speed wind tunnel. Assembly drawings detailing the main assembly and each of the three subassemblies can be found in Appendix B. A complete Bill of Materials is included in each assembly drawing for reference. 


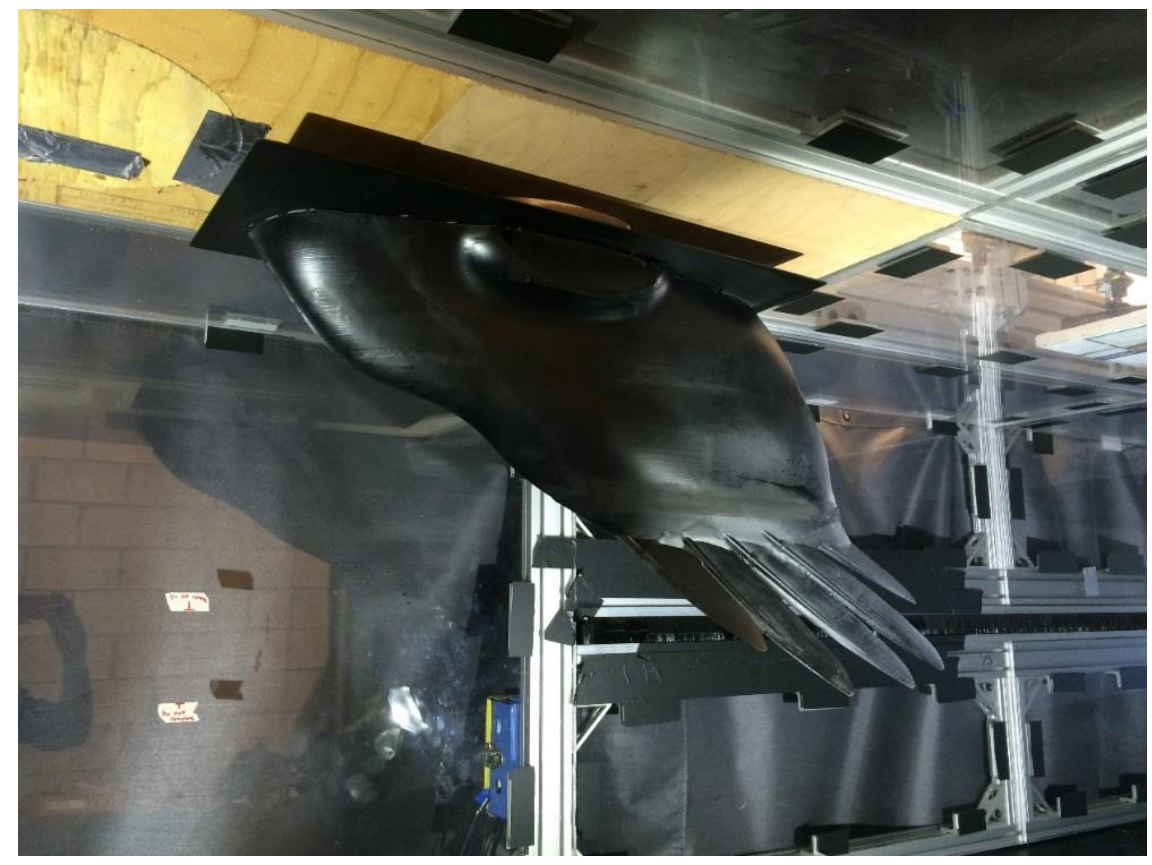

Figure 22. Final aerodynamic test model mounted in the Cal Poly low speed wind tunnel. Side view.

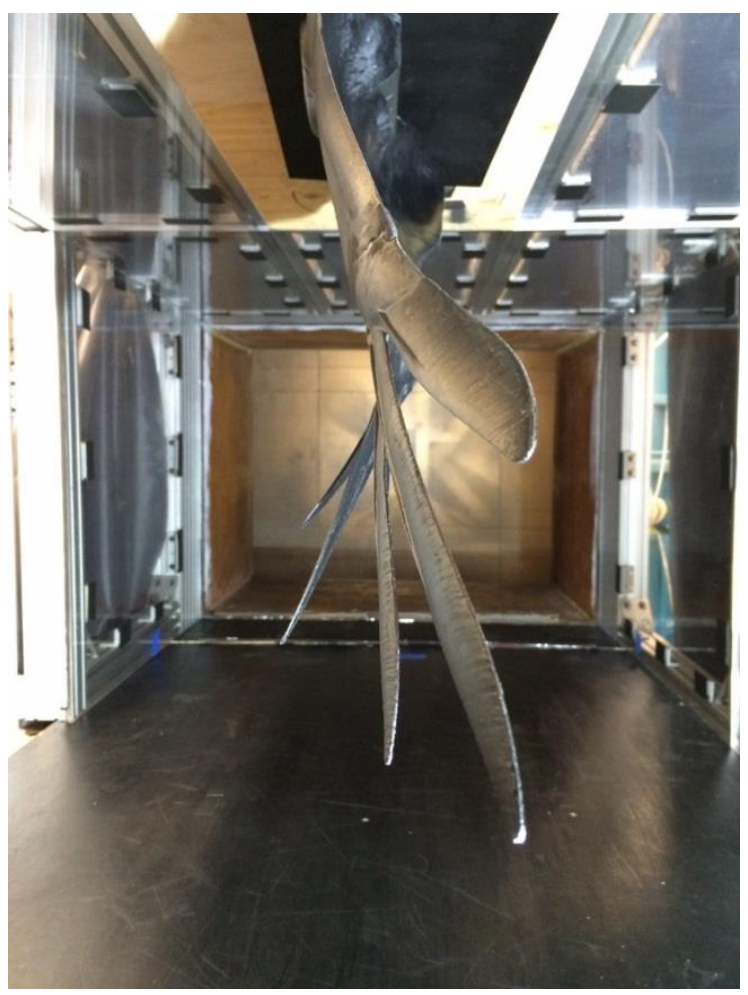

Figure 23. Final aerodynamic test model mounted in the Cal Poly low speed wind tunnel. Rear view. 


\subsection{Wind Tunnel Overview}

Aerodynamic testing was conducted at the Cal Poly aerospace department low speed wind tunnel research laboratory on the Cal Poly campus.

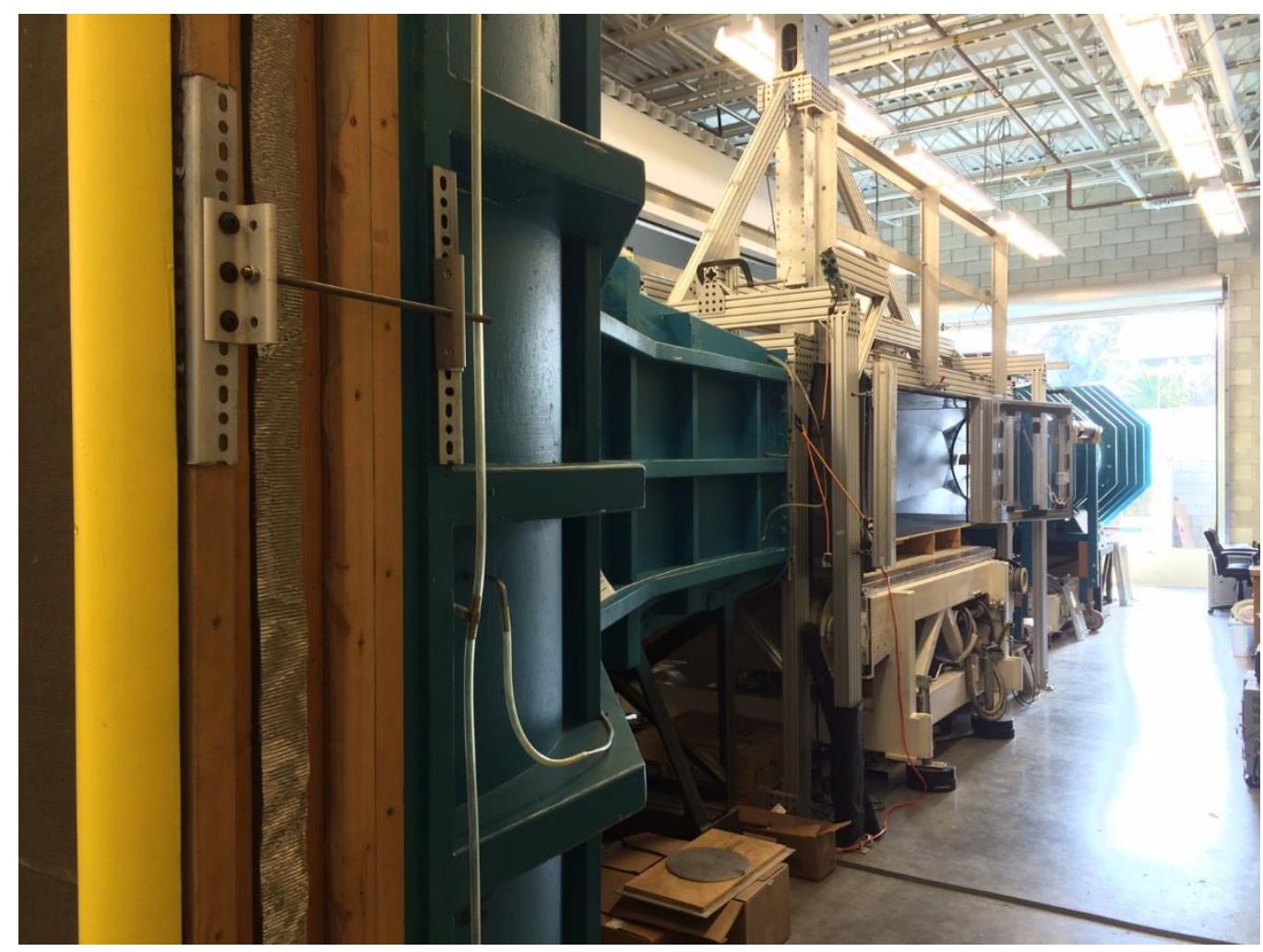

Figure 24. The Cal Poly low speed wind tunnel with the test section doors open. Air flow is from left to right.

The rectangular test section of the low speed wind tunnel is $1.15 \mathrm{~m}$ wide by $0.88 \mathrm{~m}$ tall and spans a total length of $4.27 \mathrm{~m}$. The test section walls are composed of a T-slot aluminum extrusion skeleton with acrylic walls to enable the inspection of the test section during model setup and test operations. The tunnel is an open circuit design with rectangular inlet dimensions $2.75 \mathrm{~m}$ by 3.65 $\mathrm{m}$, resulting in a contraction ratio of approximately 10 . Flow straightening is accomplished using a 
series of wire mesh screens sandwiching a $0.05 \mathrm{~m}$ thick honeycomb flow straightener mounted at the inlet, with one screen immediately upstream of the flow straightener and two immediately downstream. A pressure ring system at the test section entrance provides instantaneous static pressure readings whereas a total pressure probe immediately downstream of the inlet provides total pressure readings. These readings are utilized by a custom LabVIEW VI along with current temperature, pressure and ambient air density readings to continuously calculate the dynamic pressure and flow velocity in the test section during test operations.

The fan is driven by a $125 \mathrm{HP}$ Allen-Bradley inverter duty electric motor (model number ENP44G0365N-MG) via a belt drive system. Fan speed is controlled by specifying the desired revolutions per minute (RPM) of the motor on an Allen Bradley PowerFlex Variable Frequency Drive. The fan utilizes 9 blades and is mounted downstream of the test section to prevent the fan wake from interfering with test measurements. The maximum speed of the tunnel is approximately $45 \mathrm{~m} / \mathrm{s}$.

\subsection{Linear Drive}

The final test model was secured to the mounting plate of the wind tunnel's linear drive system during testing. The linear drive system is mounted above the wind tunnel and allows for vertical translation of models within the test section. While the final model was not translated during testing, the linear drive was lowered during assembly and disassembly for tool clearance purposes and ease of access to the mounting plate. Actuation of the linear drive was accomplished using an existing custom LabVIEW VI. The front edge of the mounting plate is approximately 1.25 $\mathrm{m}$ from the front of the test section, placing the linear drive slightly forward of the test section center. 


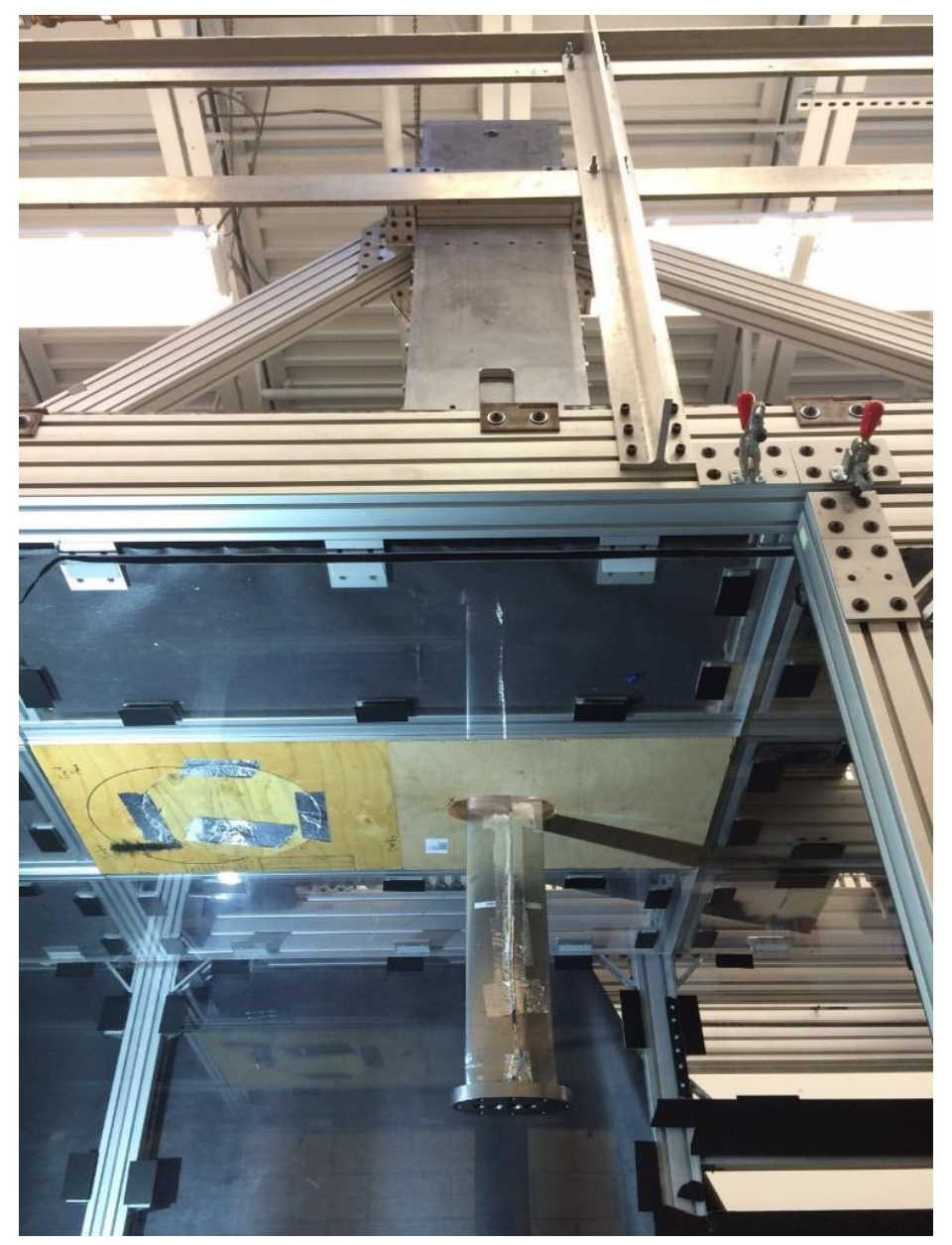

Figure 25. Overview of the linear drive system with mounting plate extended.

\subsection{Line Laser}

A Laser Modules M-18B532-X-GL line laser was used during visualization experiments. The $5 \mathrm{~V}$ line laser generated a green laser light at a wavelength of $532 \mathrm{~nm}$ and was held in place by a standard clamp stand. The line laser setup was placed on an acrylic viewport above the wind tunnel test section, allowing the line laser to fire directly down into the test section. The twodimensional laser sheet was oriented normal to the wind tunnel air flow and placed at either $0.1 \mathrm{~m}$ or $0.2 \mathrm{~m}$ downstream of the tip of the fourth feather based on testing requirements. While several observation locations were investigated during preliminary visualization experiments, observation at $0.1 \mathrm{~m}$ and $0.2 \mathrm{~m}$ downstream led to the most consistent and well-formed vortex structures. 


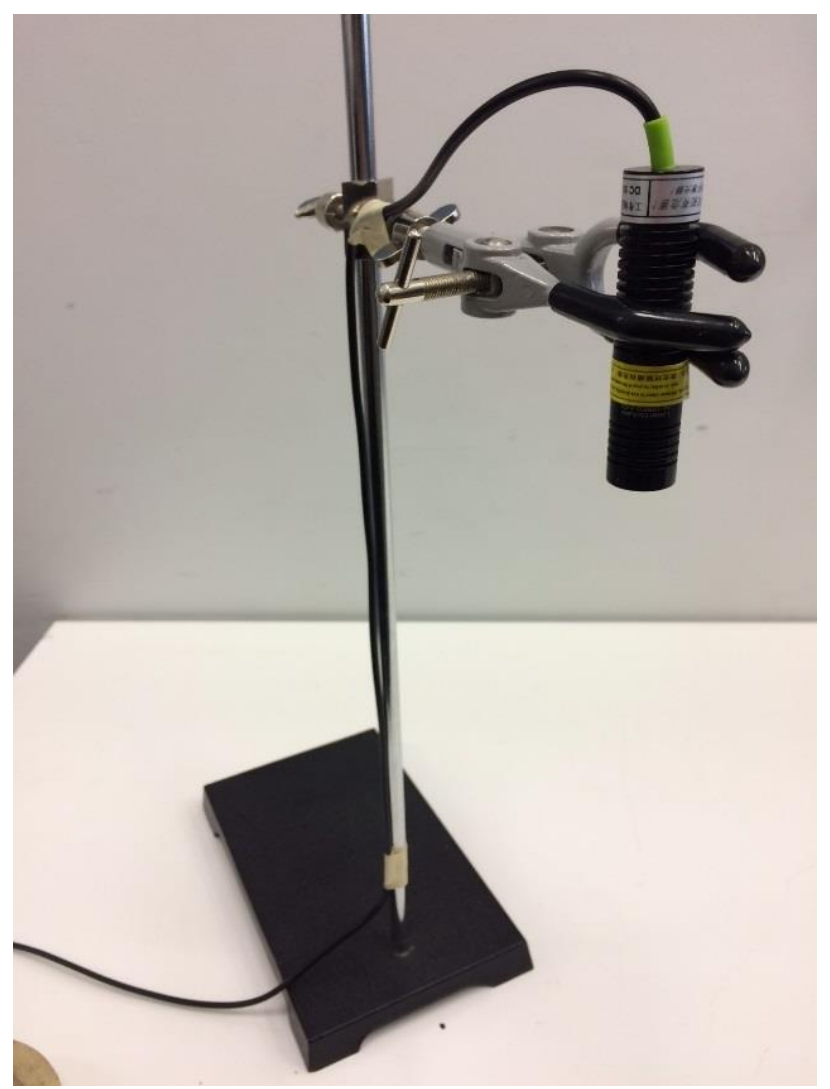

Figure 26. Line laser setup.

\subsection{Fog Machine}

Fog used for flow visualization purposes was generated by a Rosco Alpha 900 heated fog machine using Froggy's High Density Bog Fog clear fog fluid, which is composed of a mixture of low molecular weight glycols and deionized water. Once pre-heated, the fog machine is controlled by a simple rocker switch at the end of a $9 \mathrm{~m}$ cord. The fog machine vaporizes the fluid by using an electric pump to move the fluid across an internal $1000 \mathrm{~W}$ heat exchanger system, resulting in a continuous stream of high density fog.

Initial testing revealed that the electric pump introduced significant vorticity to the resulting fog plume. A nozzle was fashioned from an 8 gallon waste bin and two layers of circular flow straightening mesh in an effort to both condition the flow and expand the overall fog plume. 
The machine was also cleaned before operation using Chauvet fog machine cleaner, a vinegarbased cleaning solution designed to remove deposits from the heater core.

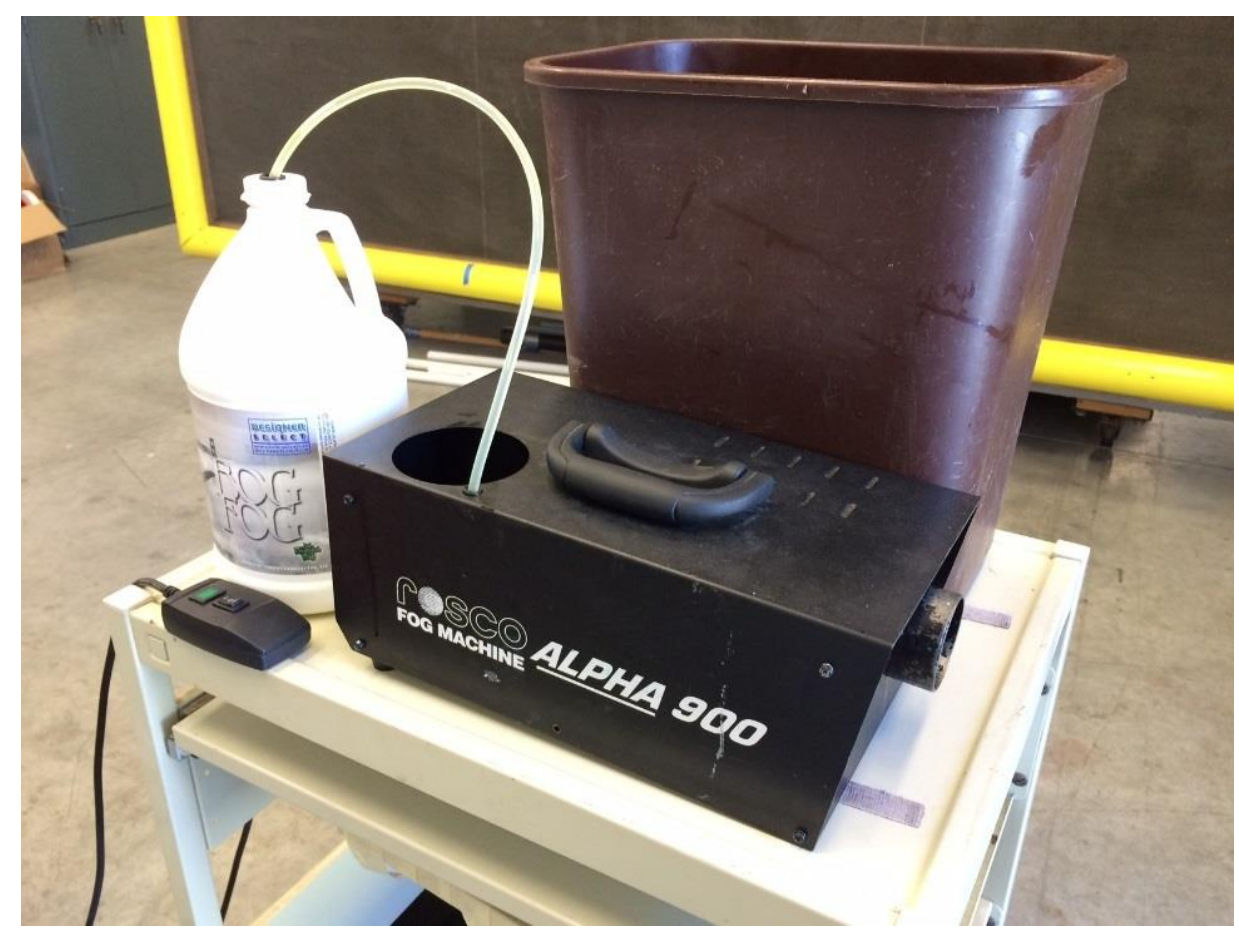

Figure 27. Fog machine setup.

\subsection{Traverse System}

The wind tunnel's traverse system is rigidly mounted to the test section of the wind tunnel and allows for translation of measurement devices or models during test operations. The system provides linear movement along all three axes using a series of stepper motors and is controlled via a custom LabVIEW VI. The traverse was used to sweep a total pressure rake laterally and vertically in the wake of the test model to obtain two-dimensional maps of the total pressure. Later testing utilized the traverse to sweep a total pressure probe through the wake of the model in a similar fashion.

A simple mount consisting of three 3D printed components was designed to secure the measurement devices to the traverse as no similar setup currently existed at the time of testing. The 
main structural component, or sting, was designed to extend forward from the traverse arm by 0.2 $\mathrm{m}$ to minimize potential flow interference created by the traverse arm. Due to the likelihood of the mount being utilized for future tests at higher speeds, the two main structural components were printed using high strength T-Glase. These were wedged together using a tapered interface and bonded together using Loctite's PBS. The final "cap" component was previously 3D printed using ABS and was repurposed from an existing assembly. The cap was secured in place using a series of four \#8-32 x 0.5 in machine screws and nylon insert nuts, while the entire assembly was mounted to the traverse arm using four $\# 8-32 \times 2.0$ in machine screws and nylon insert nuts. Figure 28 presents an overview of the assembly in Creo Parametric, while Figure 29 shows the assembly mounted to the traverse arm.

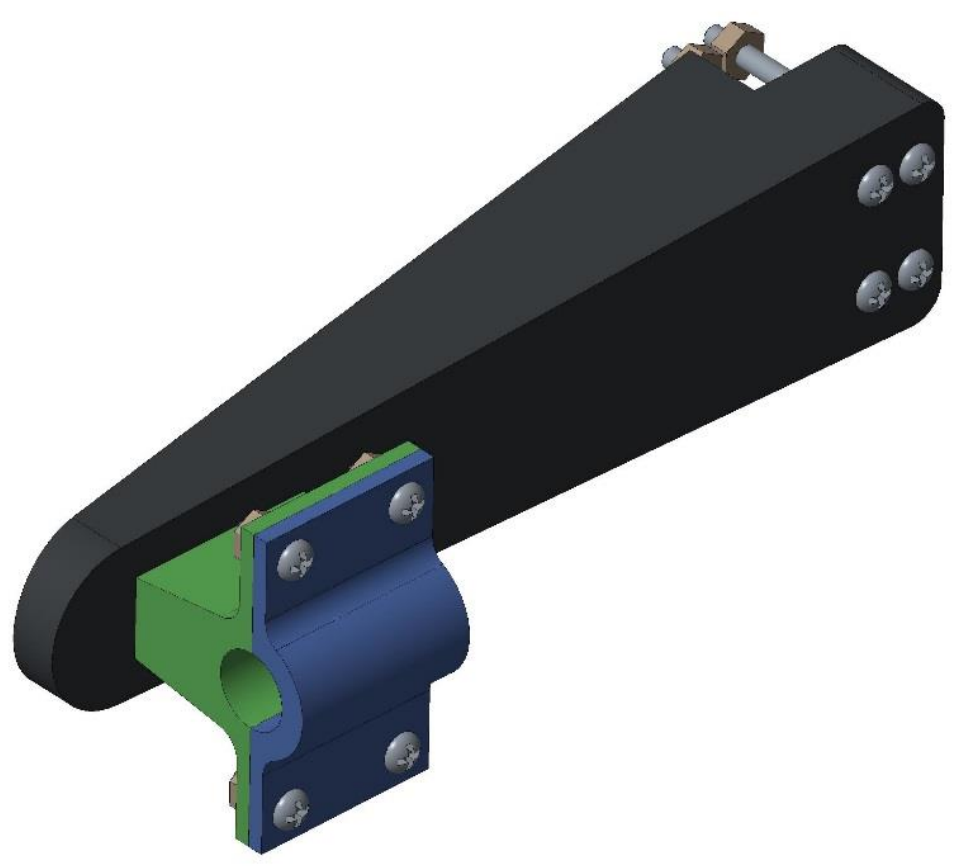

Figure 28. Measurement device mounting assembly. 


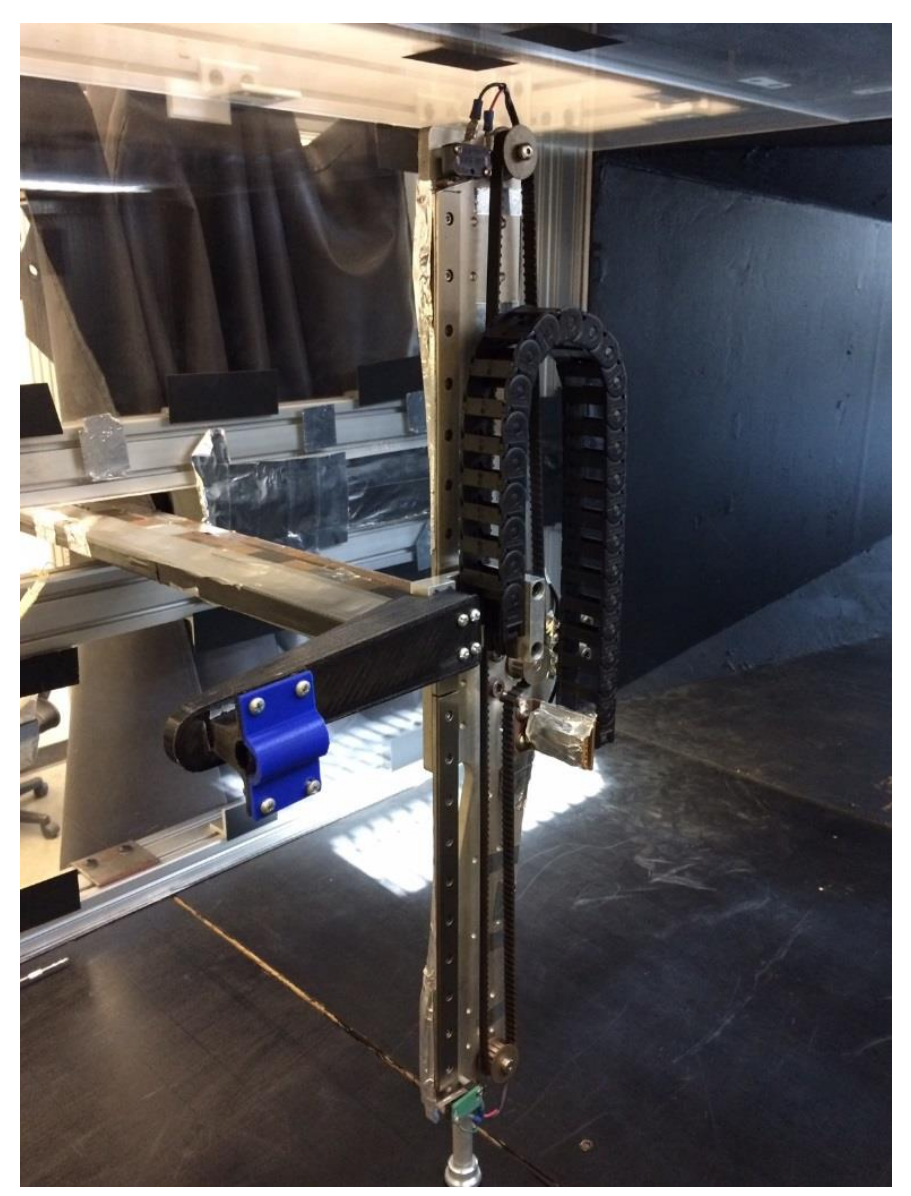

Figure 29. Overview of the traverse system with no measurement devices mounted.

\subsection{Scanivalve System}

Velocity measurement was accomplished using a Scanivalve ZOC33 pressure scanning system. Of the 64 available pressure ports, 32 have a range of 0.36 PSI ( \pm 0.15 FS accuracy), while the remaining 32 ports have a range of 1.0 PSI ( \pm 0.10 FS accuracy). Pressure values are computed by comparing measured pressure values to the pressure of a known source. In this case, the source is a direct connection to the wind tunnel laboratory's pressurized air line (60 psi). Voltage data generated by the pressure transducers is sent to a Scanivalve ERAD4000, which converts the pressure measurements into useful engineering data and forwards the data to a desktop computer via a wired Ethernet connection. The pressure measurement system is powered by a Scanivalve 
RPM1000 power supply. A custom LabVIEW VI records the sampled data and displays the dynamic pressure and tunnel flow velocity as calculated by the Scanivalve system in real time.

All pressure measurement tests in the wind tunnel were conducted using the same Scanivalve sampling configuration. 100 pressure samples were collected at each sampling location in the wind tunnel and were later averaged to create a single pressure measurement for each location. Each pressure sample was taken at a scanning period of $150 \mathrm{~ms}$ using 1 frame with 15 scans per frame, which resulted in each data point being an average of 15 individual scans. Using this sampling configuration, data from a single sampling location was acquired in approximately 33 seconds. Samples collected at each location were output as a MATLAB .m file for later postprocessing operations.

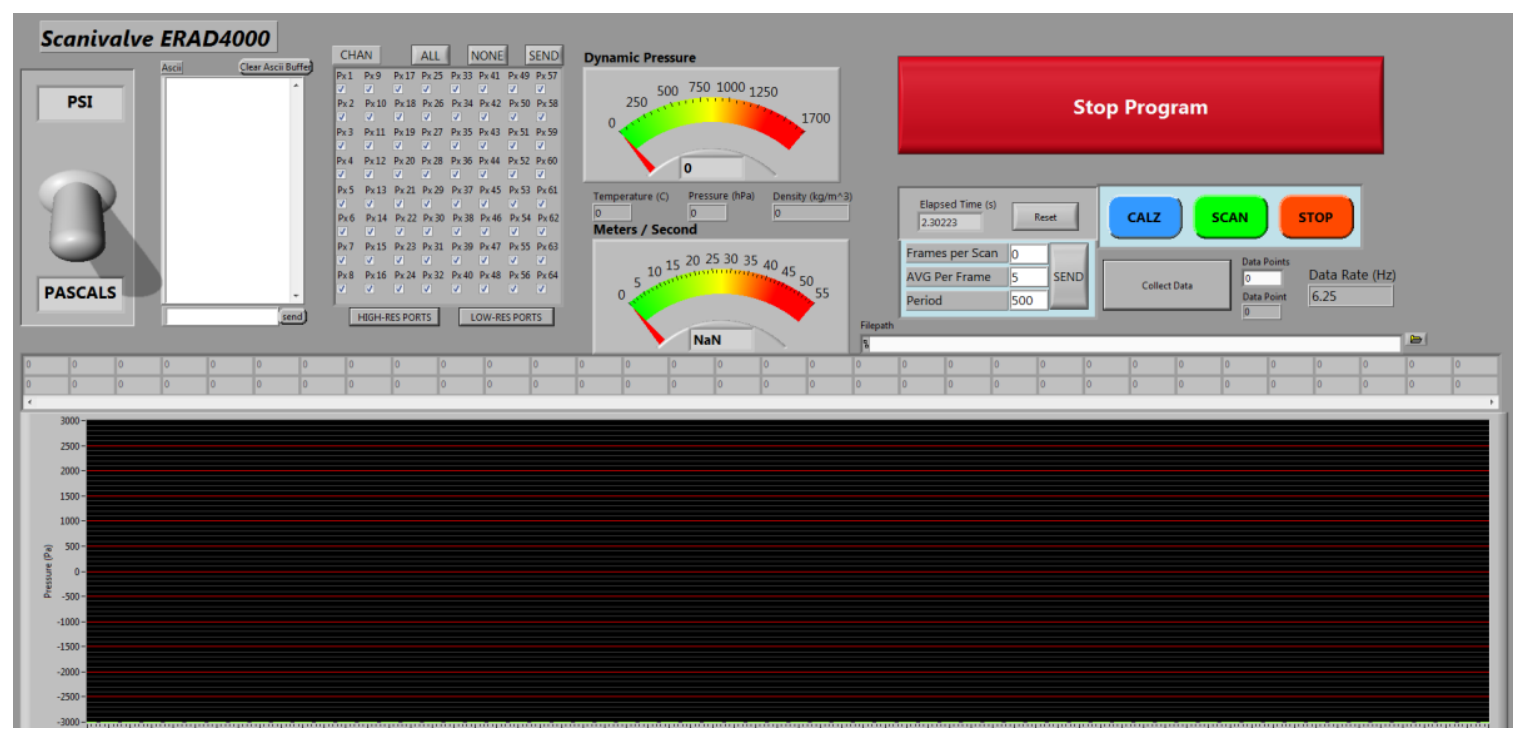

Figure 30. ERAD4000 LabVIEW interface.

\subsection{Pressure Rake}

The total pressure rake (occasionally referred to as a Pitot rake) used during testing is presented in Figure 31. The rake consists of a series of $201.6 \mathrm{~mm}$ diameter ports arranged in a 50 mm linear pattern. These metallic pressure ports converge to a circular pattern at the downstream 
end of the rake and are surrounded by a threaded enclosure. This threading allows for fine adjustment of the rake in the forward and aft directions when necessary, though this feature was not utilized during testing. Pressure tubing connected to the downstream end of each port carries the sampled air along the main traverse arm to the Scanivalve system mounted to the exterior of the test section.

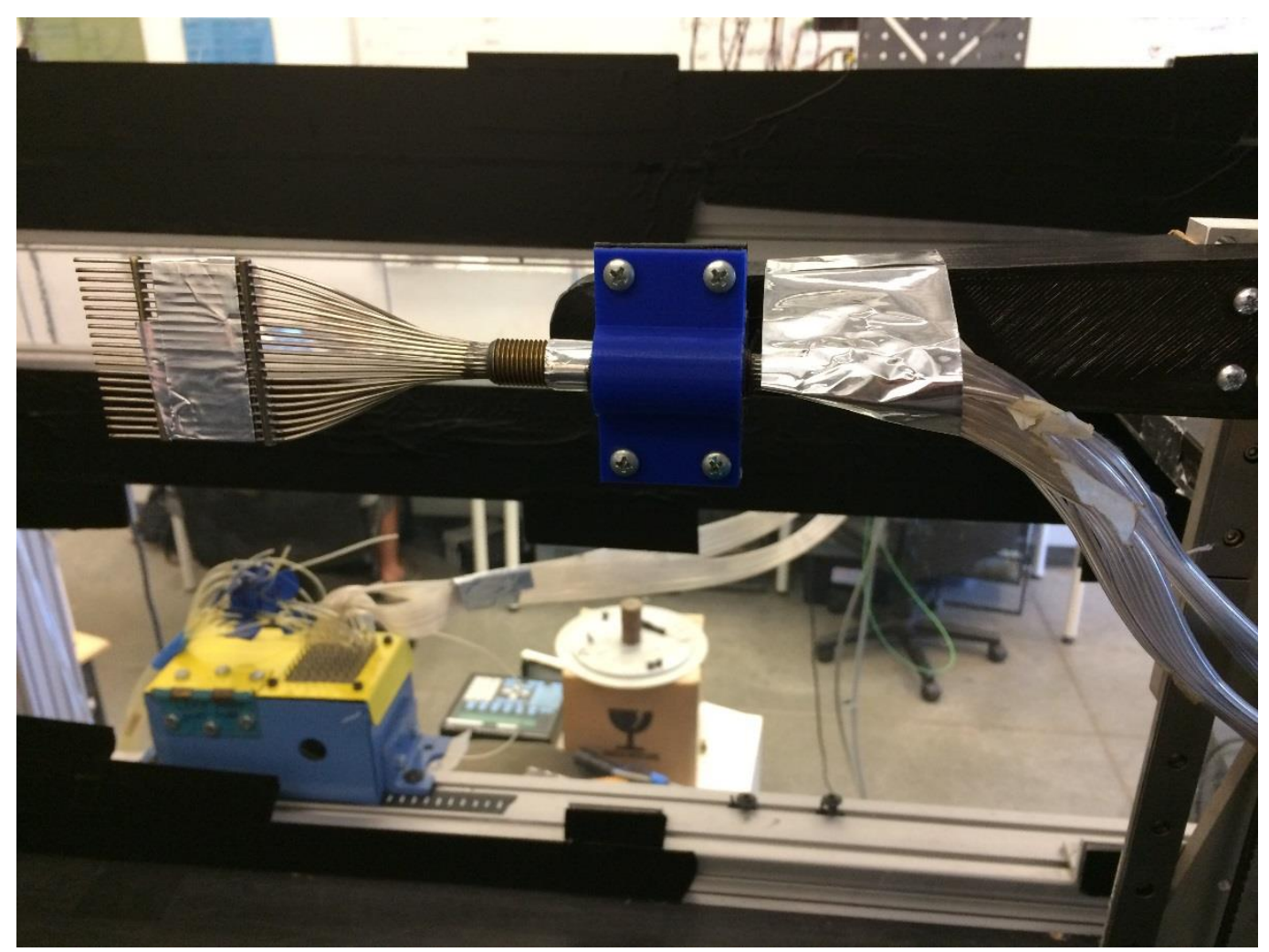

Figure 31. Pressure rake mounted to the traverse arm. Pressure tubing is routed across the arm of the traverse to the Scanivalve box (blue and yellow) shown in the background.

The pressure rake was swept through the wake the test model at a distance of $0.2 \mathrm{~m}$ downstream of the tip of the fourth feather to allow for correlation to earlier smoke visualization results. By nondimensionalizing using the chord of the scaled wing at the interface area between the wing body and primary feathers $(0.34 \mathrm{~m})$ for reference, a downstream distance of 0.59 chord 
lengths is obtained. Each lateral sweep was $240 \mathrm{~mm}$ in length and was split into 13 individual steps, resulting in a lateral step distance of $20 \mathrm{~mm}$. After moving the pressure rake upwards by $50 \mathrm{~mm}$, this lateral sweeping process was repeated. Data was collected at a total of 65 sampling locations. Figure 32 presents a grid outlining the pressure rake measurement area downstream of the model. The dimensions of $310 \mathrm{~mm}$ and $555 \mathrm{~mm}$ refer to the distance to the test section floor and wall, respectively. 

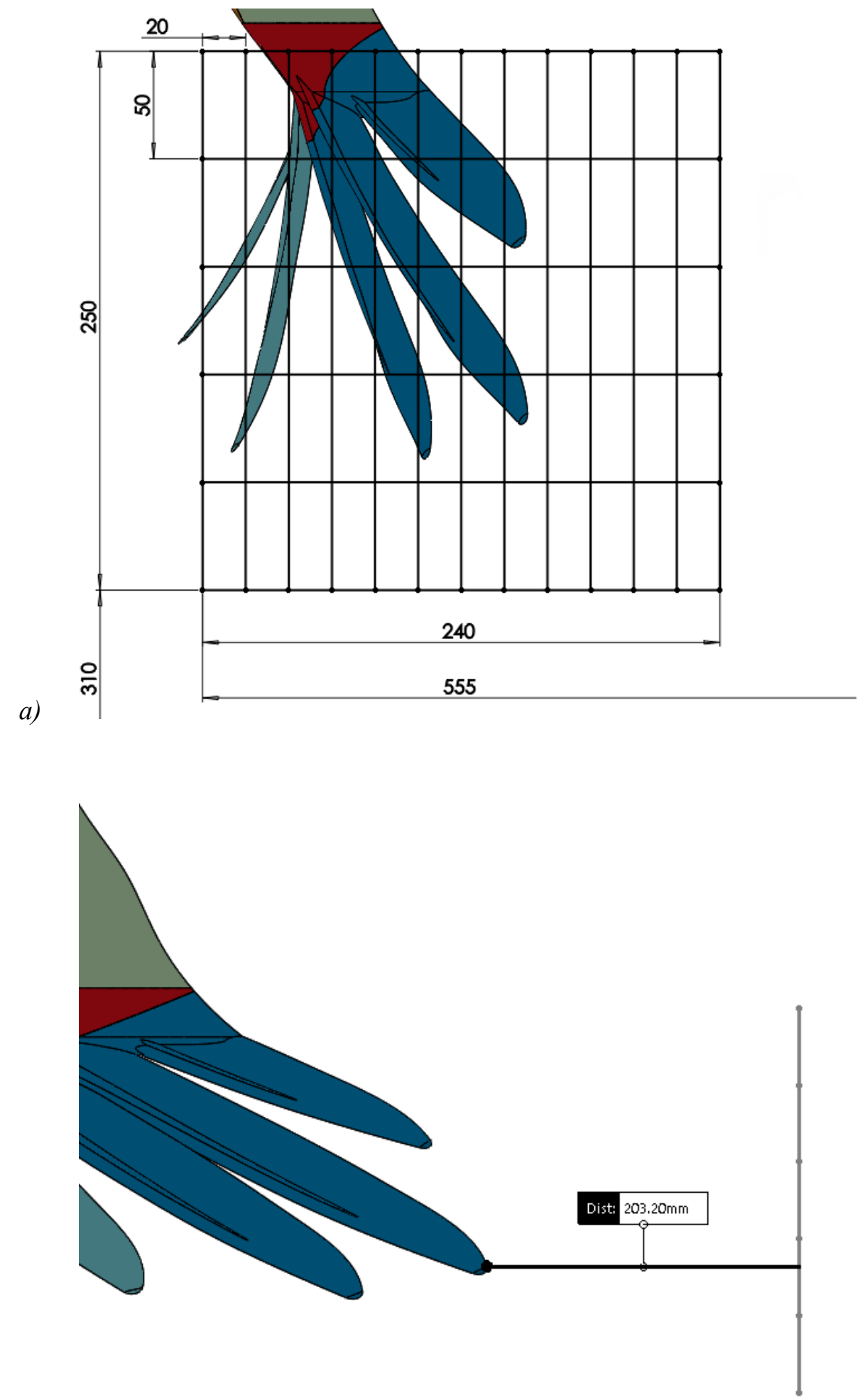

b)

Figure 32. Pressure rake measurement grid a) looking forward from downstream of the model and $\mathrm{b}$ ) side view. All dimensions are in $\mathrm{mm}$. 
The pressure data generated during testing were then post-processed in MATLAB to produce a plot of the ratio of the local flow velocity at the pressure ports to the free-stream velocity $\left(\mathrm{V}_{\mathrm{x}} / \mathrm{V}_{\mathrm{x} \infty}\right)$, which details the location and relative strength of each vortex structure. A copy of the post-processing MATLAB script is included in Appendix D for reference.

\subsection{Total Pressure Probe}

Subsequent pressure testing was carried out using the total pressure port of a five-hole probe, a standard instrument in aerodynamic testing. When properly calibrated, a five-hole probe is capable of resolving both the speed and direction of a flow in real time. In this case, while the pressure readings from all five pressure ports were recorded during testing, only the total pressure data collected by the center port was utilized during analysis due to the lack of an existing calibration matrix for the remaining ports. As such, only the flow velocity along the axis of the wind tunnel could be resolved during testing.

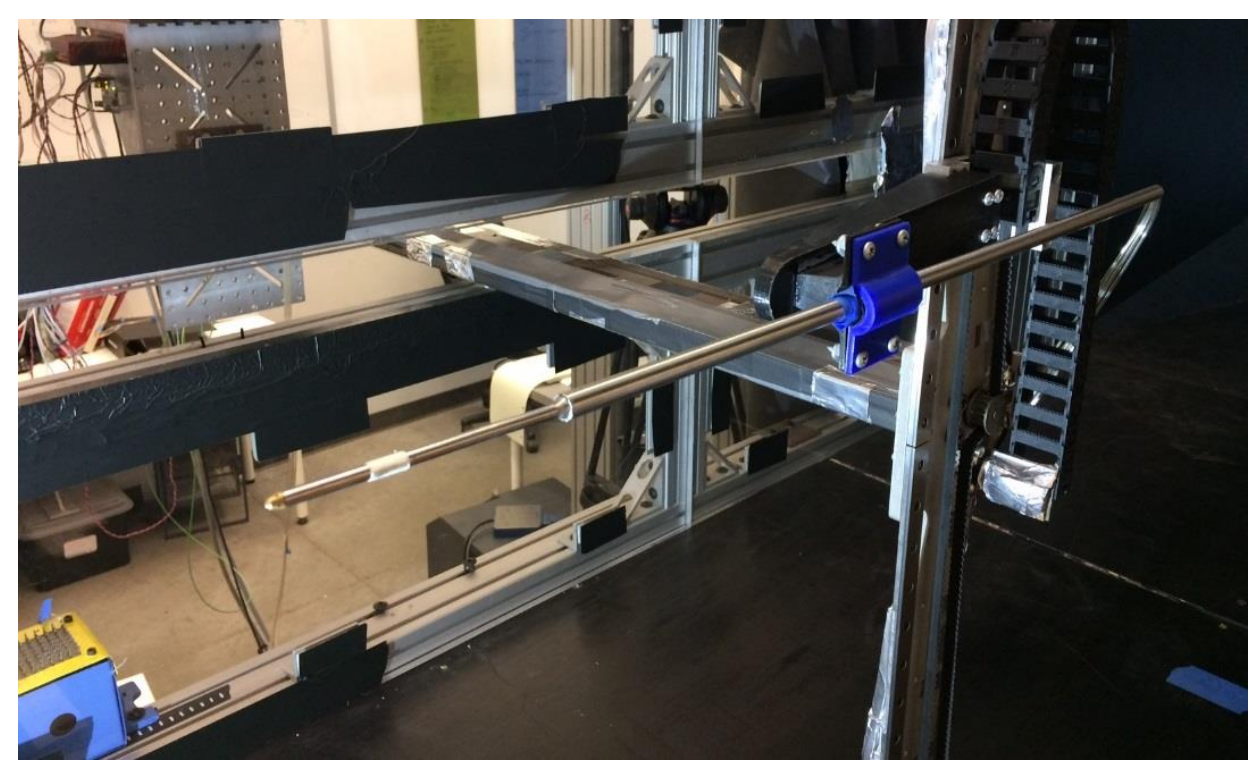

Figure 33. Total pressure probe setup. 
Pressure data was initially taken on a plane $0.2 \mathrm{~m}$ ( 0.59 reference chord lengths) downstream to allow for comparison to previous smoke visualization and pressure rake testing results. The size and shape of the grid was determined by analyzing vortex positions suggested by both visualization and pressure rake testing. The overall dimensions of the grid were slightly reduced from those of the pressure rake grid to $200 \mathrm{~mm}$ by $200 \mathrm{~mm}$, with measurements taken every $20 \mathrm{~mm}$ laterally and vertically. This led to a grid of 11 points by 11 points, or 121 sample points total. The position of the sampling grid in the wind tunnel is presented in Figure 34 . Similar to the grid used for the pressure rake, the dimensions of $310 \mathrm{~mm}$ and $555 \mathrm{~mm}$ refer to the distances to the test section floor and wall, respectively. 

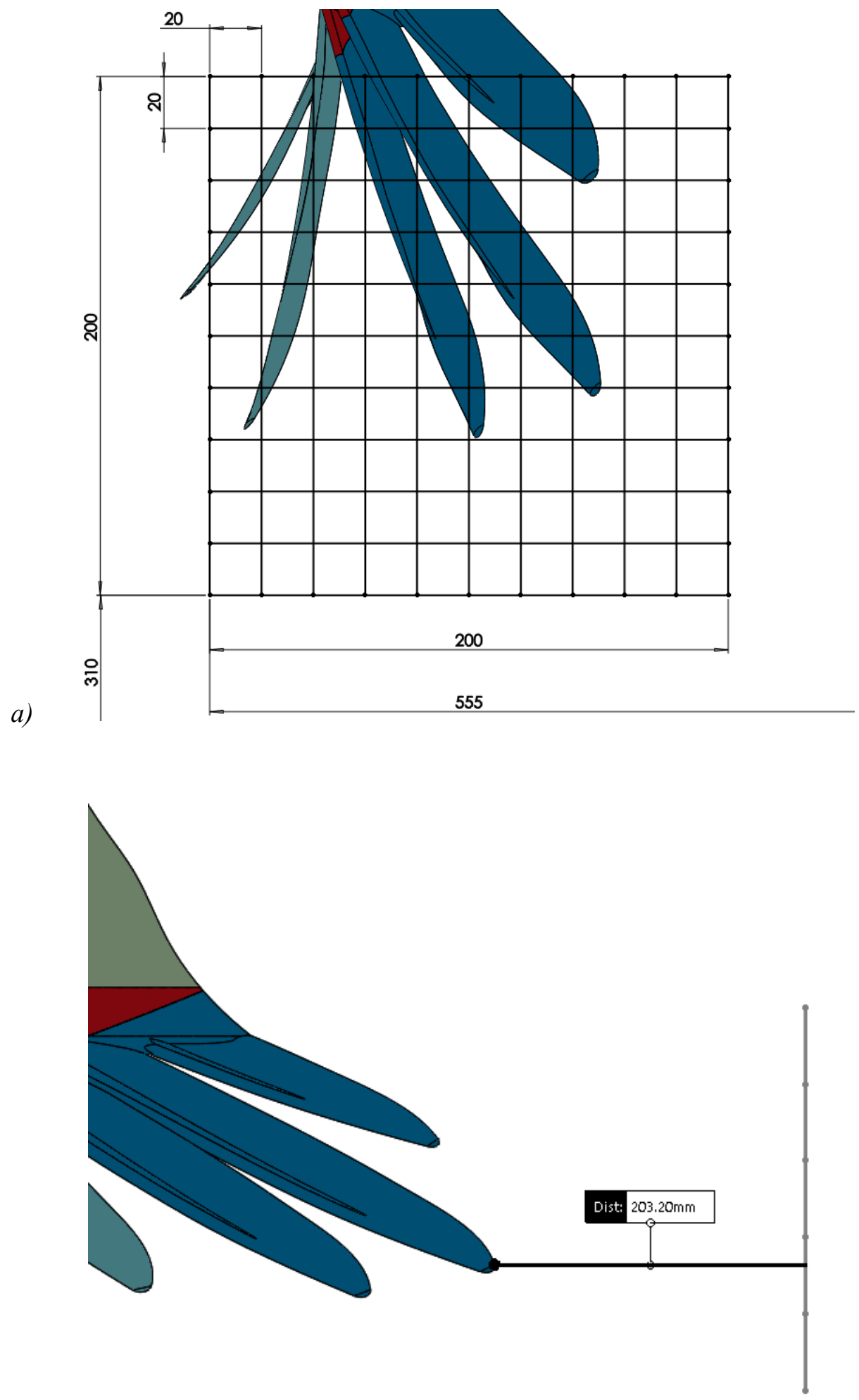

b)

Figure 34. First total pressure probe measurement grid a) looking forward from downstream of the model and b) side view. All dimensions are in $\mathrm{mm}$. 
After post-processing the data in MATLAB, the ratio of local velocity at the probe tip to the free-stream velocity $\left(\mathrm{V}_{\mathrm{x}} / \mathrm{V}_{\mathrm{x} \infty}\right)$ was plotted over the grid space to visualize the location and relative strength of each vortex. A sample of the post-processing MATLAB script is included in Appendix E for reference.

A second plane was then sampled at a distance of $0.1 \mathrm{~m}$ downstream of the fourth feather tip to investigate the evolution of the vortex structures over time. By nondimensionalizing using the chord of the scaled wing at the interface area between the wing body and primary feathers $(0.34 \mathrm{~m})$ for reference, a downstream distance of 0.29 chord lengths is obtained. The positioning of this grid was further refined by analyzing the results gathered during the first total pressure probe test. While this grid was also $200 \mathrm{~mm}$ by $200 \mathrm{~mm}$, samples were instead taken in increments of $10 \mathrm{~mm}$ both laterally and vertically. This resulted in a grid of 21 points by 21 points, or 441 sample points in total. While this higher resolution led to a significant increase in overall test time, the higher fidelity is immediately noticeable when comparing results between the two total pressure probe tests. After post-processing of the data, plots of the velocity ratio $\mathrm{V}_{\mathrm{x}} / \mathrm{V}_{\mathrm{x} \infty}$ were created in a similar fashion to those from the first total pressure probe test. Figure 35 details the location of the measurement grid. As before, the dimensions of $330 \mathrm{~mm}$ and $555 \mathrm{~mm}$ refer to the distances to the test section floor and wall, respectively. 

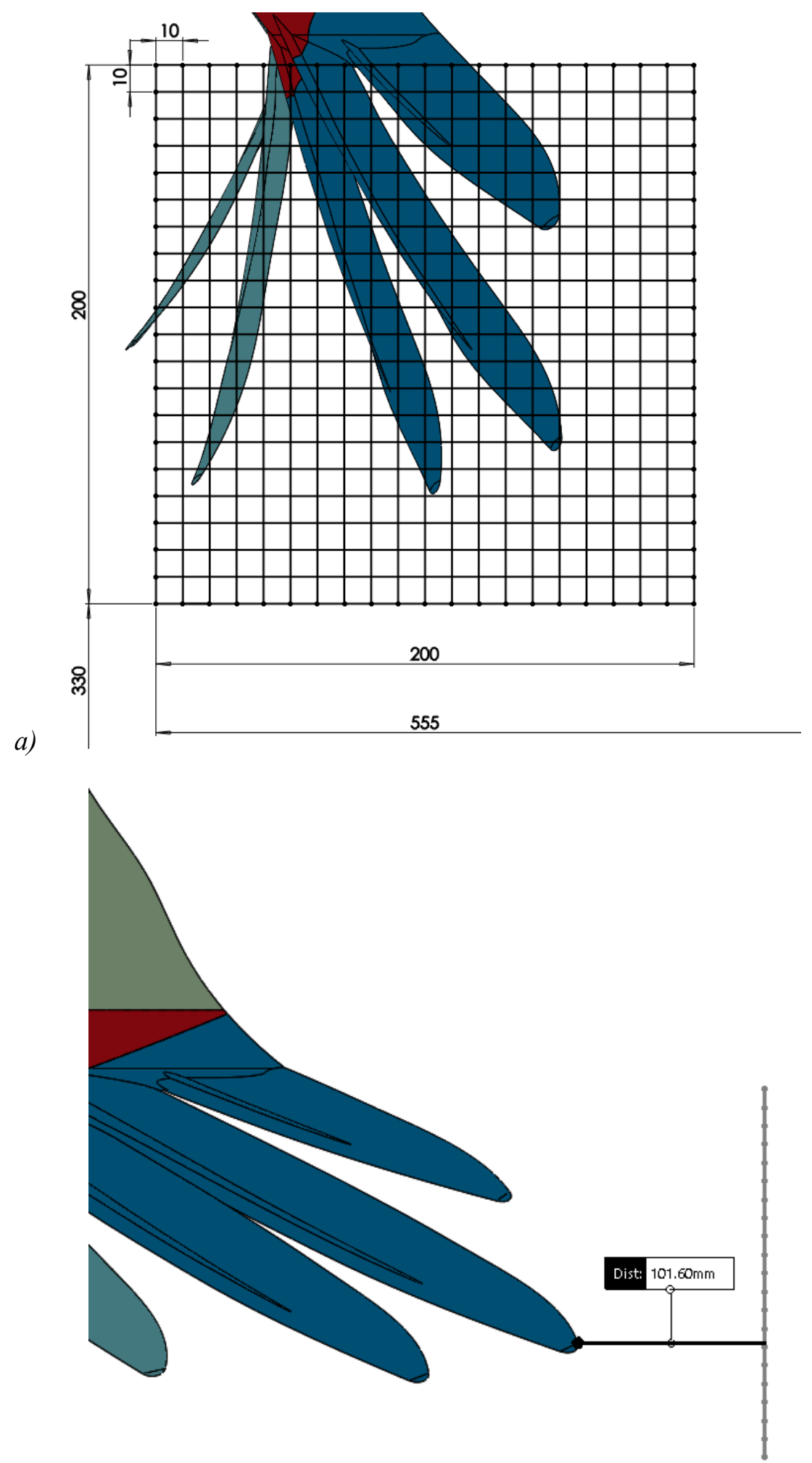

b)

Figure 35. Second total pressure probe measurement grid a) looking forward from downstream of the model and b) side view. All dimensions are in $\mathrm{mm}$. 


\section{EXPERIMENTAL PROCEDURE}

\subsection{Design of Experiments}

For a wind tunnel experiment to accurately replicate flight conditions, the Reynolds number and the Mach number must match those of the original conditions. ${ }^{28}$ These values are defined as follows:

$$
\begin{aligned}
R e & =\frac{\rho V L}{\mu} \\
M & =\frac{V}{a}
\end{aligned}
$$

where $\rho$ is the fluid density, $\mathrm{V}$ is the velocity of the fluid with respect to the object in question, $\mathrm{L}$ is the characteristic length, $\mu$ is the dynamic viscosity of the fluid and a is the local speed of sound. The Reynolds number describes the ratio of inertial forces to viscous forces in a fluid flow, whereas the Mach number is the ratio of the flow velocity to the local speed of sound. Both the Reynolds number and Mach number are dimensionless parameters.

As discussed previously, the gliding speed of a Brown Pelican is approximately $10 \mathrm{~m} / \mathrm{s}$. For convenience, the characteristic length has here been defined as the chord of the wing at the interface area between the wing body and primary feathers, which measures approximately $0.17 \mathrm{~m}$ on the $1: 1$ scale model. If $\rho$ and $\mu$ on a standard day at sea level are assumed to be $1.23 \mathrm{~kg} / \mathrm{m}^{3}$ and $1.73 \times 10^{-5} \mathrm{~N}-\mathrm{s} / \mathrm{m}^{2}$, respectively, the Reynolds number is on the order of $1.21 \times 10^{5}$. This places the air flow just below the transition point between laminar and turbulent flow. However, because the characteristic length was doubled as a result of concerns regarding the model's structural integrity, the flow velocity must be reduced by a factor of 2 to $5 \mathrm{~m} / \mathrm{s}$ to maintain Reynolds similarity. Due to the relatively small difference in flow velocity and the fact that the velocity is still well within the realm of incompressibility, the effect on Mach number is neglected. 
The final aerodynamic test model is capable of being position at three different angles of attack: $0^{\circ}, 5^{\circ}$ and $10^{\circ}$. Ultimately, all testing was performed at $0^{\circ}$ and $10^{\circ}$, though only results for the $10^{\circ}$ case are presented here. 


\section{RESULTS AND DISCUSSION}

\subsection{Airfoil Section Comparison}

Prior to beginning aerodynamic testing, several airfoil sections of the Brown Pelican wing were generated in Creo Parametric and compared to existing data from a detailed study of the Australian Pelican (Pelecanus conspicillatus) performed by Traill. ${ }^{9}$ In this study, two taxidermied Australian Pelican wings were 3D scanned: one in a thermal soaring position and one in a ground effect position. A collection of airfoil sections from each wing were then characterized using several standard airfoil measurements. The results of both characterizations will be considered here.

Because the outer surface of avian wings is primarily composed of a multitude of overlapping feather structures, airfoil sections of these wings are necessarily discontinuous. While the airfoil sections generated for the aerodynamic test model were designed to mimic the shape of the pelican's wing as accurately as possible, the airfoils presented in this discussion have been further simplified to allow comparison to standard NACA airfoils and other existing work. A visual comparison of the Brown Pelican airfoil geometry at eight spanwise locations is presented in Figure 36. 

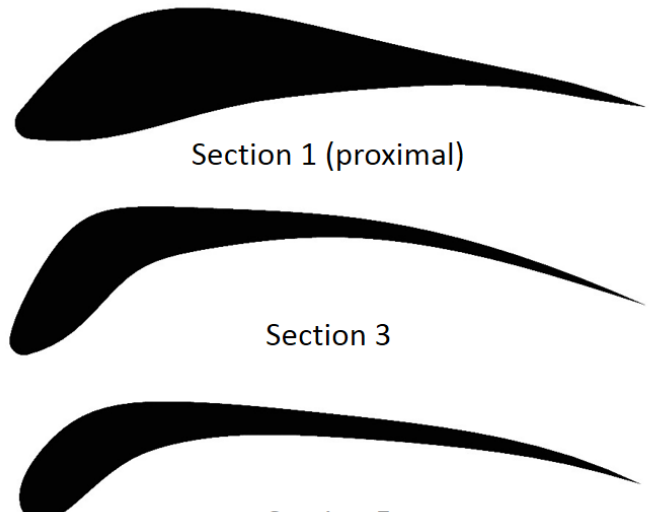

Section 5

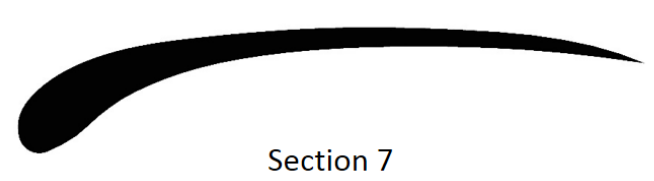

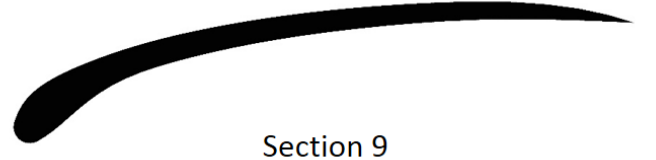
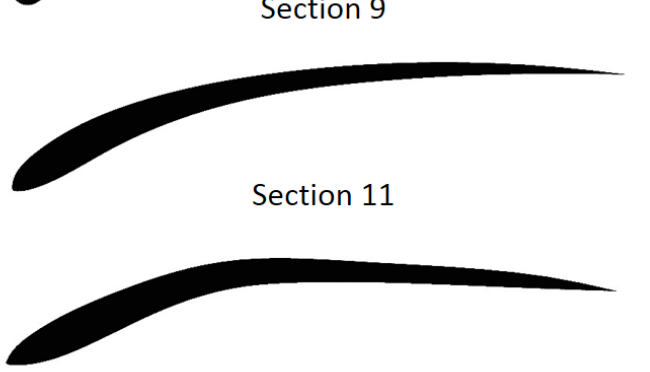

Section 13

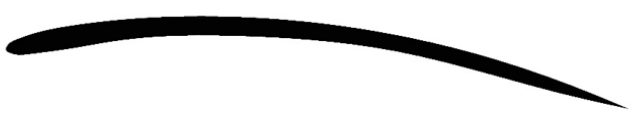

Section 15 (distal)

Figure 36. Simplified airfoil sections along the span of the Brown Pelican wing.

Sections 3 through 15 have been uniformly scaled for ease of viewing and comparison. The scaling factors with respect to the chord length of section 1 are presented in Table 1 for reference. Also shown is the angle of the chord line of each section with respect to the chord line of section 1, where positive angles represent a more nose-up configuration and negative angles represent a more nose-down configuration.

Table 1. Scaling factor and chord angle of each airfoil section with respect to that of section

1.

\begin{tabular}{|c|c|c|c|c|c|c|c|c|}
\hline Section & 1 & 3 & 5 & 7 & 9 & 11 & 13 & 15 \\
\hline Scaling Factor & 1.00 & 1.03 & 0.98 & 0.90 & 0.89 & 0.94 & 1.19 & 1.36 \\
\hline Angle (deg) & 0.00 & -2.13 & -1.17 & -6.10 & -8.69 & -8.43 & -4.64 & 7.43 \\
\hline
\end{tabular}


Table 2 outlines several common airfoil measurements gathered for each section. Note that all thickness, position of maximum thickness and camber values have been normalized by the airfoil chord length to obtain percentage values, a standard practice in airfoil characterization.

Table 2. Airfoil data for sections of the 3D scanned Brown Pelican wing.

\begin{tabular}{|c|c|c|c|c|c|c|}
\hline Section & $\begin{array}{c}\text { Distance } \\
\text { From Wing } \\
\text { Root }(\mathrm{mm})\end{array}$ & $\begin{array}{c}\text { Chord } \\
(\mathrm{mm})\end{array}$ & $\begin{array}{c}\text { Leading } \\
\text { Edge Radius } \\
(\mathrm{mm})\end{array}$ & $\begin{array}{c}\text { Maximum } \\
\text { Thickness }\end{array}$ & $\begin{array}{c}\text { Position of } \\
\text { Maximum } \\
\text { Thickness }\end{array}$ & Camber \\
\hline 1 & 0 & 235 & 6.0 & $17.8 \%$ & $22.4 \%$ & $10.1 \%$ \\
\hline 3 & 95 & 230 & 10.5 & $11.6 \%$ & $13.9 \%$ & $17.0 \%$ \\
\hline 5 & 191 & 245 & 8.0 & $10.3 \%$ & $9.0 \%$ & $14.0 \%$ \\
\hline 7 & 281 & 265 & 8.8 & $9.5 \%$ & $5.9 \%$ & $11.7 \%$ \\
\hline 9 & 347 & 267 & 6.9 & $6.7 \%$ & $5.5 \%$ & $9.9 \%$ \\
\hline 11 & 425 & 251 & 1.5 & $5.9 \%$ & $11.4 \%$ & $8.8 \%$ \\
\hline 13 & 509 & 197 & $\sim 0$ & $6.2 \%$ & $13.4 \%$ & $9.8 \%$ \\
\hline 15 & 606 & 173 & $\sim 0$ & $3.6 \%$ & $9.9 \%$ & $7.5 \%$ \\
\hline
\end{tabular}

Unsurprisingly, the airfoils presented here are markedly different than what might be expected on a modern aircraft. It is immediately apparent that the overall thickness of each airfoil is quite small, placing most within the classification of "thin airfoils" where the maximum airfoil thickness is much larger than the chord length. Despite these small thickness values, the leading edge radius of each airfoil is significantly larger than most modern airfoil sections. For example, the leading edge radius of airfoils contained in the NACA four digit series may be calculated using

$$
r=1.1019 t^{2}
$$

where $r$ is the leading edge radius and $t$ is the thickness. While the leading edge radius to thickness ratio of standard NACA airfoils are typically around 11\%, the largest leading edge radii presented here range anywhere from $31 \%$ to $39 \%$ of thickness for sections 3 through $9 .{ }^{6,8,9}$ 
Referencing the original 3D scan of the wing and published data regarding the skeletal structure of Brown Pelicans, it is estimated that the skeletal structure of the wing ends in the vicinity of section 11. This is evidenced by the larger leading edge radii and overall thickness of the inboard sections of the wing when compared to the outboard sections, which do not contain any of the wing's biological structure. As such, sections beyond section 11 are composed entirely of overlapping primary and secondary feathers, leading to a gradual decrease in thickness values from proximal to distal sections. As it is composed of feathers along the entire span of the wing, the trailing edge is clearly sharp in all sections.

The position of maximum thickness appears to gradually transition forward with an increase in span, though this trend reverses to some degree outboard of section 9. These results appear to correlate well with the Brown Pelican skeletal structure presented in Figure 7, suggesting that the forward transition in maximum thickness with increasing span is due to the presence of the radius, ulna and metacarpus within the wing structure, as well as the related musculature. In addition, the leading edge of the wing is the proximity of sections 1 through 7 is primarily composed of tendons with the skeletal structure of the wing housed further aft, which necessarily pushes the position of maximum thickness aft as well. Outboard of section 9, the position of maximum thickness transitions rearward, corresponding with the location of the metacarpal bones.

The values for the camber of each airfoil section presented in Table 2, while larger than most modern airfoils, are what might be intuitively expected for an avian wing. However, this intuition does not fully explain the aerodynamic purpose behind the highly cambered inboard wing and relatively planar outboard wing. While this question has not been definitively answered in published literature, studies by Tucker ${ }^{24}$ and Rosen $^{25}$ suggest that the majority of lift required for avian flight is primarily generated by the inboard section of the wing. Neither the Harris' hawk nor the jackdaw investigated in these two studies fully extended their wings during gliding flight, even at the lowest speeds. In fact, Rosen concludes that there is a linear relationship between wingspan 
and airspeed in the case of the jackdaw (and other avian species), with the wingspan decreasing for an increase in airspeed. This is somewhat counterintuitive when considering induced drag, where a larger wing aspect ratio results in more efficient drag reduction. However, it may be that by producing the majority of the necessary lift using the inboard section of the wing, the outboard section is therefore free to be used for other purposes, such as twisting and warping for aerodynamic control, flapping, etc. Additional studies using live birds will likely need to be conducted in the future to fully characterize the lift generation capabilities of various avian wings.

The following figures present a comparison between the Brown Pelican wing sections and wing sections of the Australian Pelican generated by Traill. ${ }^{9}$ Figures 37 and 38 compare the Brown Pelican sections to an Australian Pelican wing in a thermal soaring configuration, whereas Figures 39 and 40 compare the Brown Pelican sections to an Australian Pelican wing in a ground effect configuration. The section number noted in Traill's work refers to the distance from the wing root in $20 \mathrm{~mm}$ increments (e.g. section 15 is $300 \mathrm{~mm}$ from the wing root). 

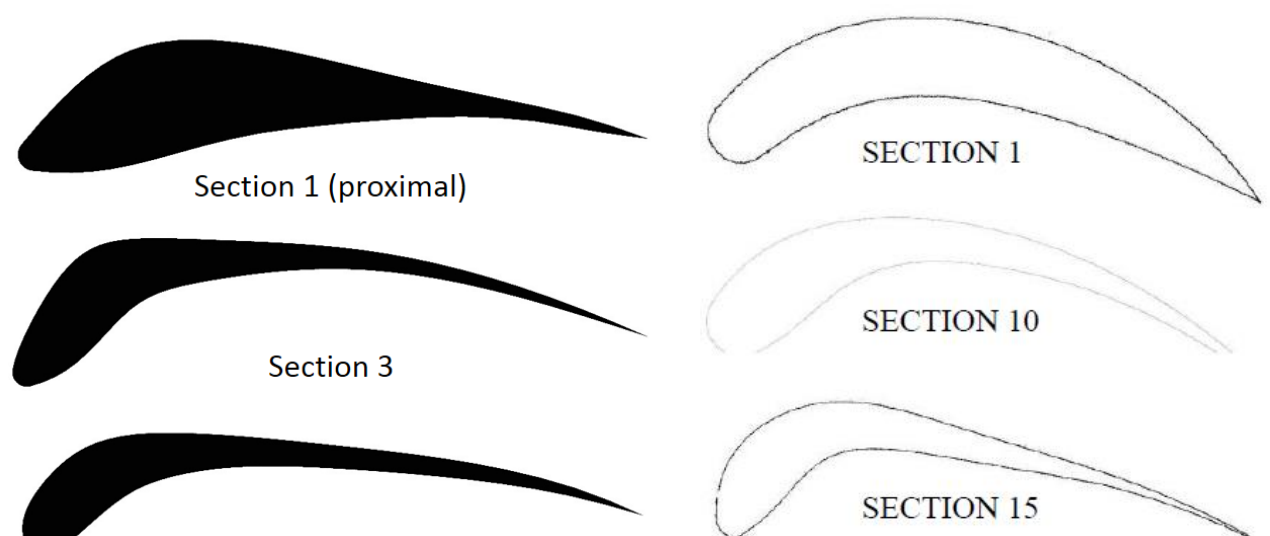

Section 5

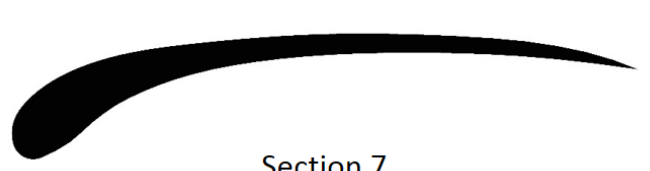

Section 7

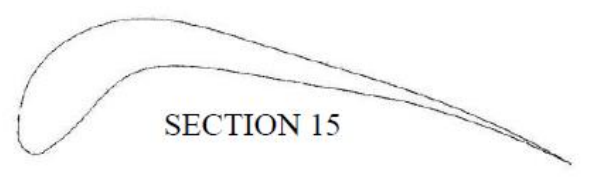

\section{SECTION 10}
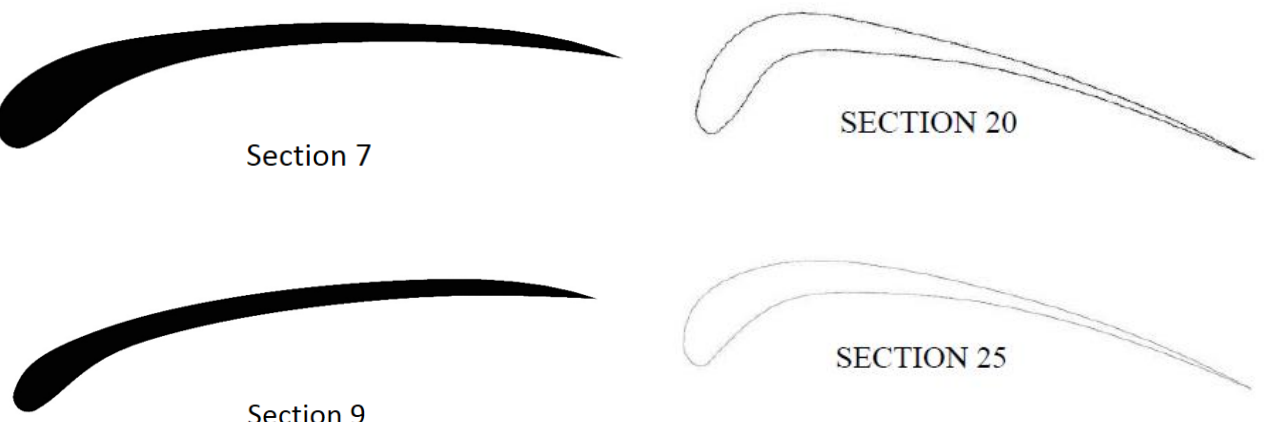

Section 9
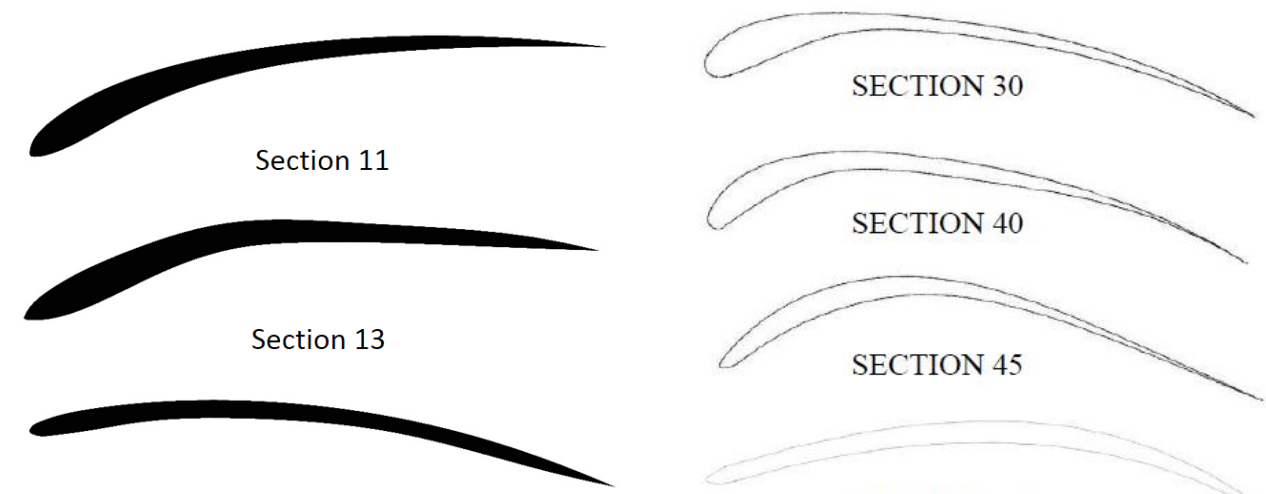

Section 15 (distal)

SECTION 50

Brown Pelican

Pelecanus occidentalis

Australian Pelican

Pelecanus conspicillatus

Figure 37. Simplified airfoil sections from the wing of a Brown Pelican compared to sections from an Australian Pelican in a thermal soaring configuration generated by Traill. ${ }^{9}$ For visual reference only, not to scale. 


\begin{tabular}{|c|c|c|c|c|c|c|}
\hline Section & $\begin{array}{c}\text { Distance } \\
\text { From Wing } \\
\text { Root }(\mathrm{mm})\end{array}$ & $\begin{array}{c}\text { Chord } \\
(\mathrm{mm})\end{array}$ & $\begin{array}{c}\text { Leading } \\
\text { Edge Radius } \\
(\mathrm{mm})\end{array}$ & Thickness & $\begin{array}{c}\text { Position of } \\
\text { Max. } \\
\text { Thickness }\end{array}$ & Camber \\
\hline 1 & 0 & 235 & 6.0 & $17.8 \%$ & $22.4 \%$ & $10.1 \%$ \\
\hline 3 & 95 & 230 & 10.5 & $11.6 \%$ & $13.9 \%$ & $17.0 \%$ \\
\hline 5 & 191 & 245 & 8.0 & $10.3 \%$ & $9.0 \%$ & $14.0 \%$ \\
\hline 7 & 281 & 265 & 8.8 & $9.5 \%$ & $5.9 \%$ & $11.7 \%$ \\
\hline 9 & 347 & 267 & 6.9 & $6.7 \%$ & $5.5 \%$ & $9.9 \%$ \\
\hline 11 & 425 & 251 & 1.5 & $5.9 \%$ & $11.4 \%$ & $8.8 \%$ \\
\hline 13 & 509 & 197 & $\sim 0$ & $6.2 \%$ & $13.4 \%$ & $9.8 \%$ \\
\hline 15 & 606 & 173 & $\sim 0$ & $3.6 \%$ & $9.9 \%$ & $7.5 \%$ \\
\hline
\end{tabular}

Brown Pelican

Pelecanus occidentalis

\begin{tabular}{|c|c|c|c|c|c|}
\hline Section & Chord & LE Radius & Thickness & $\begin{array}{l}\text { Position Max } \\
\text { Thickness }\end{array}$ & Camber \\
\hline \hline 1 & $202 \mathrm{~mm}$ & $11.8 \mathrm{~mm}$ & $14.0 \%$ & $20.7 \%$ & $20.5 \%$ \\
\hline 5 & $290 \mathrm{~mm}$ & $12.0 \mathrm{~mm}$ & $13.0 \%$ & $18.2 \%$ & $16.8 \%$ \\
\hline 10 & $276 \mathrm{~mm}$ & $17.3 \mathrm{~mm}$ & $13.9 \%$ & $14.2 \%$ & $21.9 \%$ \\
\hline 15 & $276 \mathrm{~mm}$ & $10.3 \mathrm{~mm}$ & $12.1 \%$ & $12.6 \%$ & $20.7 \%$ \\
\hline 20 & $283 \mathrm{~mm}$ & $8.8 \mathrm{~mm}$ & $8.4 \%$ & $11.6 \%$ & $19.0 \%$ \\
\hline 25 & $291 \mathrm{~mm}$ & $8.3 \mathrm{~mm}$ & $8.6 \%$ & $9.1 \%$ & $16.7 \%$ \\
\hline 30 & $300 \mathrm{~mm}$ & $8.5 \mathrm{~mm}$ & $7.3 \%$ & $9.1 \%$ & $11.9 \%$ \\
\hline 35 & $256 \mathrm{~mm}$ & $5.2 \mathrm{~mm}$ & $5.9 \%$ & $9.1 \%$ & $14.1 \%$ \\
\hline 40 & $225 \mathrm{~mm}$ & $\sim 2.0 \mathrm{~mm}$ & $4.2 \%$ & $19.2 \%$ & $17.0 \%$ \\
\hline 45 & $178 \mathrm{~mm}$ & $\sim 0 \mathrm{~mm}$ & - & - & $18.2 \%$ \\
\hline 50 & $160 \mathrm{~mm}$ & $\sim 0 \mathrm{~mm}$ & - & - & $12.4 \%$ \\
\hline
\end{tabular}

Australian Pelican

Pelecanus conspicillatus

Figure 38. Comparison of airfoil characterization data between the Brown Pelican wing and Australian Pelican wing in a thermal soaring configuration generated by Traill. ${ }^{9}$ 

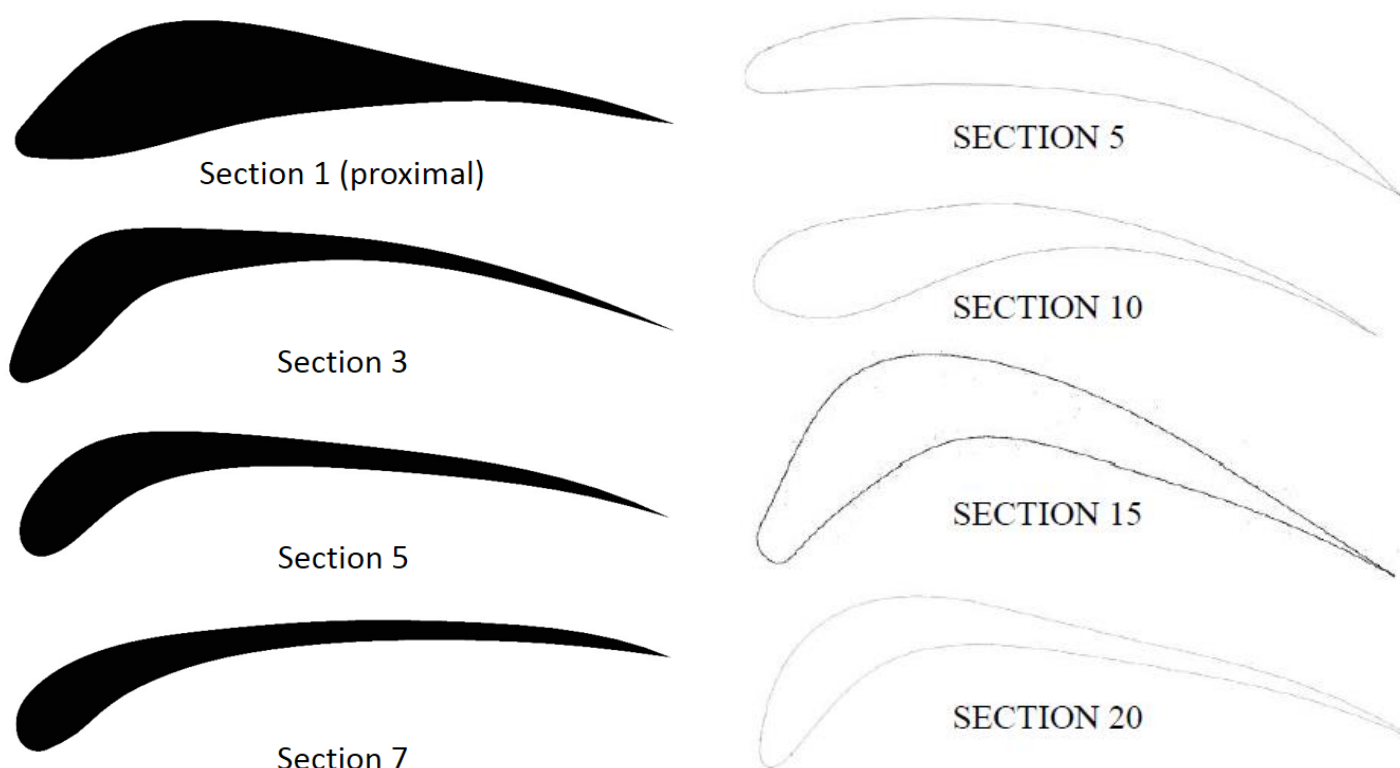

Section 7
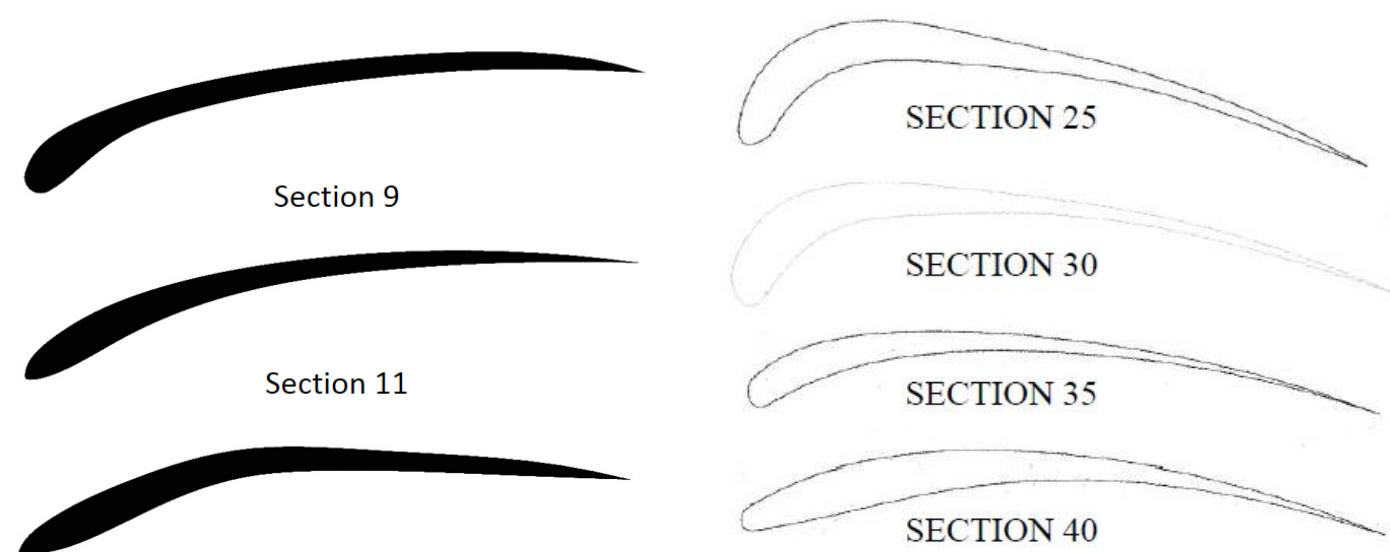

Section 13

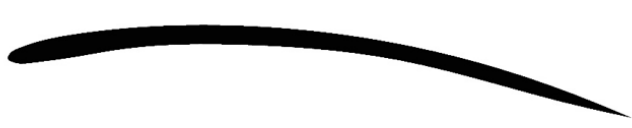

Section 15 (distal)

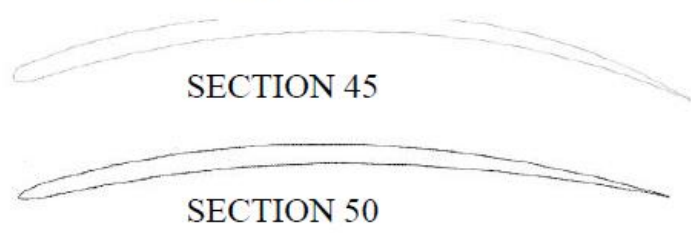

Brown Pelican

Pelecanus occidentalis

Australian Pelican

Pelecanus conspicillatus

Figure 39. Simplified airfoil sections from the wing of a Brown Pelican compared to sections from an Australian Pelican in a ground effect configuration generated by Traill. ${ }^{9}$ For visual reference only, not to scale. 


\begin{tabular}{|c|c|c|c|c|c|c|}
\hline Section & $\begin{array}{c}\text { Distance } \\
\text { From Wing } \\
\text { Root }(\mathrm{mm})\end{array}$ & $\begin{array}{c}\text { Chord } \\
(\mathrm{mm})\end{array}$ & $\begin{array}{c}\text { Leading } \\
\text { Edge Radius } \\
(\mathrm{mm})\end{array}$ & Thickness & $\begin{array}{c}\text { Position of } \\
\text { Max. } \\
\text { Thickness }\end{array}$ & Camber \\
\hline 1 & 0 & 235 & 6.0 & $17.8 \%$ & $22.4 \%$ & $10.1 \%$ \\
\hline 3 & 95 & 230 & 10.5 & $11.6 \%$ & $13.9 \%$ & $17.0 \%$ \\
\hline 5 & 191 & 245 & 8.0 & $10.3 \%$ & $9.0 \%$ & $14.0 \%$ \\
\hline 7 & 281 & 265 & 8.8 & $9.5 \%$ & $5.9 \%$ & $11.7 \%$ \\
\hline 9 & 347 & 267 & 6.9 & $6.7 \%$ & $5.5 \%$ & $9.9 \%$ \\
\hline 11 & 425 & 251 & 1.5 & $5.9 \%$ & $11.4 \%$ & $8.8 \%$ \\
\hline 13 & 509 & 197 & $\sim 0$ & $6.2 \%$ & $13.4 \%$ & $9.8 \%$ \\
\hline 15 & 606 & 173 & $\sim 0$ & $3.6 \%$ & $9.9 \%$ & $7.5 \%$ \\
\hline
\end{tabular}

Brown Pelican

Pelecanus occidentalis

\begin{tabular}{|c|c|c|c|c|c|}
\hline Section & Chord & LE Radius & Thickness & $\begin{array}{c}\text { Position Max } \\
\text { Thickness }\end{array}$ & Camber \\
\hline \hline 5 & $217 \mathrm{~mm}$ & $6.1 \mathrm{~mm}$ & $10.1 \%$ & $24.5 \%$ & $21.4 \%$ \\
\hline 10 & $270 \mathrm{~mm}$ & $12.5 \mathrm{~mm}$ & $15.0 \%$ & $22.1 \%$ & $22.3 \%$ \\
\hline 15 & $283 \mathrm{~mm}$ & $9.3 \mathrm{~mm}$ & $16.0 \%$ & $19.1 \%$ & $25.4 \%$ \\
\hline 20 & $272 \mathrm{~mm}$ & $7.0 \mathrm{~mm}$ & $10.2 \%$ & $14.7 \%$ & $21.2 \%$ \\
\hline 25 & $294 \mathrm{~mm}$ & $7.2 \mathrm{~mm}$ & $7.3 \%$ & $10.1 \%$ & $17.2 \%$ \\
\hline 30 & $314 \mathrm{~mm}$ & $10.5 \mathrm{~mm}$ & $7.9 \%$ & $7.9 \%$ & $14.4 \%$ \\
\hline 35 & $271 \mathrm{~mm}$ & $6.4 \mathrm{~mm}$ & $5.6 \%$ & $12.0 \%$ & $9.3 \%$ \\
\hline 40 & $227 \mathrm{~mm}$ & $4.1 \mathrm{~mm}$ & $7.0 \%$ & $18.5 \%$ & $9.6 \%$ \\
\hline 45 & $175 \mathrm{~mm}$ & $\sim 0 \mathrm{~mm}$ & - & - & $9.5 \%$ \\
\hline 50 & $128 \mathrm{~mm}$ & $\sim 0 \mathrm{~mm}$ & - & - & $6.7 \%$ \\
\hline
\end{tabular}

Australian Pelican

Pelecanus conspicillatus

Figure 40. Comparison of airfoil characterization data between the Brown Pelican wing and Australian Pelican wing in a ground effect configuration generated by Traill. ${ }^{9}$ 
While the results of the Brown Pelican airfoil characterization correlate well to those of the Australian Pelican in terms of general wing geometry and trends in airfoil characteristics, the Brown Pelican wing appears to be much more similar in form to the Australian Pelican wing preserved in a thermal soaring configuration. This suggests that the Brown Pelican wing has also been preserved in such a configuration. Despite good overall agreement with Traill's work, several discrepancies are apparent. Beyond section 9, the leading edge of the Brown Pelican wing is composed entirely of primary feathers and the leading edge radius becomes sharp. However, the leading edge radius on both Australian Pelican wings was found to be unexpectedly large for many of the distal sections. Traill reasonably remarks that this is unusual and inconsistent with existing literature describing avian wings. Additionally, the Australian Pelican wings are significantly more cambered than that of the Brown Pelican, especially for distal sections. This is particularly apparent near the wing root and mid-wing in both Australian Pelican configurations. Additional research will need to be conducted to confirm whether these discrepancies are due to differences in the taxidermizing process, differences between the two species or perhaps simply features specific to each individual bird.

The Australian Pelican wings also appear to have a slightly more nose-up angle of attack. While the pitch of the wing will not affect the overall geometry of the scanned wings, these differences may skew the results of subsequent aerodynamic analysis. However, determining a precise angle of attack is challenging under the best of circumstances. The method suggested by Traill, which involves determining the angle of attack of the root by referencing pictures taken during wing upstrokes, was used in this case. However, this method still leaves some degree of uncertainty and is difficult to implement using pictures where no horizontal reference exists (assuming the pelican is traveling in a horizontal direction to begin with).

While this study provides characterization of an example Brown Pelican wing, it should be noted that the geometry presented here may not be representative of all Brown Pelicans. Biological 
differences between specimens or inaccuracies introduced during the taxidermizing process are likely to introduce some variability to wing geometry. As such, additional studies will need to be carried out to obtain a more detailed view of the aerodynamics of Brown Pelican wings, as well as an "average" wing shape.

Finally, a simple study was conducted to provide a quantitative measure of the various angles of attack of each primary feather. Feathers were sectioned at their respective mid-spans and compared to the chord line of wing section 1. It can be seen in Table 3 that, while the mid-span of the most forward feathers have a slight negative angle of attack, the mid-span of the rearmost are instead at a positive angle of attack.

Table 3. Angle of attack of each primary feather measured at the mid-span with reference to the chord of wing section 1.

\begin{tabular}{|c|c|c|c|c|c|}
\hline Feather & 1 & 2 & 3 & 4 & 5 \\
\hline Angle of Attack (deg) & -5.56 & -5.86 & -0.07 & 5.90 & 20.63 \\
\hline
\end{tabular}

\subsection{Flow Visualization Results}

Flow visualization results were obtained by using the Rosco fog machine in conjunction with the Laser Modules line laser at a flow velocity of $5 \mathrm{~m} / \mathrm{s}$. Figure 41 presents a still frame taken from a video footage with the wing at an angle of attack of $10^{\circ}$ outlining the size and location of vortices observed at $0.2 \mathrm{~m}$ ( 0.59 reference chord lengths) downstream of the tip of the fourth feather. The three vortices visible are created by the $1^{\text {st }}, 2^{\text {nd }}$ and $4^{\text {th }}$ primary feathers and are labeled 1, 2 and 4, respectively. A series of more informative images can be found in Figure 42. Each of these three vortices held a relatively fixed position in space and was found to persist throughout the duration of the experiment. An additional vortex (vortex 3) suggested by subsequent pressure testing is also presented in Figure 41, though both the strength and positioning of this vortex 
structure appeared to be somewhat inconsistent during the visualization process and it is noted here for reference only. It should be noted that the brightness and contrast of Figures 41 through 43 have been artificially increased using Adobe Photoshop to enhance the visibility of flow phenomena.

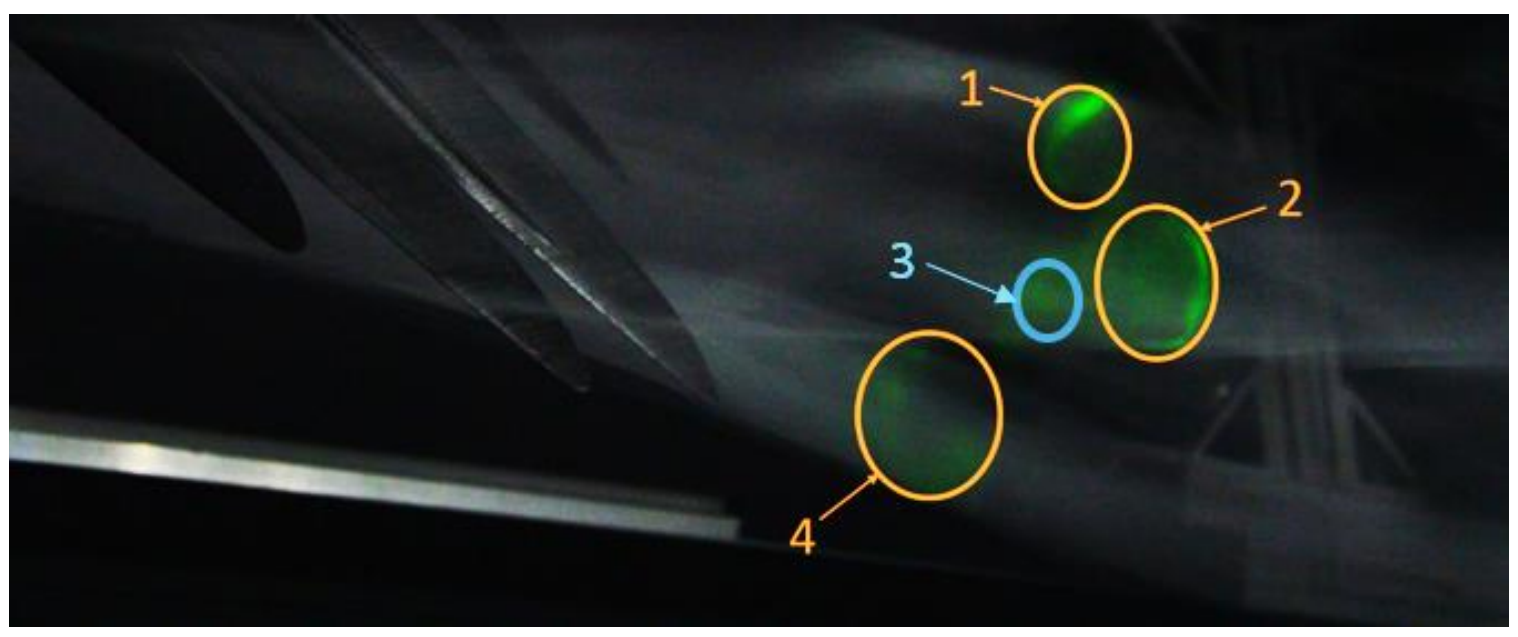

Figure 41. Still frame detailing relative size and location of vortices downstream of the test model. Flow velocity $5 \mathrm{~m} / \mathrm{s}$, angle of attack $10^{\circ}$. 

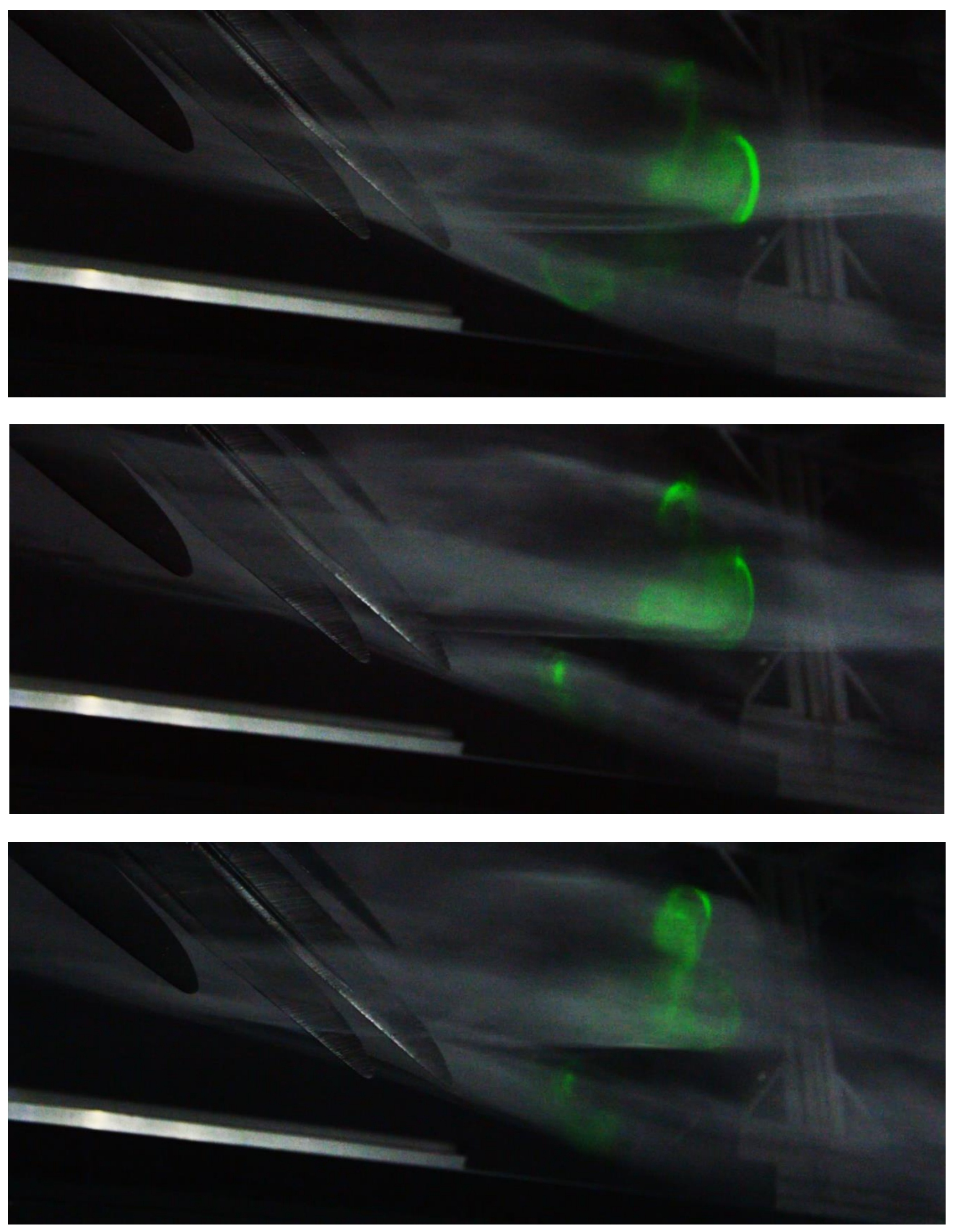

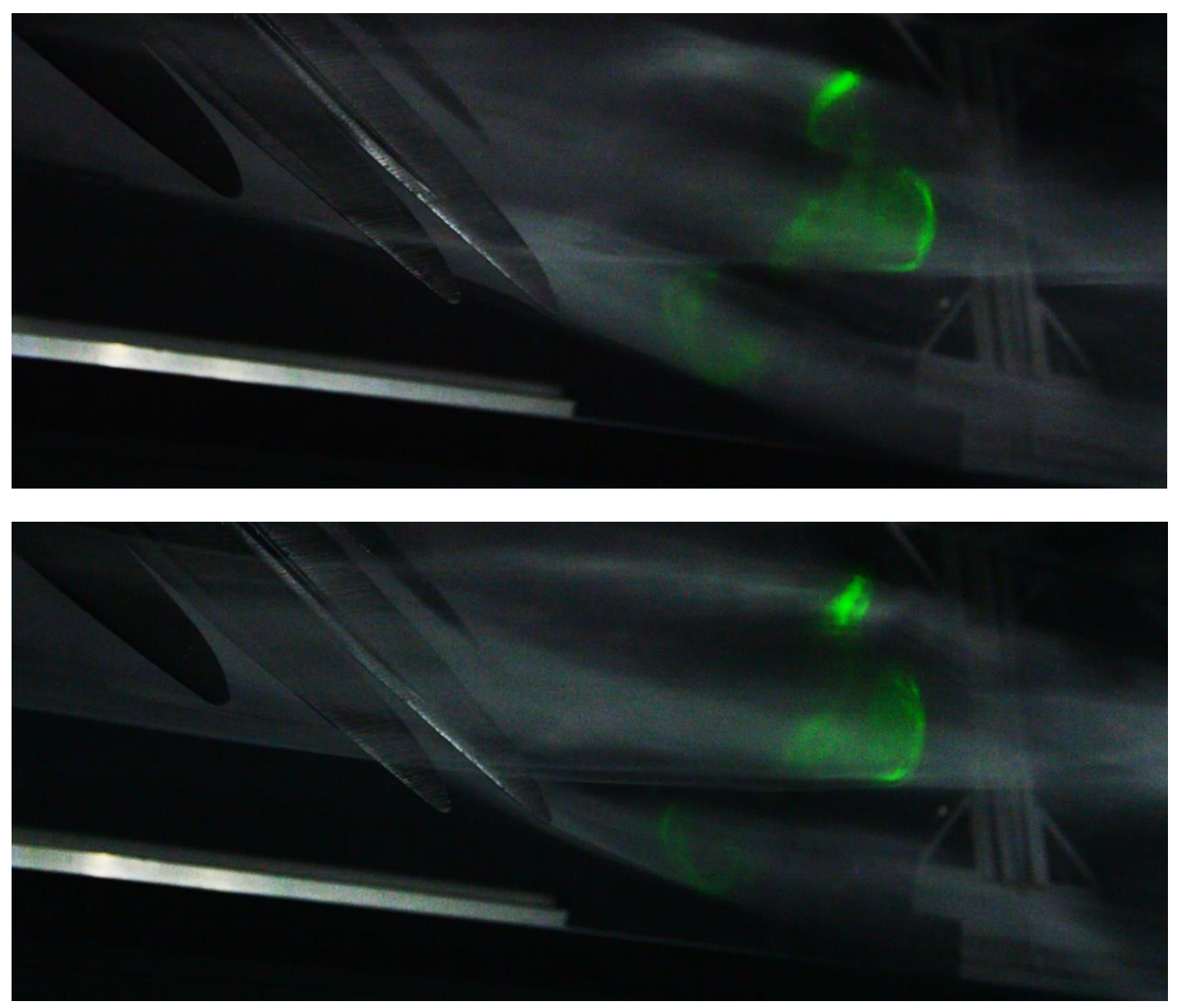

Figure 42. Collection of still frames showing vortex development from the $1^{\text {st }}, 2^{\text {nd }}$ and $4^{\text {th }}$ primary feathers. Flow velocity $5 \mathrm{~m} / \mathrm{s}$, angle of attack $10^{\circ}$.

Also visible during testing was a pronounced inboard deflection of the flow above the upper surfaces of the primary feathers. The flow on the underside of the primary feathers was also noted to deflect slightly outboard. These effects are thought to be indicative of the formation of a large-scale pressure gradient created by the region of relatively high pressure below the wing and low pressure above the wing. In this case, the low flow velocity allows the effects of this gradient to be visible in the vicinity of the wing itself as opposed to further downstream. Additionally, each primary feather is likely to be deflecting the flow either inboard or outboard based on the relative 
angle between the plane of the wing and the feather. Figure 43 details the flow deflections above and below the wing.
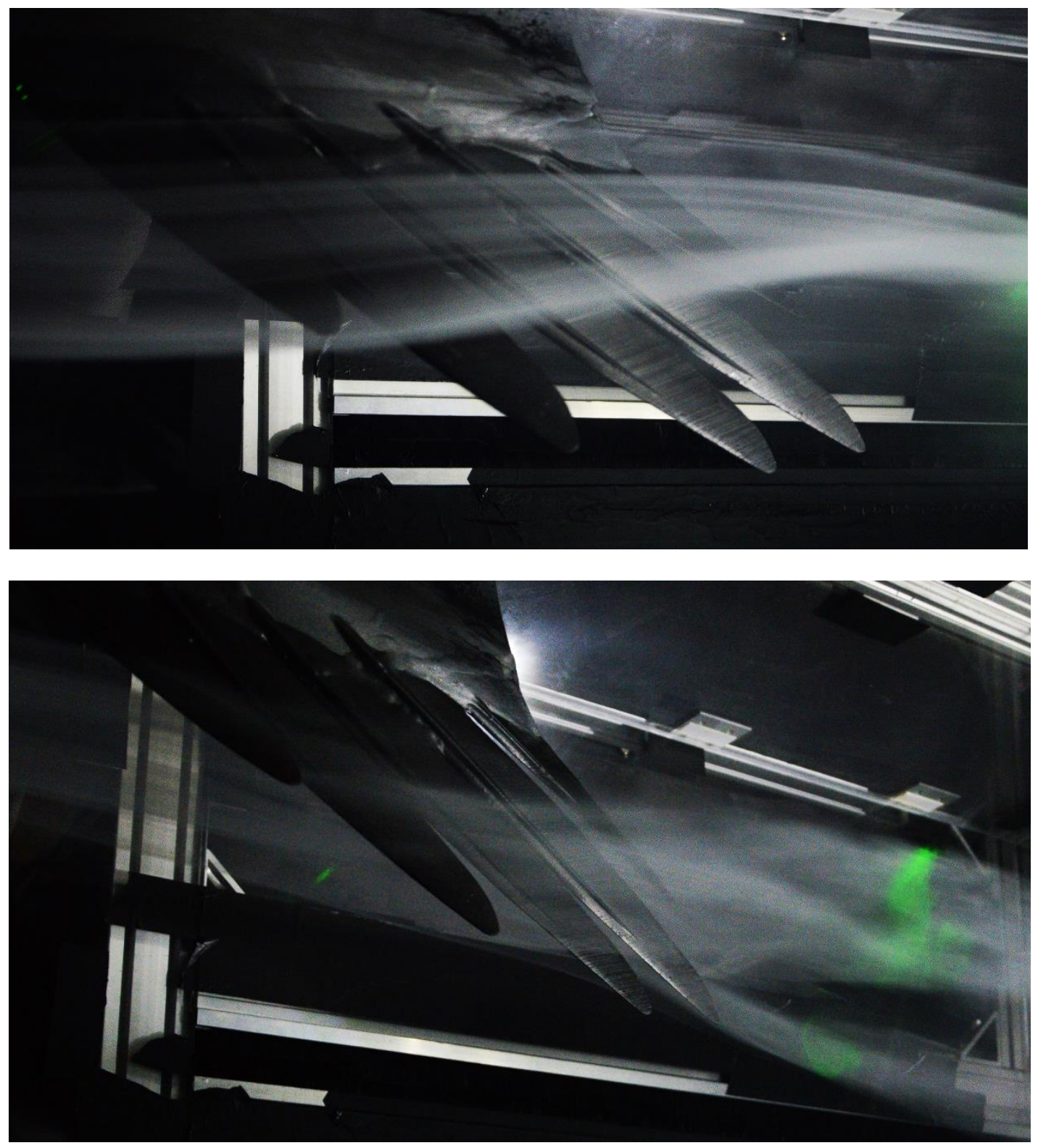

Figure 43. Flow deflection around the primary feathers. Side view.

Visualization results obtained at $0^{\circ}$ angle of attack were similar to those outlined above, though the vortices were somewhat less pronounced. 


\subsection{Pressure Rake Results}

To more accurately characterize the position and relative strength of the vortex structures generated by the primary feathers, a total pressure rake was used to sample total pressure values on a two-dimensional plane $0.2 \mathrm{~m}$ downstream of the model. This test was conducted with the wing at $10^{\circ}$ angle of attack and a flow speed of $5 \mathrm{~m} / \mathrm{s}$. The recorded pressure data was then post-processed in MATLAB. The resulting distribution of the velocity ratio $V_{\mathrm{x}} / \mathrm{V}_{\mathrm{x} \infty}$, or the ratio of the local velocity at the pressure rake to free stream velocity, was plotted over the grid space. 

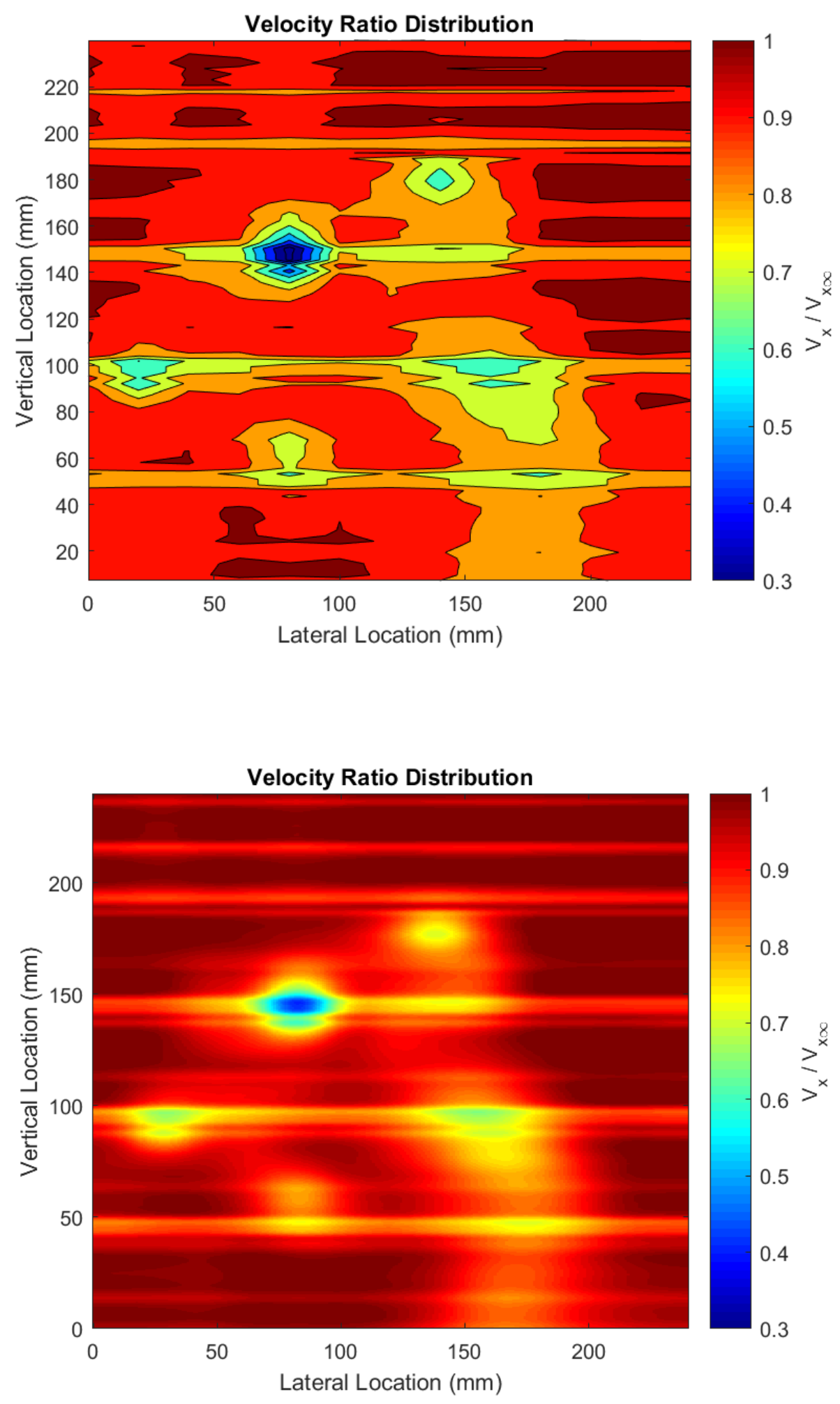

Figure 44. Plot of the velocity ratio $V_{x} / V_{x \infty}$ generated using a pressure rake at a distance of $0.2 \mathrm{~m}$ downstream of the model and a flow velocity of $5 \mathrm{~m} / \mathrm{s}$. 

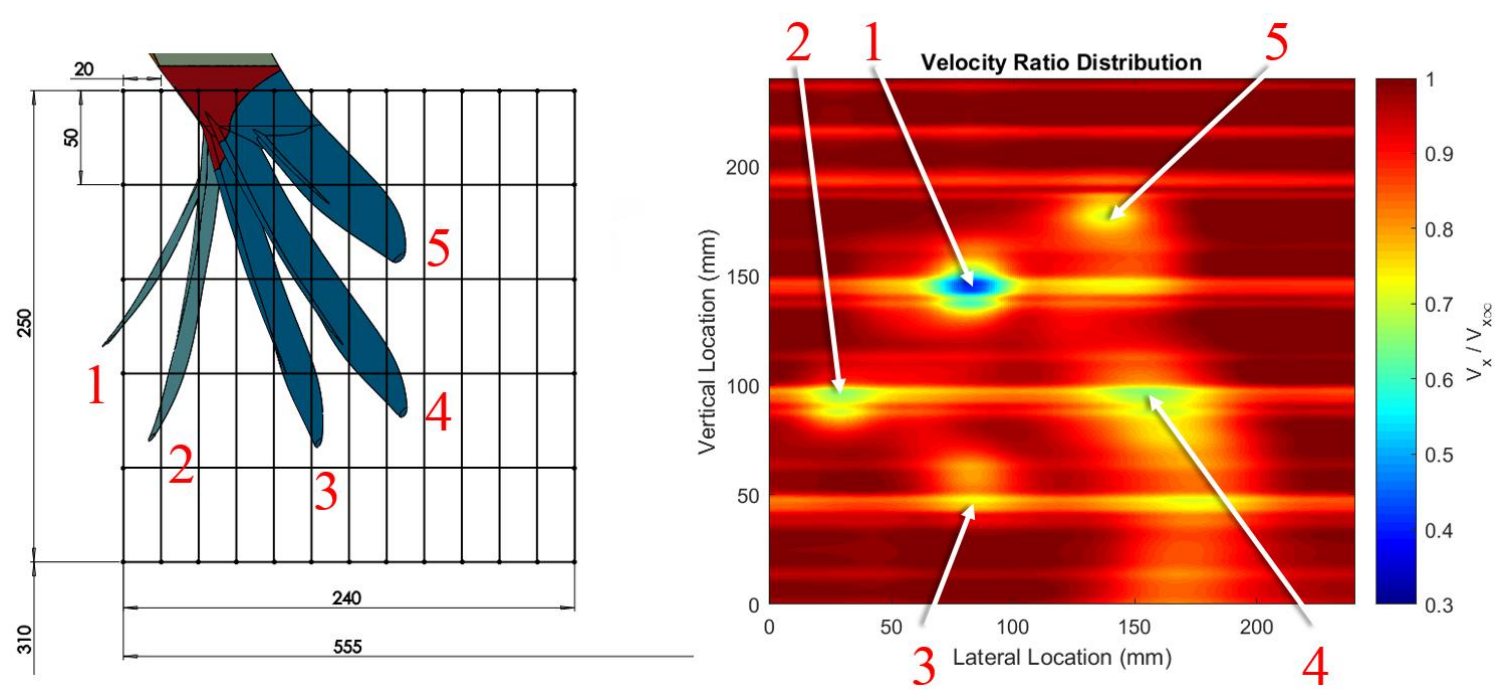

Figure 45. Comparison of the $\mathrm{CAD}$ geometry to data generated during pressure rake testing with the sampling plane located $0.2 \mathrm{~m}$ downstream of the model. Each vortex structure and its generating feather are matched by number. All dimensions are in $\mathrm{mm}$.

Post-processing the data collected by the pressure rake presented significant difficulties due to unexpectedly low pressure readings at both the upper and lower ends of the rake, as well as a blocked pressure port near the center of the rake. Figure 44 is the result of an extensive data postprocessing and refinement process and was generated by replacing anomalous data with an average of the surrounding data. Even after refinement, large-scale striations running from left to right are immediately apparent. These striations are artifacts of the artificially low pressure readings generated at each end of the rake and, in general, do not represent physical flow phenomena.

Despite the difficulties encountered, several vortex structures generated by the primary feathers are visible in Figure 44. While the vortices created by feathers 1, 2 and 3 are clearly formed, the remaining vortex structures are less distinct. This effect is likely to have been caused by vortices wandering over time in an unsteady flow or stalled flow over the primary feathers due to a relatively high angle of attack. 
The positioning of vortices 3,4 and 5 correlates very well with the positioning of their respective feathers. However, the positioning of vortices 1 and 2 deviates significantly from the position of each generating feather, with both vortices being pulled right and upwards. This result should in fact be expected based on the results of earlier visualization testing, where air flow over the wing was pulled inboard local to the primary feathers (see Figure 43) over the top surface of the wing. While this flow deviation was observed during visualization testing, the severity of the deviation shown by the pressure rake testing results is nonetheless surprising. It is likely that the feather positioning and increased lift generated at $10^{\circ}$ angle of attack may have exacerbated the effect. Additional studies will need to be conducted to investigate the vortex positioning at a nominal angle of attack of $0^{\circ}$.

\subsection{Total Pressure Probe Results}

While the pressure rake results presented above indicate the presence of vortex structures downstream of the wing, it was determined that additional testing would be carried out to using an alternative approach in an effort to improve the quality of results. To this end, a total pressure probe was used to sample two planes aft of the wing, one at $0.2 \mathrm{~m}$ downstream and one at $0.1 \mathrm{~m}$ downstream. The wing was again placed at an angle of attack of $10^{\circ}$ with a flow velocity of $5 \mathrm{~m} / \mathrm{s}$. Figures 46 and 47 detail the results of the initial total pressure probe test at a distance of $0.2 \mathrm{~m}$ downstream of the model. 

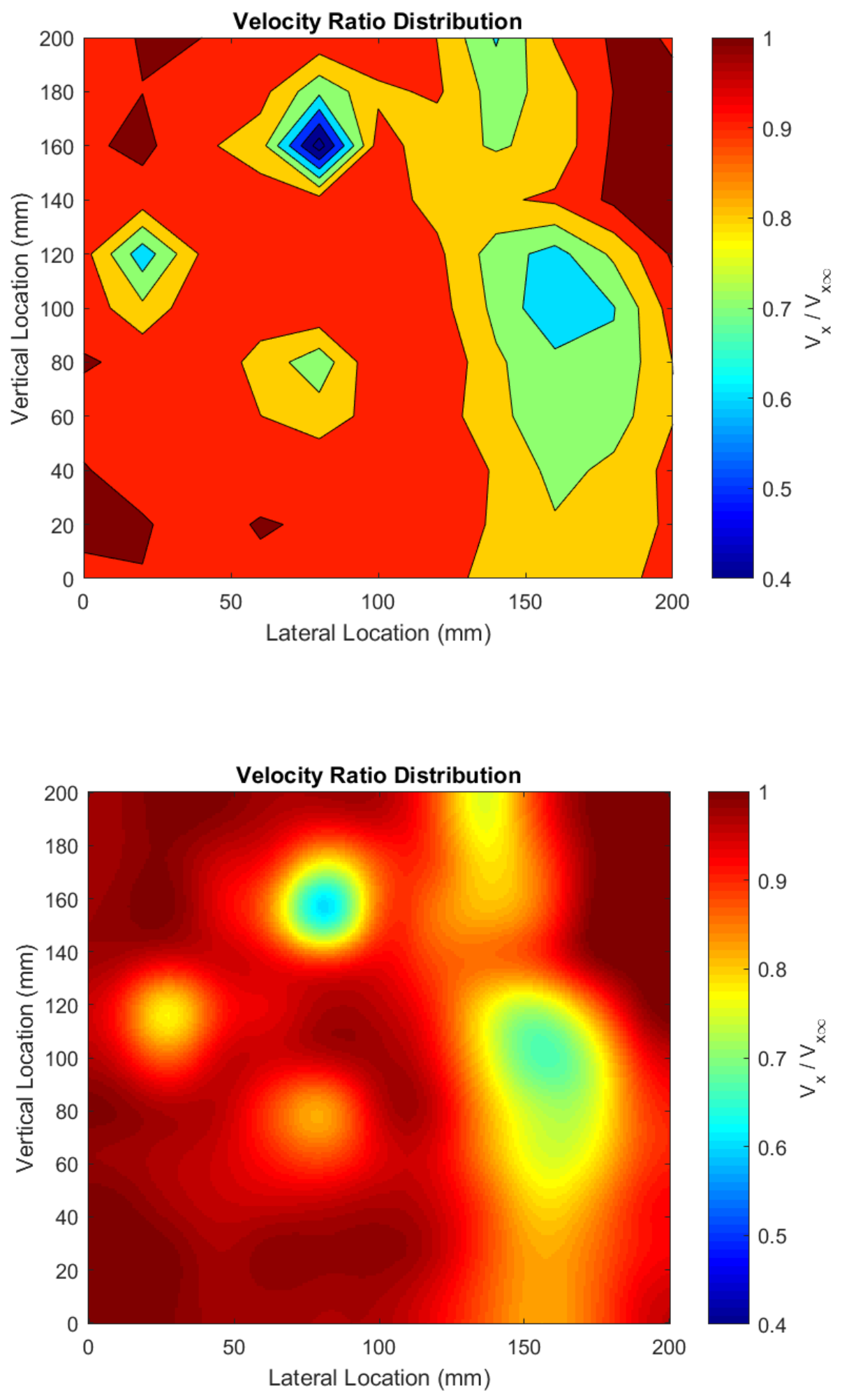

Figure 46. Plot of the velocity ratio $\mathrm{V}_{\mathrm{x}} / \mathrm{V}_{\mathrm{x} \infty}$ generated using a total pressure probe at a distance of $0.2 \mathrm{~m}$ downstream of the model and a flow velocity of $5 \mathrm{~m} / \mathrm{s}$. 

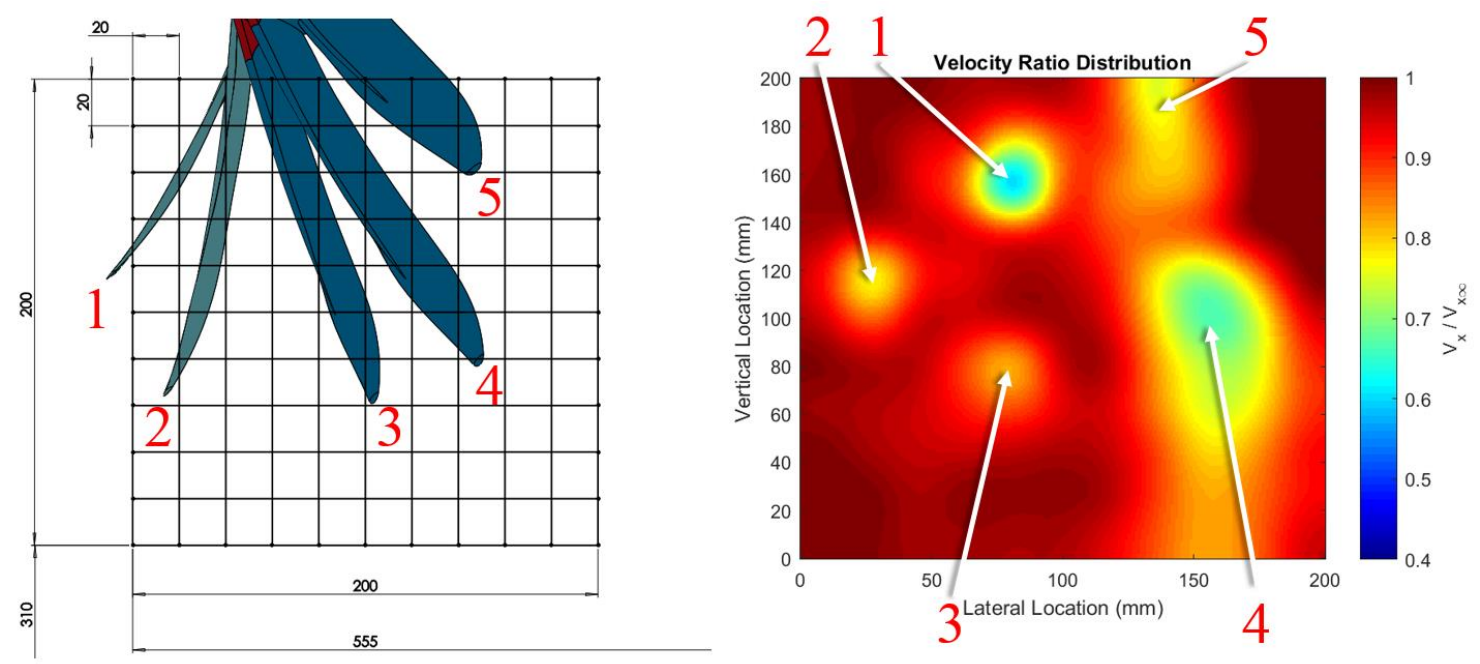

Figure 47. Comparison of the $\mathrm{CAD}$ geometry to data generated during total pressure testing with the sampling plane located $0.2 \mathrm{~m}$ downstream of the model. Each vortex structure and its generating feather are matched by number. All dimensions are in $\mathrm{mm}$.

The increase in quality of the results compared to those produced by the pressure rake is immediately apparent. Additionally, the number of vortices and their locations are clearly consistent between the results produced by the total pressure probe and the pressure rake. Of particular note is the increased resolution of vortices 4 and 5 , as well as the surrounding low pressure areas. The indistinct vortex structures observed during pressure rake testing are here more resolved and noticeable. Vortices 1 and 2 continue to be pulled upward and two the right, mimicking earlier results. The relatively strength of each vortex generally correlates well between the two tests, though the minimum velocity ratio suggested by pressure rake testing is somewhat lower. This is likely to be a result of the coarser sampling resolution of the total pressure probe test, which may have resulted in a situation where no sampling grid locations were located at the core of vortex 1 .

A second plane at a distance of $0.1 \mathrm{~m}$ downstream of the model was then investigated using the total pressure probe using identical test conditions (flow velocity of $5 \mathrm{~m} / \mathrm{s}$, wing angle of attack 
$10^{\circ}$ ). This position was chosen in an effort to characterize the evolution of the vortex structures over time. The number of samples and the sampling grid positioning were refined based on results obtained during the first total pressure probe test. The results of the second test are presented in Figures 48 and 49. 

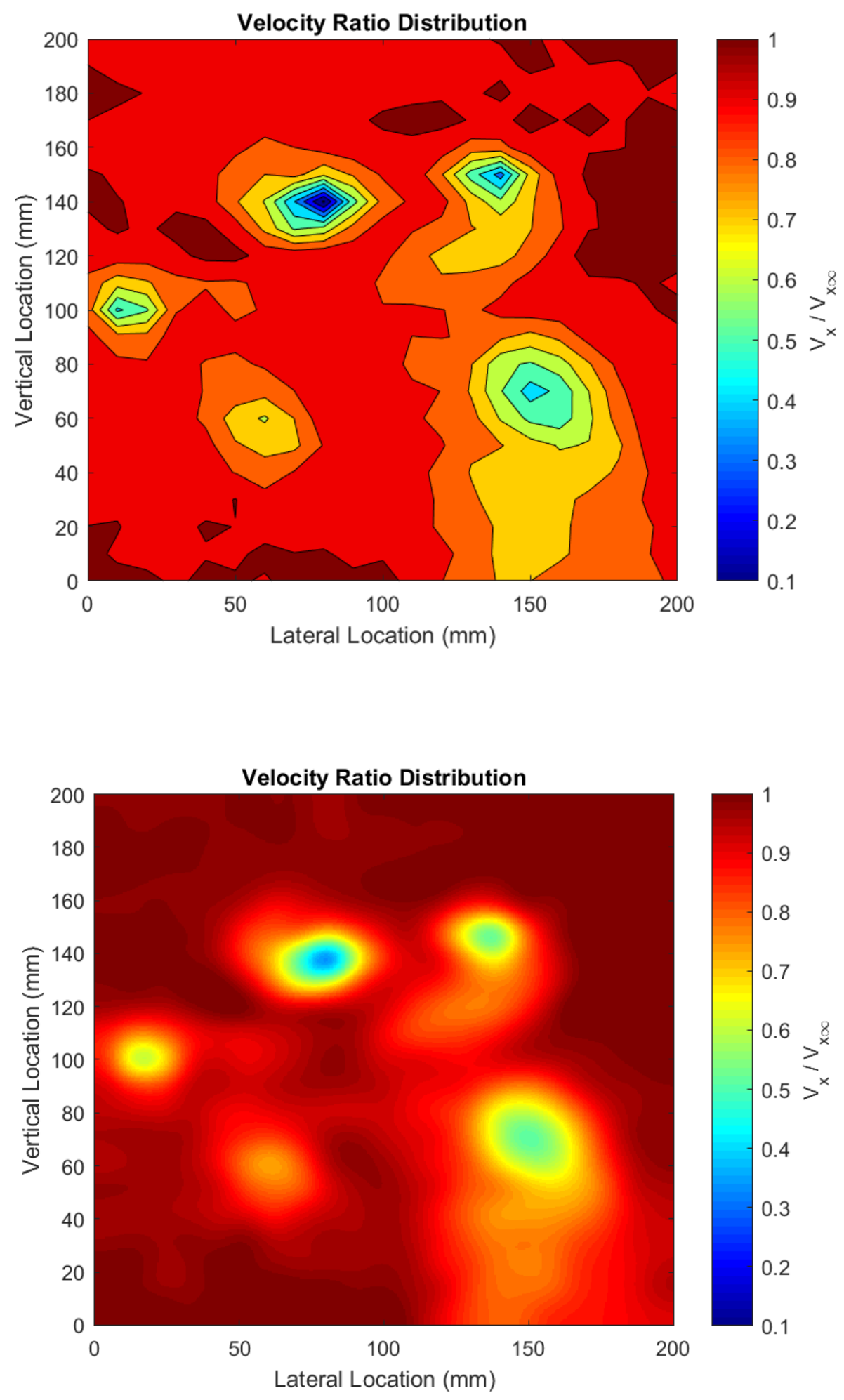

Figure 48. Plot of the velocity ratio $\mathrm{V}_{\mathrm{x}} / \mathrm{V}_{\mathrm{x} \infty}$ generated using a total pressure probe at a distance of $0.1 \mathrm{~m}$ downstream of the model and a flow velocity of $5 \mathrm{~m} / \mathrm{s}$. 

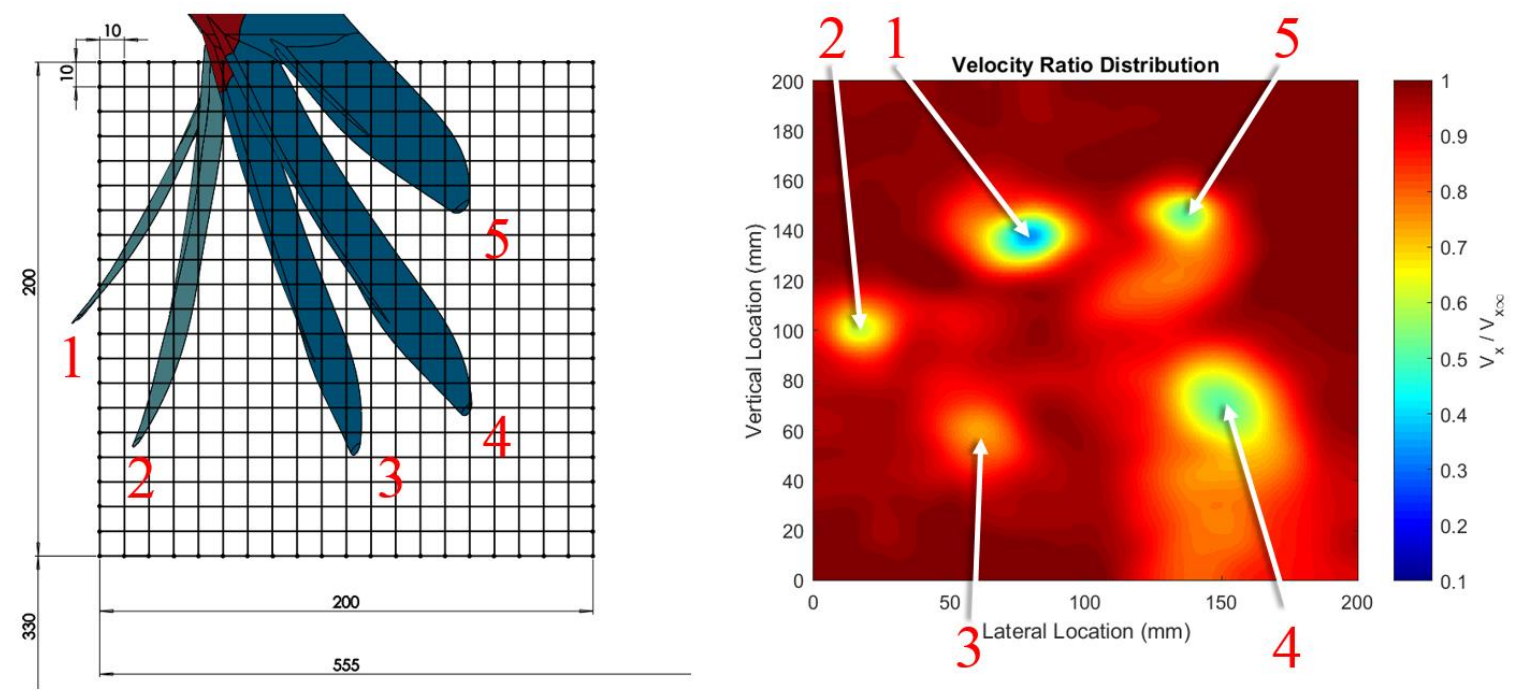

Figure 49. Comparison of the $\mathrm{CAD}$ geometry to data generated during total pressure testing with the sampling plane located $0.1 \mathrm{~m}$ downstream of the model. Each vortex structure and its generating feather are matched by number. All dimensions are in $\mathrm{mm}$.

This round of total pressure probe testing resulted in strong correlation with previous results in terms of vortex positioning and the increase in resolution is again apparent. Of note is the fact that the velocity ratio at the core of each vortex is substantially lower than that observed when using the total pressure probe at a distance of $0.2 \mathrm{~m}$ by a velocity ratio factor of approximately 0.3 . This is likely due to a combination of two factors. Because the sampling plane in this case is closer to the model, the vortices shown in Figures 48 and 49 have had less time to propagate and mix with the surrounding free-stream flow. As such, the flow velocity in the rotational direction at the core of each vortex is likely closer to be much lower than the velocity of the vortex core observed at more distant planes. Additionally, the total pressure probe used in this experiment is only able to resolve flow velocity along the axis of the wind tunnel. Because the free-stream velocity has been transformed into a strong rotational velocity at each vortex structure, the flow velocity apparent to the total pressure probe is therefore much lower than the 
actual total flow velocity. These effects should be less pronounced further downstream as each vortex continues to propagate and mix with the free-stream flow.

\subsection{Experimental Uncertainty}

\subsubsection{Total Pressure Rake}

As noted earlier, a blocked pressure port and artificially low pressure values reported by each end of the rake presented significant difficulties during data collection and post-processing, leading to some concern regarding the quality of the resulting data. While the source of error is reasonably clear when dealing with a blocked pressure port, the cause of unusually low pressure readings at the ends of the rake is somewhat less obvious. While troubleshooting is ongoing, it is probable that the structure of the rake itself interfered with the natural propagation of the airflow and vortex structures during testing. Additionally, the low free-stream velocity chosen for this study may have been a poor match for the resolution of the Scanivalve pressure measurement system. The difficulties encountered during pressure rake testing ultimately led to the decision to conduct future pressure mapping studies using an alternative approach.

In an effort to quantify the amount of potential error during the sampling process, the standard deviation of the 100 data points sampled by each port at each sampling location was calculated. The resulting contour plot of the standard deviation throughout the flow field for the test utilizing the total pressure rake is presented in Figure 50. 


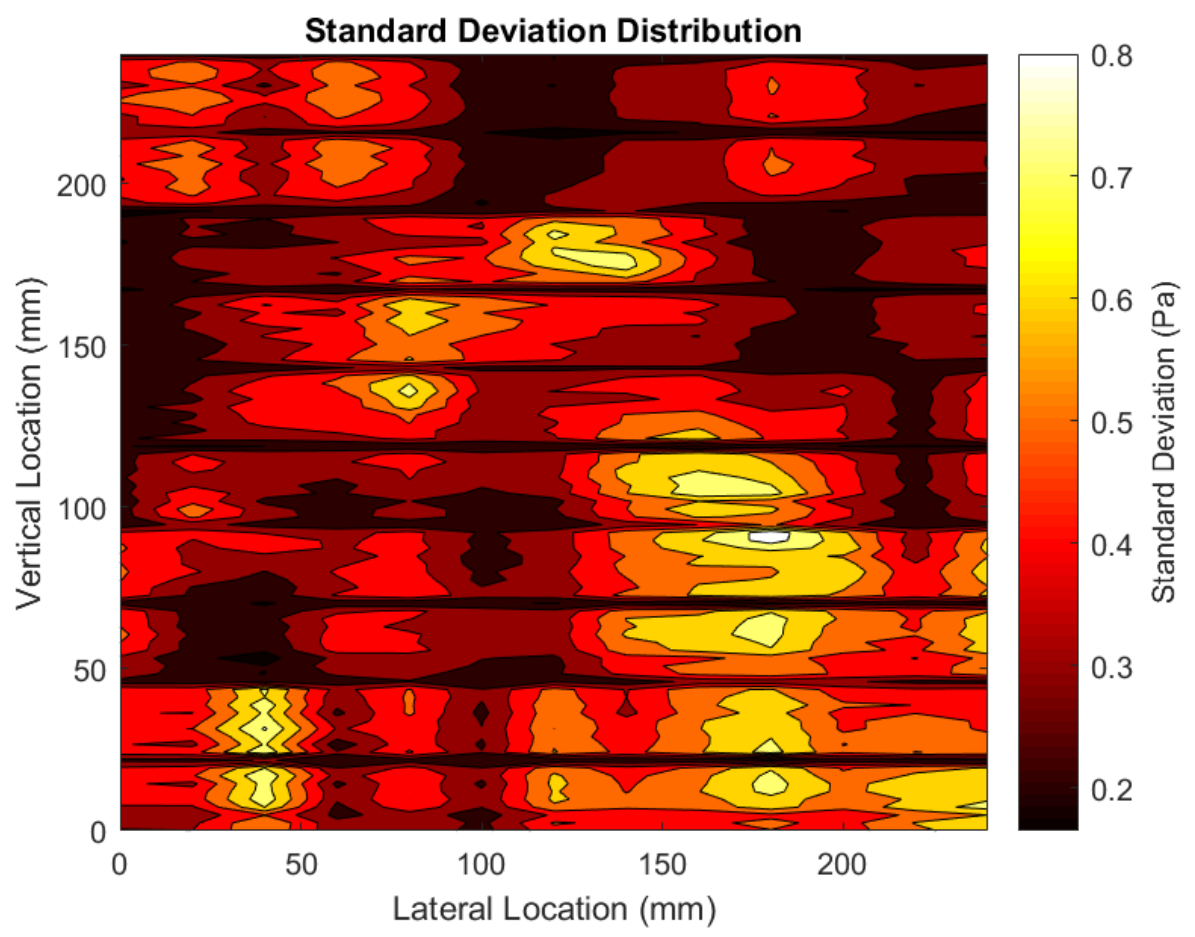

Figure 50. Contour plot of the standard deviation of each data sample collected by the total pressure rake.

The maximum pressure reading among the collected points was $15.605 \mathrm{~Pa}$, whereas the minimum reading was 0.895 . This led to a sampling range of $14.710 \mathrm{~Pa}$. It can therefore be concluded that the maximum standard deviation $(0.8 \mathrm{~Pa})$ among the samples collected throughout the flow field represented approximately $5.4 \%$ of the sampling range, a number low enough to provide confidence in the consistency of each port's sampling ability. However, due to a number of other issues encountered during usage of the pressure rake, the results are presented primarily for informational purposes and to provide context for later testing.

\subsubsection{Total Pressure Probe}

While results obtained while using the total pressure probe resulted in a marked increase in data quality when compared to the total pressure rake results, it should be noted that this approach 
is ideal for linear flows where the axis of the probe is aligned with the direction of the flow. Matters are somewhat more complicated when investigating rotational flows (such as the vortex flows investigated here) as the probe is only able to resolve flow velocity along its axis when the total pressure port alone is used. Work to establish a calibration matrix allowing for accurate reporting by all five ports is ongoing and will allow future studies to resolve both the speed and direction of flows, providing a more accurate and complete view of the vortex geometry produced by the wing's primary feathers.

The amount of potential error was here quantified in a manner similar to that described in section 9.5.1. The resulting contour plot for the test plane at $0.1 \mathrm{~m}$ downstream is presented below.

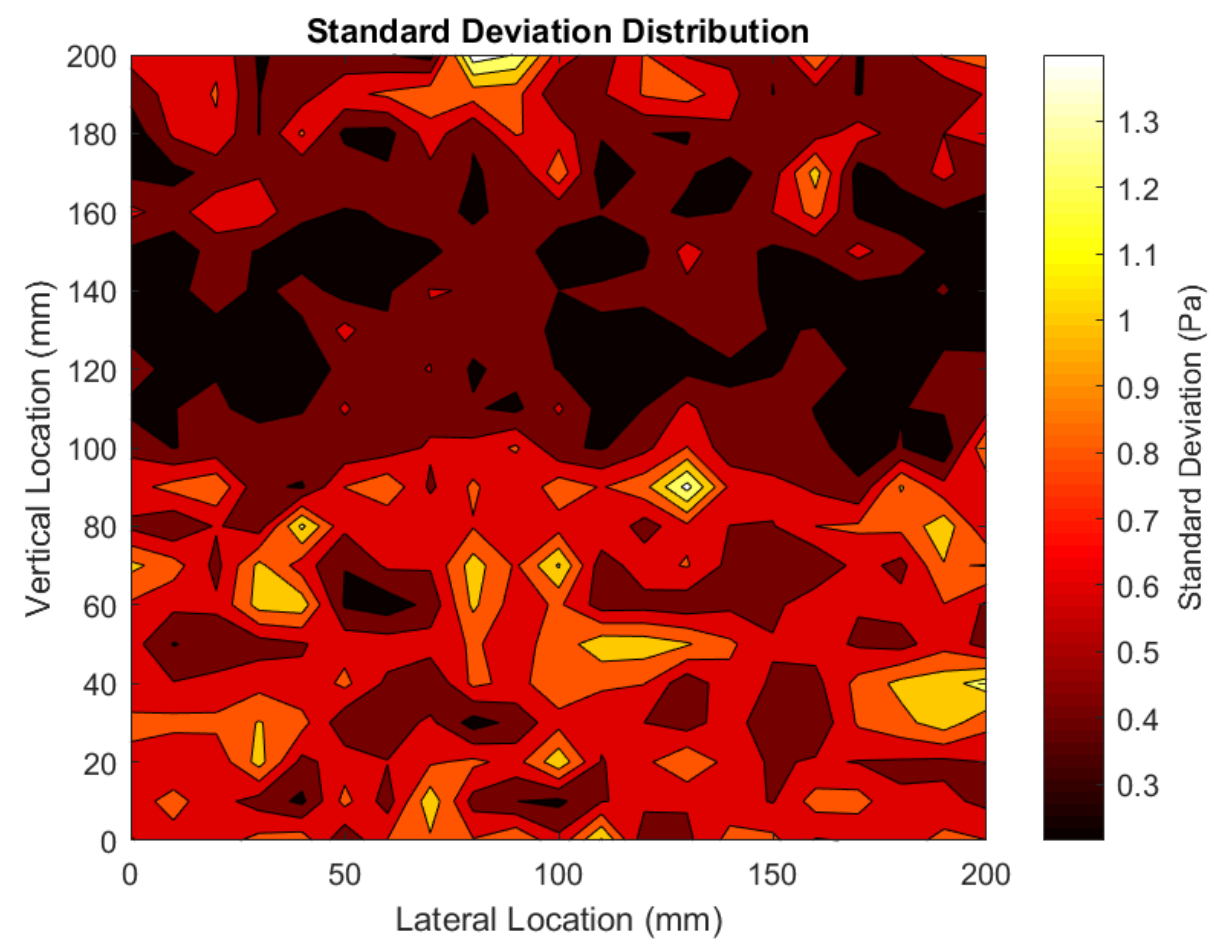

Figure 51. Contour plot of the standard deviation of each data sample collected by the total pressure probe at a distance of $0.1 \mathrm{~m}$ downstream. 
In this case, the maximum pressure reading among the collected points was $16.833 \mathrm{~Pa}$, whereas the minimum reading was 0.095 , leading to a sampling range of $16.738 \mathrm{~Pa}$. The maximum standard deviation (1.38) here represents approximately $8.2 \%$ of the sampling range, leading to a reasonably high degree of confidence regarding the consistency of samples collected by the total pressure probe.

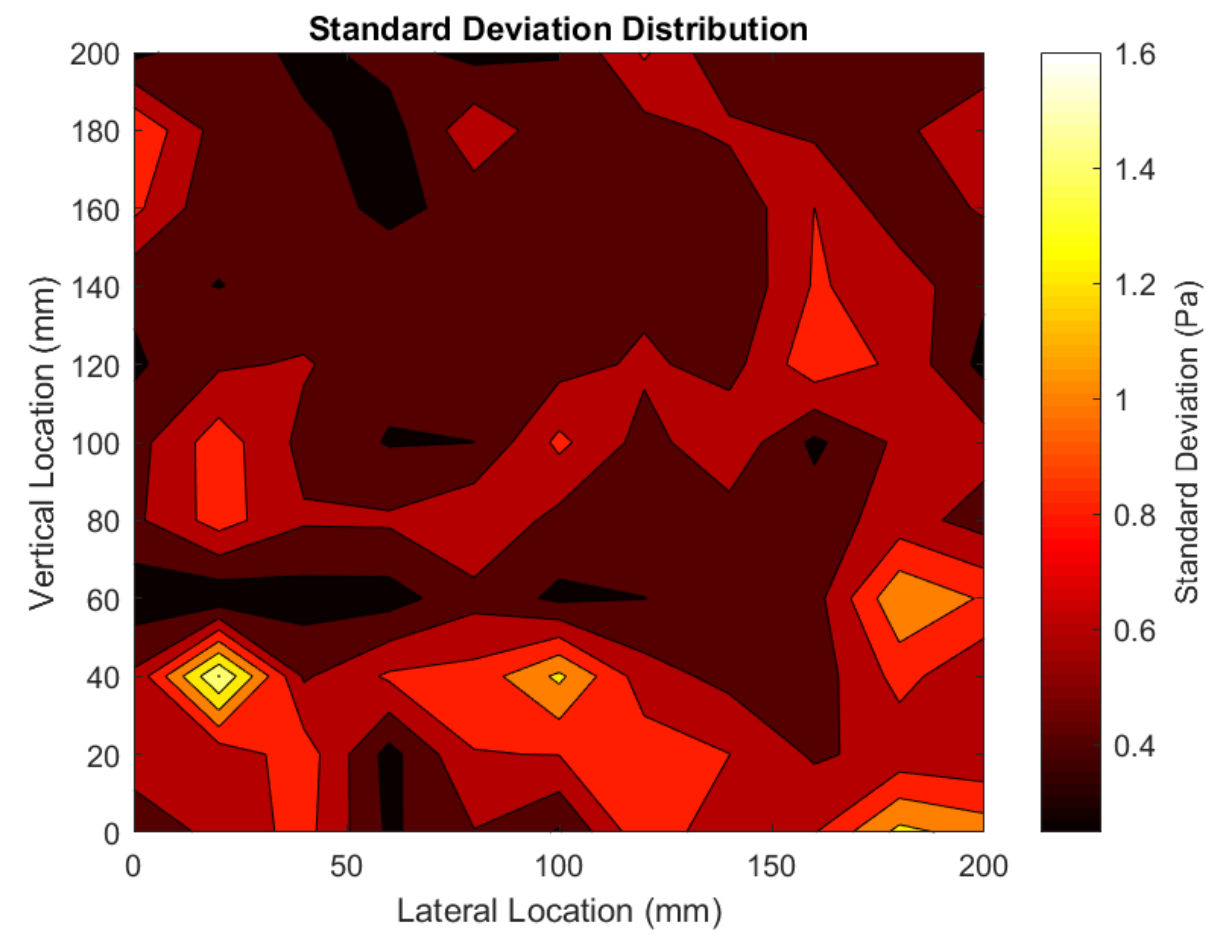

Figure 52. Contour plot of the standard deviation of each data sample collected by the total pressure probe at a distance of $0.2 \mathrm{~m}$ downstream.

The maximum pressure reading among the collected points at $0.2 \mathrm{~m}$ downstream was 15.665 $\mathrm{Pa}$, whereas the minimum reading was 1.774 . This led to a sampling range of $13.891 \mathrm{~Pa}$, with the maximum standard deviation (1.6) representing approximately $11.5 \%$ of the sampling range. The maximum standard deviation here is somewhat higher than that calculated for the prior total pressure probe test, though this is likely to be primarily due to an outlier data point located in 
the bottom left corner of the flow field. Filtering this outlier out results in a similar degree of confidence between the two tests.

\subsubsection{Vibration}

Prior to beginning wind tunnel testing, a study was conducted to quantify any deflections or vibrations of the model under load using both image comparison and video footage. Photos and videos were obtained using a Nikon D3200 HD-SLR camera mounted on a tripod approximately $1.45 \mathrm{~m}$ downstream of the model. Both the $0^{\circ}$ and $10^{\circ}$ angle of attack configurations were investigated. However, there was virtually no discernable deflection for either case. A small amount of vibration at the tip of the fourth feather was noted in both cases, though this was found to be small in amplitude (on the order of $1 \mathrm{~mm}$ laterally left and right) and was not periodic. It was determined that this vibration was likely caused by unsteady flow generated by the upwind feathers. This slight vibration, while small, could help to explain the more indistinct shape of the vortex generated by the fourth feather as observed during testing.

Both the pressure rake and total pressure probe were securely mounted to the traverse and checked for vibration at a flow velocity of $5 \mathrm{~m} / \mathrm{s}$. No discernable vibration of either measurement device was detected. 


\section{CONCLUSIONS AND RECOMMENDATIONS}

\subsection{Conclusions}

A novel process to develop the most biologically accurate model of a slotted primary feather geometry constructed to date was investigated and defined using a combination of 3D scanning, 3D printing and CAD technologies. To test the derived process, an aerodynamic test model was successfully created and tested in the Cal Poly low speed wind tunnel at a flow velocity of $5 \mathrm{~m} / \mathrm{s}$ and wing angle of attack of $10^{\circ}$. Flow characterization was carried out using a combination of smoke and laser visualization coupled with total pressure rake and total pressure probe testing.

Before beginning testing, characterization of several airfoil sections generated using 3D scan data of a Brown Pelican wing was carried out. The resulting measurements were compared to an existing study detailing the morphology of the Australian Pelican (Pelecanus conspicillatus). While several discrepancies did exist, the resulting measurements correlated well with the results of the prior study and with published literature detailing the skeletal and muscular layout of the wings of Brown Pelicans, providing a high degree of confidence regarding the representativeness and veracity of the preserved Brown Pelican wing. However, the specimens compared here should not be assumed to be wholly representative of both pelican species, but rather two distinct individuals. Additional studies will need to be conducted to obtain more average measurements and airfoil sections for comparison.

Flow visualization at a distance of $0.2 \mathrm{~m}$ downstream of the model revealed that vortices were created by multiple feather tips as expected. In addition, a large-scale pressure gradient caused by the generation of high pressure under the wing and lower pressure over the wing caused the airflow above the primary feathers to curve inboard towards the wing root, while the flow below the feathers was simultaneously deflected outwards. This visualization testing provided invaluable data and provided crucial information that was used to clarify results suggested by later pressure tests. 
Aerodynamic testing using a total pressure rake at a distance of $0.2 \mathrm{~m}$ downstream confirmed the presence of multiple clearly defined vortex structures in the wake of the model. These were visualized by plotting the velocity ratio $\mathrm{V}_{\mathrm{x}} / \mathrm{V}_{\mathrm{x} \infty}$, or the ratio of the local velocity along the axis of the wind tunnel at the probe tip to the free-stream velocity. Of particular note was the position of the vortices created by the first and second feather. Both vortices were deflected upwards and to the right, correlating well with the flow deviation around the primary feathers noted during smoke visualization experiments. However, some testing difficulties lead to additional pressure testing being carried out using a total pressure probe.

Subsequent testing using a total pressure probe at a similar sampling plane and flow velocity provided a marked increase in resolution and data quality. A plot of the velocity ratio $\mathrm{V}_{\mathrm{x}} / \mathrm{V}_{\mathrm{x} \infty}$ was again created and compared to previous pressure rake results. While the positioning of each vortex and the surrounding low pressure areas correlated very well with results produced during prior testing, the relative strength of each vortex structure was slightly higher than predicted by the pressure rake by a velocity ratio factor of approximately 0.10 . This result is explained by the relative coarseness of the total pressure probe test when compared to the total pressure rake test and it is likely that the core of vortex 1 was not adequately captured by the probe.

A final total pressure probe test at a distance of $0.1 \mathrm{~m}$ downstream yielded results consistent with the previous two tests. However, the velocity ratio distribution of each vortex core was found to be significantly lower than that recorded at a distance of $0.2 \mathrm{~m}$ by a velocity ratio of approximately 0.3 . This is likely due to the fact that the vortices have had less time to mix with the free stream flow, leading to a vortex core velocity that is much closer to zero in the rotational direction. As the vortex structures propagate downstream, the rotational velocity will be slowly converted to free-stream velocity, which subsequently results in a higher velocity ratio. In addition, the total pressure probe used is only capable of resolving flow velocity along the axis of the wind 
tunnel. Because the flow velocity in these newly-formed vortices is primarily rotational instead of free-stream, the velocity resolved by the total pressure probe will appear to be lower.

An additional study was carried out to investigate the flex of a Brown Pelican primary feather at various flow velocities, angles of attack and sweep angles. The results of this study may be found in Appendix A.

\subsection{Simplifications and Recommendations for Future Work}

This study focuses on characterizing the aerodynamic effects of a single, static geometry of slotted primary wing tip feathers of the Brown Pelican. In reality, avian feathers are dynamic structures and can not only be articulated by the pelican's skeletomuscular system, but may also twist and flex under aerodynamic loading. A prime example of this can be found in the primary feather deflection experiment described in Appendix A. Future studies will investigate different Brown Pelican primary feather configurations and flow velocities in an effort to characterize the effect of feather position on vortex generation and potential drag reduction in various flight configurations. A properly calibrated five-hole probe could provide valuable data regarding the vorticity downstream of the model, further clarifying the strength of each vortex.

It should be noted that all primary feathers on the test model were created using the single primary feather as reference. In actuality, each primary feather has a slightly different geometry (as can be seen in Figure 6). Additionally, the wing and feathers were modeled as perfectly smooth objects, which is certainly not the case for their biological counterparts. Future studies will investigate various surface textures mimicking that of an avian feather, with the textures either created during the $3 \mathrm{D}$ printing process or as a secondary operation.

Finally, 3D scans of the wing and primary feather of a California Condor obtained during this project could ultimately lead to a similar series of studies investigating the aerodynamic characteristics of the condor's wing tip feathers. The model creation process, analytical tools and 
wind tunnel testing results outlined here provide a strong foundation for the continued elucidation of our understanding of the aerodynamics of avian flight and the potential aerodynamic benefits offered by the slotted primary feather geometry. 


\section{REFERENCES}

1. Guerro, J. E., Maestro, D., Bottaro, A. "Biomimetic Spiroid Winglets for Lift and Drag Control.” Comptes Rendus Mechanique, Vol. 340, No. 1-2, 2011, pp. 67-80.

2. Chambers, Joseph R. Concept to Reality: Contributions of the NASA Langley Research Center to U.S. Civil Aircraft of the 1990's. NASA History Series, NASA SP-2003-4529, 2003.

3. Terres, John K. The Audubon Society Encyclopedia of North American Birds. Alfred A. Knopf, Inc., 1980.

4. Shields, Mark A. "Brown pelican: pelecanus occidentalis." Birds of North America, No. 609, 2002, pp. 1-35.

5. Sprunt, Alexander and E. Burnham Chamberlain, South Carolina Bird Life. University of South Carolina Press, 1970.

6. Schulenburg, Frank. "Juvenile pelecanus occidentalis in flight.” Wikipedia, 2016. Accessed 5 May 2017.

7. Palmer, Ralph S. Handbook of North American Birds. Vol. 1. Yale University Press, 1962.

8. Anderson, John D. Fundamentals of Aerodynamics. McGraw-Hill, 2001.

9. Lanchester, F. W. Aerodynamics, Constituting the First Volume of a Complete Work on Aerial Flight, Aerodonetics, Constituting the Second Volume. Archibald Consff \& Co. Ltd., 1907.

10. Traill, Richard M. A Preliminary Investigation of Fixed Wing Flight of the Australian Pelican with Reference to the Ground Effect. Master's thesis, University of New South Wales, 2005.

11. Abbott, Ira H. and Albert E. von Doenhoff. Theory of Wing Sections. Dover Publications, 1959.

12. Coiro, D. P., Nicolosi, F., Scherillo, F., Maisto, U. "Single Versus Multiple Winglets: Numerical and Experimental Investigation." 26th International Congress of the Aeronautical Sciences, 2008.

13. Catalano, F. M., Ceron-Munoz, H.D. "Experimental Analysis of Aerodynamic Characteristics of Adaptative Multi-Winglets." 24th International Congress of the Aeronautical Sciences, 2004.

14. Cosin, R., Catalano, F.M., Correa, L. G. N., Entz, R. M. U. "Aerodynamic Analysis of MultiWinglets for Low Speed Aircraft." 27th International Congress of the Aeronautical Sciences, 2010 .

15. Ursache, N.M., Melin, T., Isikveren, A. T., Friswell, M. I. "Morphing Winglets for Aircraft Multi-Phase Improvement." $7^{\text {th }}$ AIAA Aviation Technology, Integration and Operations 
Conference, AIAA No. 2007-7813, 2007.

16. Shelton, A., Tomar, A., Prasad, J. V. R., Smith, M. J., Komerath, N. "Active Multiple Winglets for Improved UAV Performance." 22nd Applied Aerodynamics Conference and Exhibit, 2004.

17. Schnell, G.D., Hellack, J. D. "Bird Flight Speeds in Nature: Optimized or a Compromise?" The American Naturalist, Vol. 113, No. 1, 1979, pp. 53-66.

18. Hedenstrom, A., Alerstam, T. “Optimal Flight Speeds of Birds." Philosophical Transactions: Biological Sciences, Vol. 348, No. 1326, 1995, pp. 471-487.

19. Spedding, G. R. "The Wake of a Kestrel (falco tinnunculus) in Gliding Flight." Journal of Experimental Biology, Vol. 127, 1987, pp. 45-57.

20. Spedding, G. R. "The Wake of a Jackdaw (corvus monedula) in Slow Flight." Journal of Experimental Biology, Vol. 125, 1986, pp. 287-307.

21. Chamberlain, Frank W. Atlas of Avian Anatomy. Michigan State College, 1934.

22. Simons, E. L. R., Hieronymus, T. L., O'Connor, P. M. "Cross Sectional Geometry of the Forelimb Skeleton and Flight Mode in Pelecaniform Birds.” Journal of Morphology, Vol. 272, 2011, pp. 958-971.

23. Gill, F. B. Ornithology. W. H. Freeman and Company, 2007.

24. "Brown Pelican primary wing feathers." U.S. Fish and Wildlife Service Forensics Laboratory, 2015. Accessed 19 May 2017.

25. Tucker, V. A. "Drag Reduction by Wing Tip Slots in a Gliding Harris' Hawk, parabuteo unicinctus." The Journal of Experimental Biology, Vol. 198, 1995, pp. 775-781.

26. Rosen, M., Hedenstrom, A. "Gliding Flight in a Jackdaw: A Wind Tunnel Study." The Journal of Experimental Biology, Vol. 204, 2001, pp. 1153-1166.

27. "Innovations in Bonding to Low Surface Energy Materials." 3M, 2015. http://multimedia.3m.com/mws/media/755526O/innovations-in-bonding-to-low-surfaceenergy-white-paper.pdf

28. Barlow, Jewel B., William H. Rae and Alan Pope. Low-Speed Wind Tunnel Testing. Wiley, 1999. 


\section{APPENDICES}

\section{A. Feather Deflection Study}

In the case of this study, the aerodynamic test model can essentially be assumed to be a rigid structure. In reality, avian wings and feathers are dynamic structures with a highly variable morphology based on coordinated movement provided by the skeletomuscular system and the twist and flex of individual feathers during flight. To better understand the deflections experienced by the primary feathers of Brown Pelicans in flight and to gather information for possible future projects, a simple aerodynamic study was conducted in the Cal Poly low speed wind tunnel.

A Brown Pelican primary feather (P-9 on the left wing, second feather from the front) provided by the Santa Barbara Museum of Natural History was held by a small clamp stand. This stand was mounted in the wind tunnel such that the feather was centered in the test section laterally and as close to the test section centerline as possible vertically. The quill was wrapped with a rubber band and a strip of $1 \mathrm{~cm}$ thick foam tape to lessen the force exerted by the clamp jaws on the feather and to prevent any potential damage or movement.

The feather was tested in four different configurations: at a nominal $0^{\circ}$ angle of attack (where the vane of the feather was placed as flat as possible), a slightly positive angle of attack, a slightly negative angle of attack and $0^{\circ}$ angle of attack with approximately $12^{\circ}$ of sweep. After focusing the camera at the correct area using a rectangular reference grid, images were taken using two different cameras to obtain both a side view and top view. The results of each test are presented below. Each square of the measurement grid represents $1 \mathrm{~cm} \times 1 \mathrm{~cm}$. 
a)
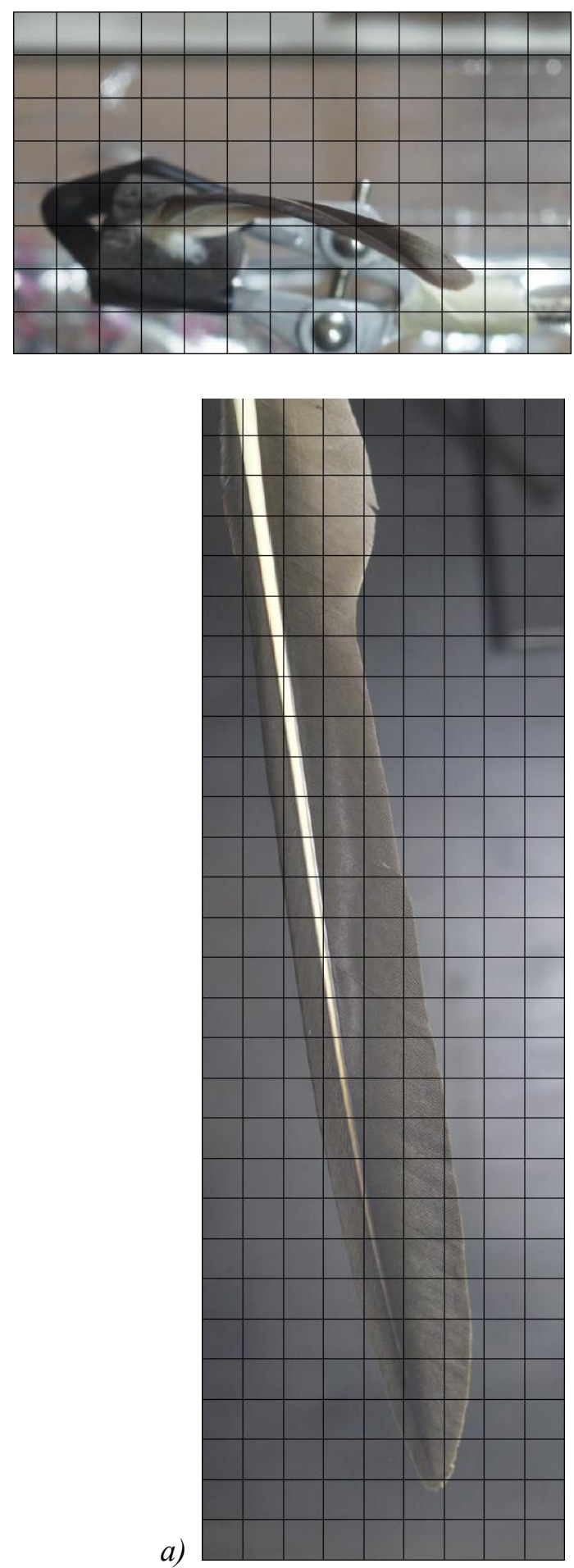

b)
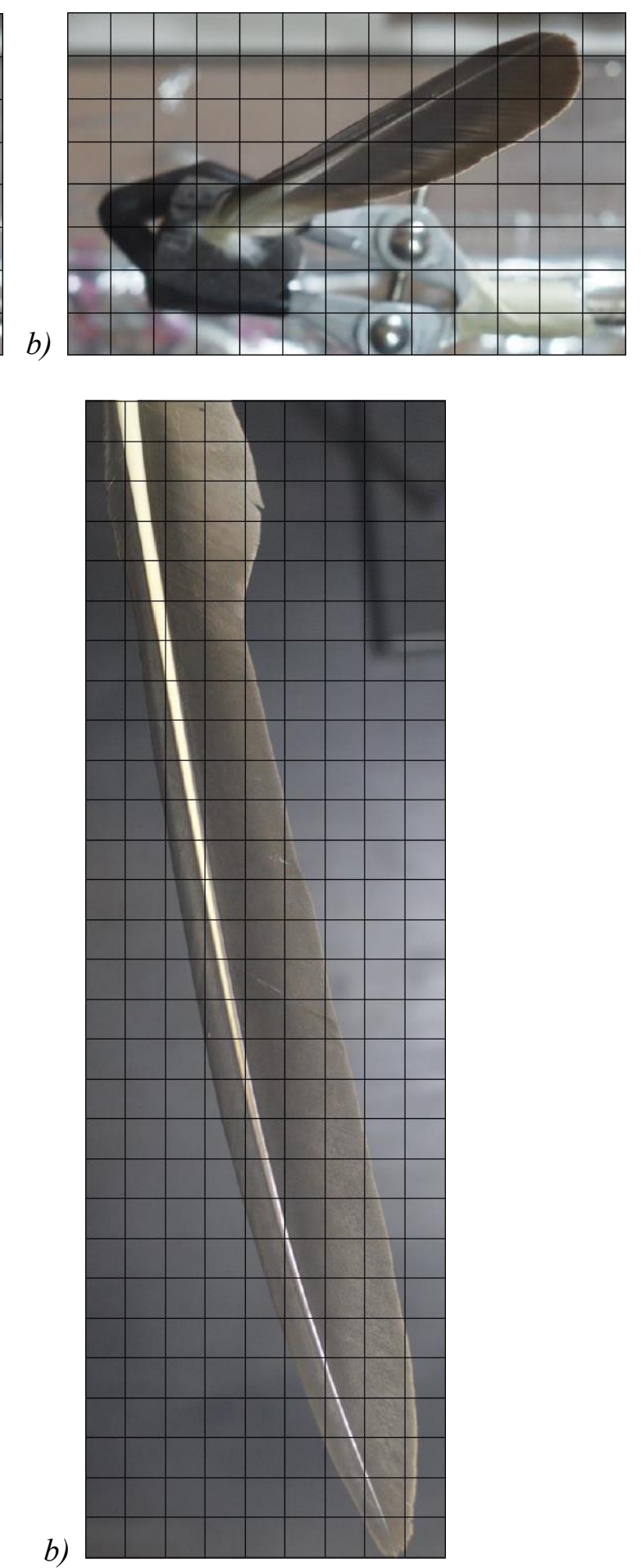

Figure A.1. Side and top views of a Brown Pelican primary feather at $0^{\circ}$ angle of attack: a) flow velocity of $5 \mathrm{~m} / \mathrm{s}$ and b) flow velocity of $10 \mathrm{~m} / \mathrm{s}$. 
Between the $5 \mathrm{~m} / \mathrm{s}$ and $10 \mathrm{~m} / \mathrm{s}$ configuration, the feather tip deflects approximately $6 \mathrm{~cm}$ upwards and $2 \mathrm{~cm}$ aft. Note that at the pelican's cruising speed of $10 \mathrm{~m} / \mathrm{s}$, the feather displays a significant amount of flex, which is likely due to the lift generated. A slight amount of sweep during loading is clearly visible from the top view as well, though this occurs gradually over the span of the feather unlike sweep in a conventional aircraft. 

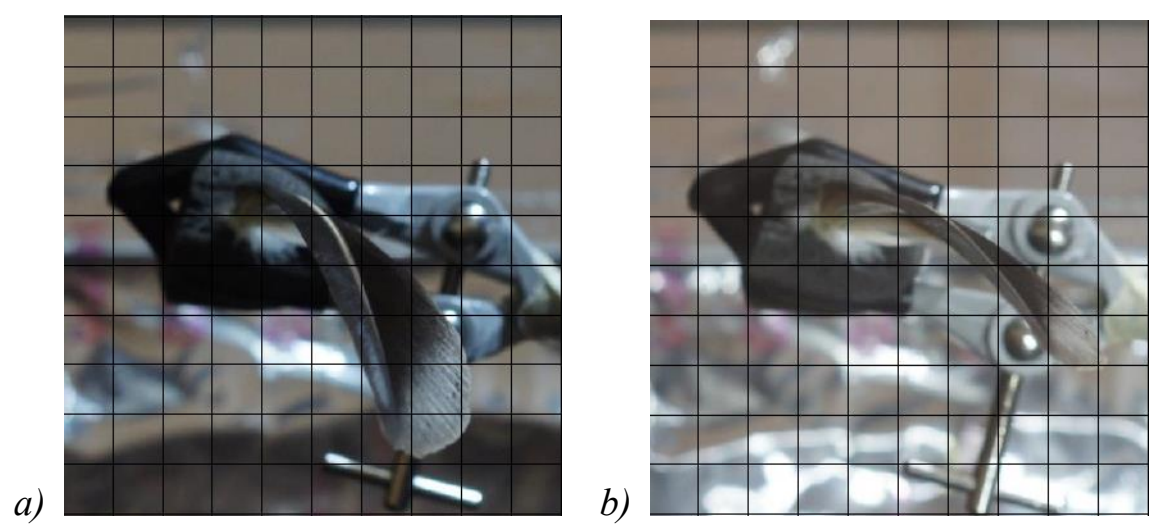

c)
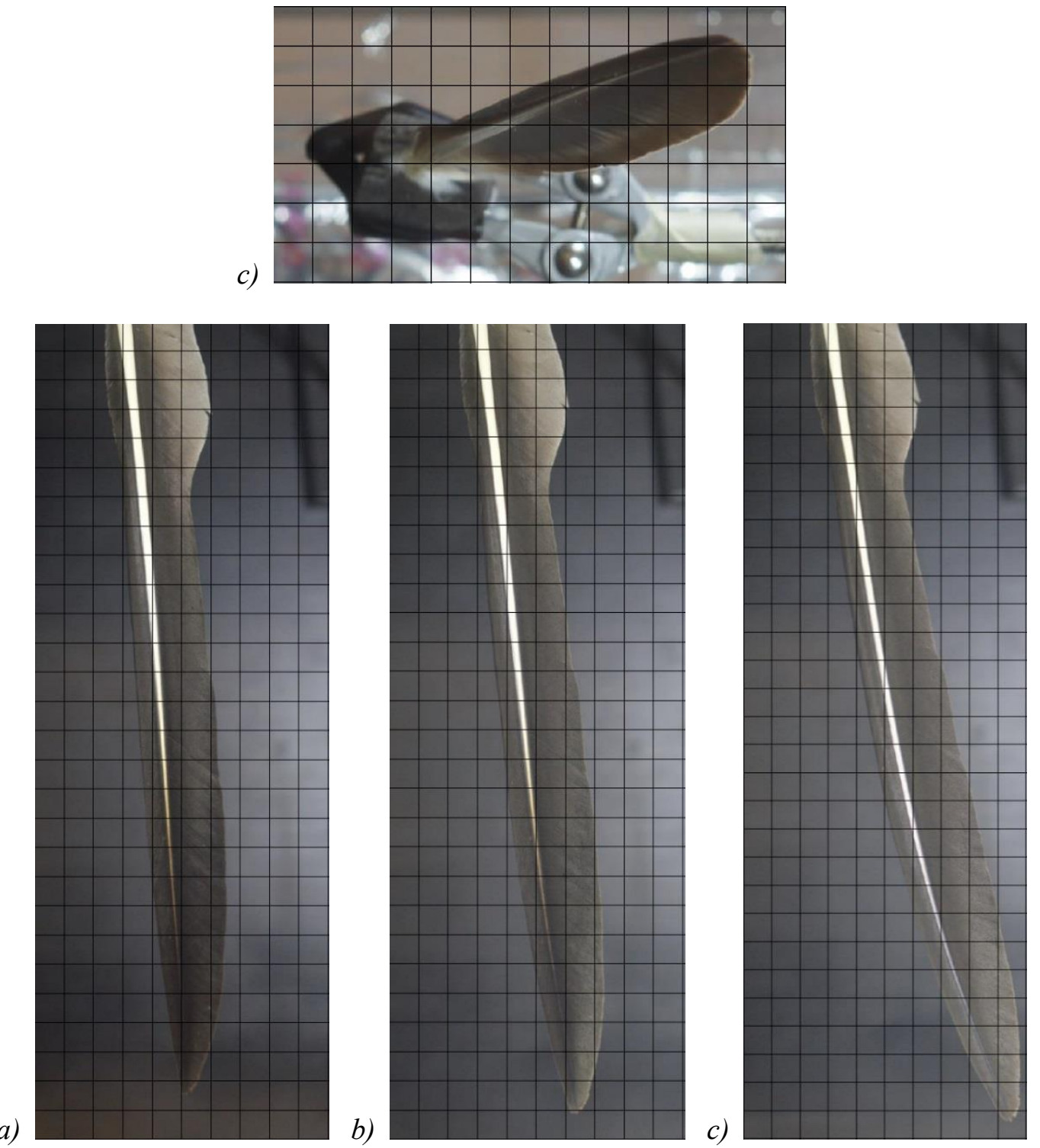

Figure A.2. Side and top views of a Brown Pelican primary feather at a slightly positive angle of attack: a) wind off, b) flow velocity of $5 \mathrm{~m} / \mathrm{s}$ and c) flow velocity of $10 \mathrm{~m} / \mathrm{s}$. 
Between the wind off and $10 \mathrm{~m} / \mathrm{s}$ configuration, the feather tip deflects $8.5 \mathrm{~cm}$ upwards and $3 \mathrm{~cm}$ aft, approximately $45 \%$ more than the $0^{\circ}$ angle of attack case. In addition to the lift generated by the feather, airflow impingement on the underside of the feather likely increases the amount of deflection when compared to the previous case. As before, a slight amount of sweep can be observed from the top view. 
a)
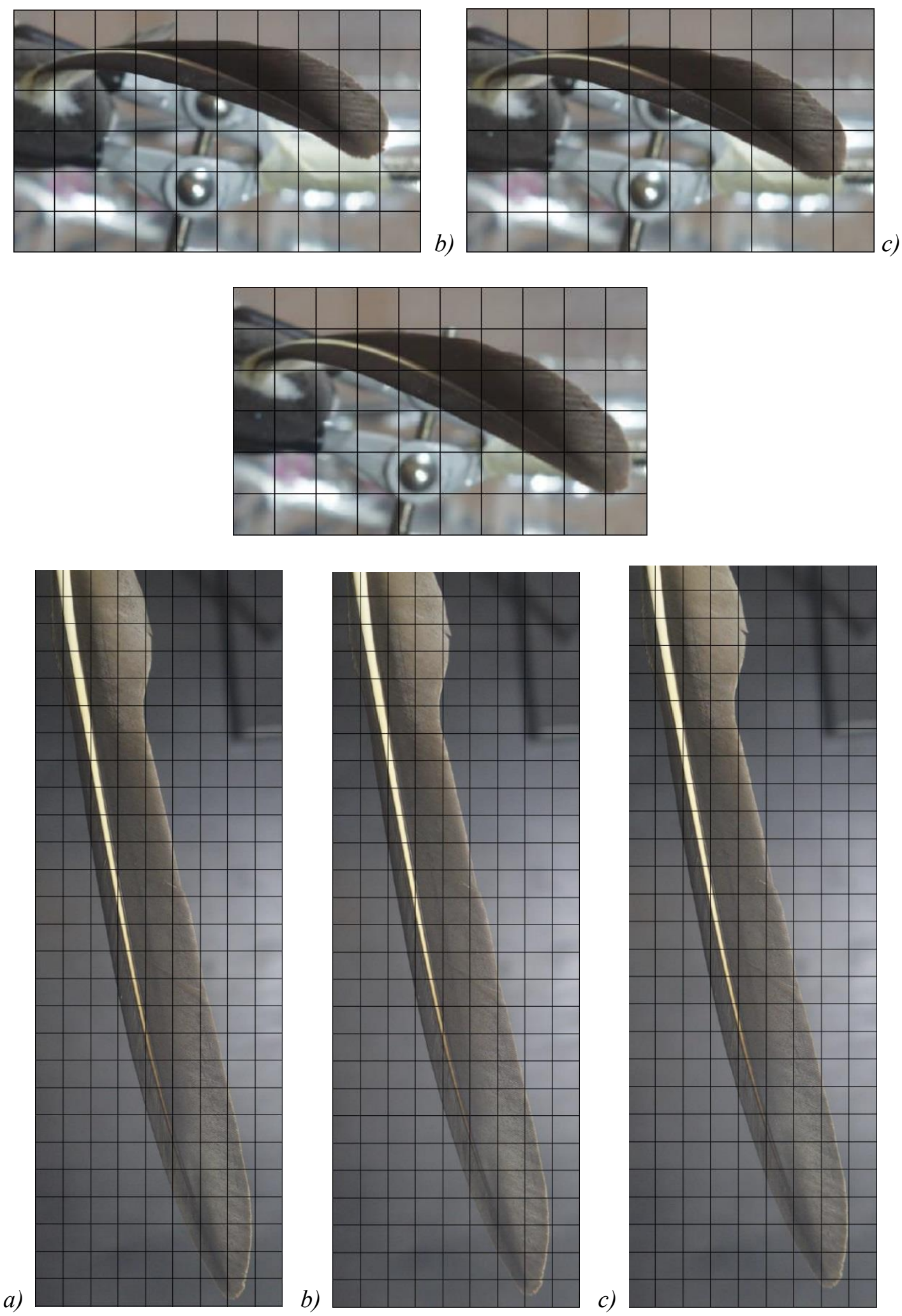

Figure A.3. Side and top views of a Brown Pelican primary feather at a slightly negative angle of attack: a) wind off, b) flow velocity of $5 \mathrm{~m} / \mathrm{s}$ and c) flow velocity of $10 \mathrm{~m} / \mathrm{s}$. 
When placed at a slightly negative angle of attack, the feather tip deflects approximately $1.5 \mathrm{~cm}$ downwards and $0.2 \mathrm{~cm}$ aft. While using a positive angle of attack led to a large amount of vertical flex, a negative angle of attack clearly leads to significantly less flex. These results are to be expected as feathers are rarely loaded in this fashion and are more resistant to flexing in this direction. The deflection in this case is primarily due to the impingement of the flow on the upper surface of the feather. 
a)

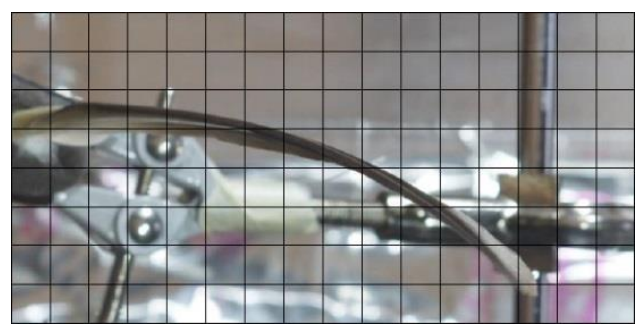

b)

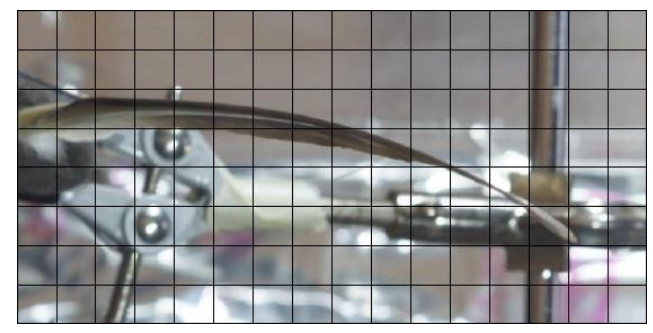

c)
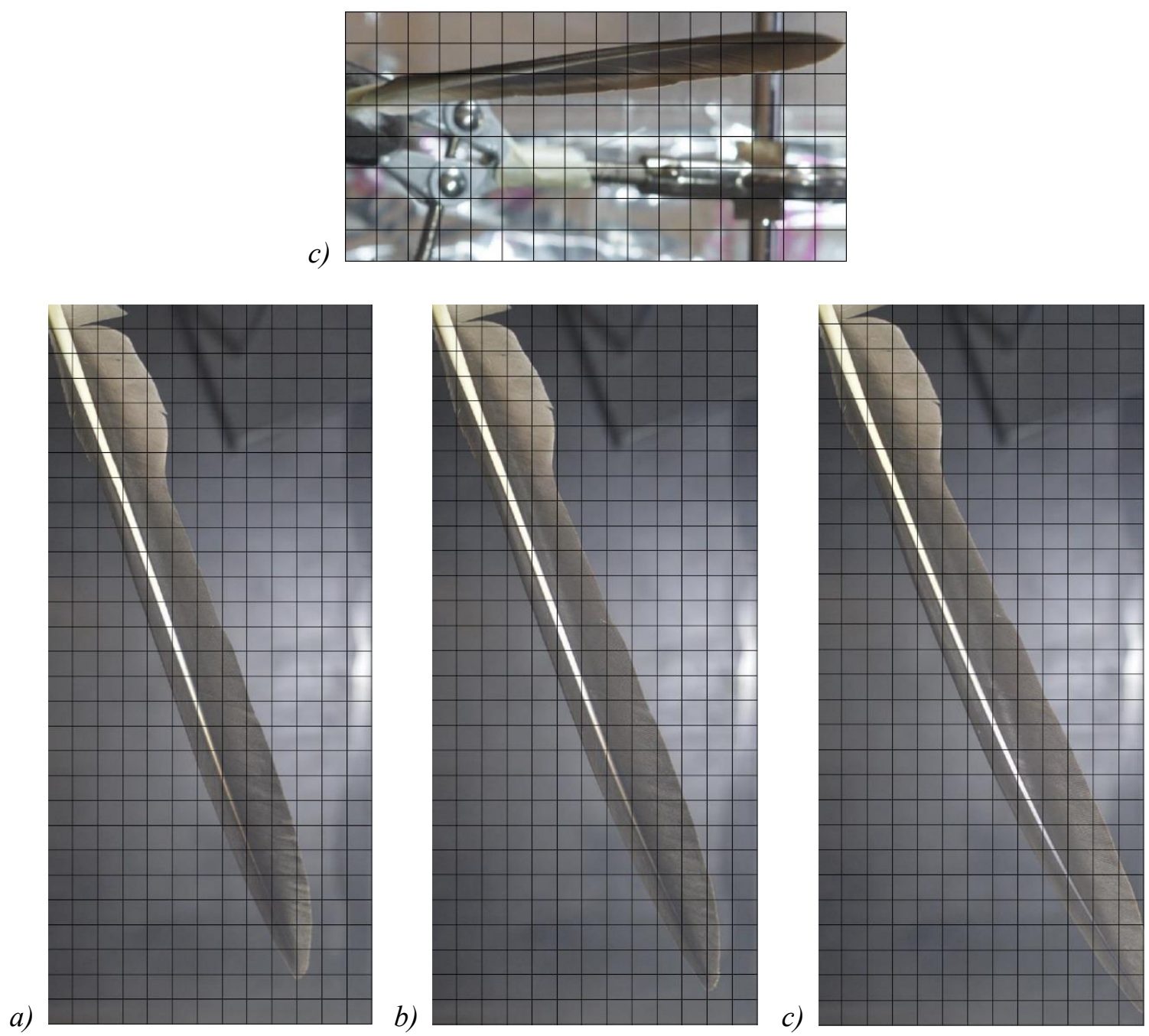

Figure A.4. Side and top views of a Brown Pelican primary feather at $0^{\circ}$ angle of attack and $12^{\circ}$ backwards sweep: a) wind off, b) flow velocity of $5 \mathrm{~m} / \mathrm{s}$ and c) flow velocity of $10 \mathrm{~m} / \mathrm{s}$. 
In this configuration, the feather tip deflects approximately $6.5 \mathrm{~cm}$ upwards and $3 \mathrm{~cm}$ aft. Depending on the gliding configuration of the pelican, it is not at all unusual to observe primary feathers with some degree of rearwards sweep. At a flow velocity of $10 \mathrm{~m} / \mathrm{s}$, the familiar straightening of the feather is visible. Additional sweep is visible under load, though this too appears gradually over the span of the feather.

Clearly, the primary feathers of the Brown Pelican exhibit significant flex under aerodynamic loading. While the aerodynamic test model that was constructed exhibits virtually no flex, other 3D printing materials exist that may allow flex similar to that of a Brown Pelican feather while maintaining structural rigidity. Alternative prototyping methods could also be considered. Future studies will need to be conducted if more accurate test models are to be constructed. 


\section{B. Engineering Drawings}

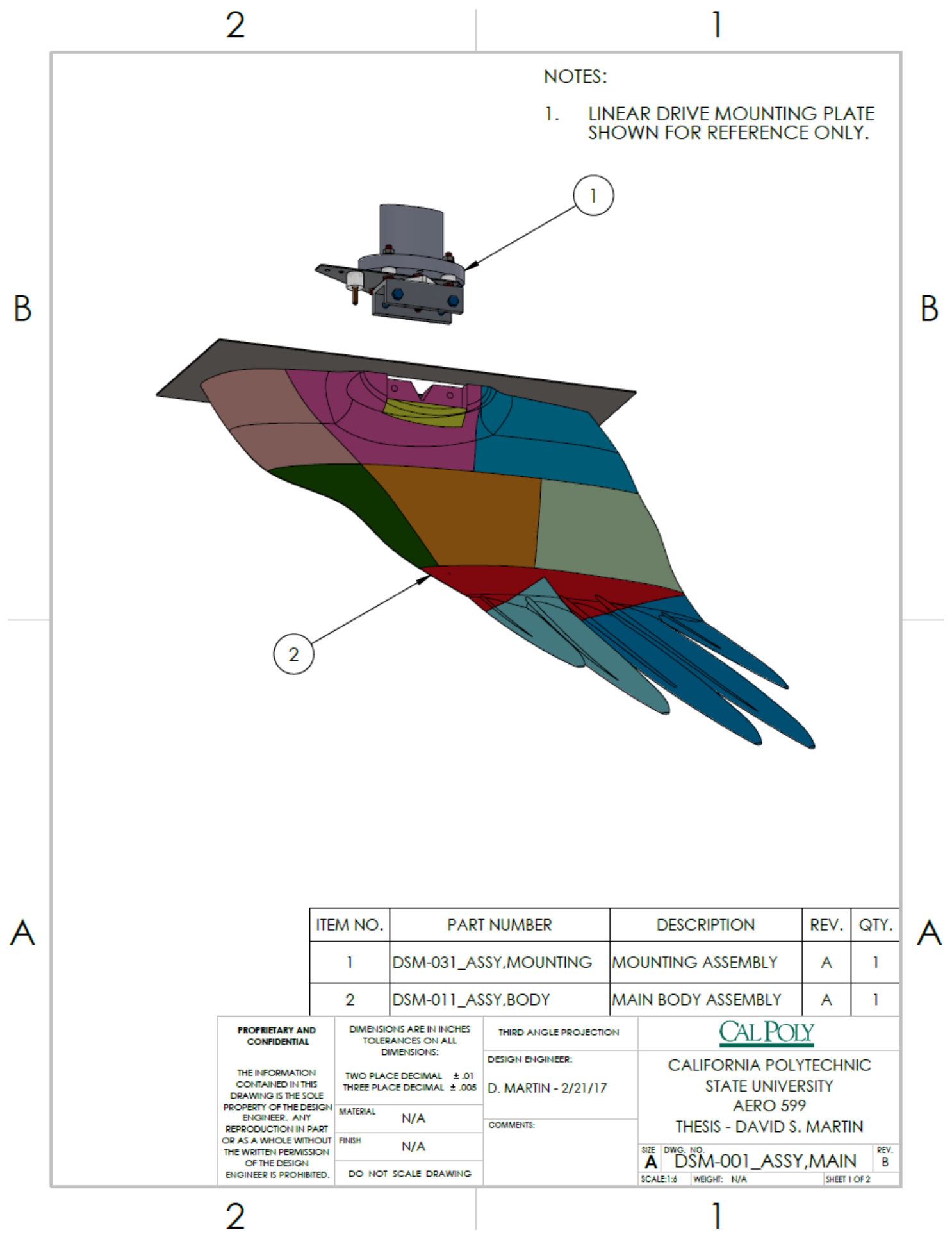




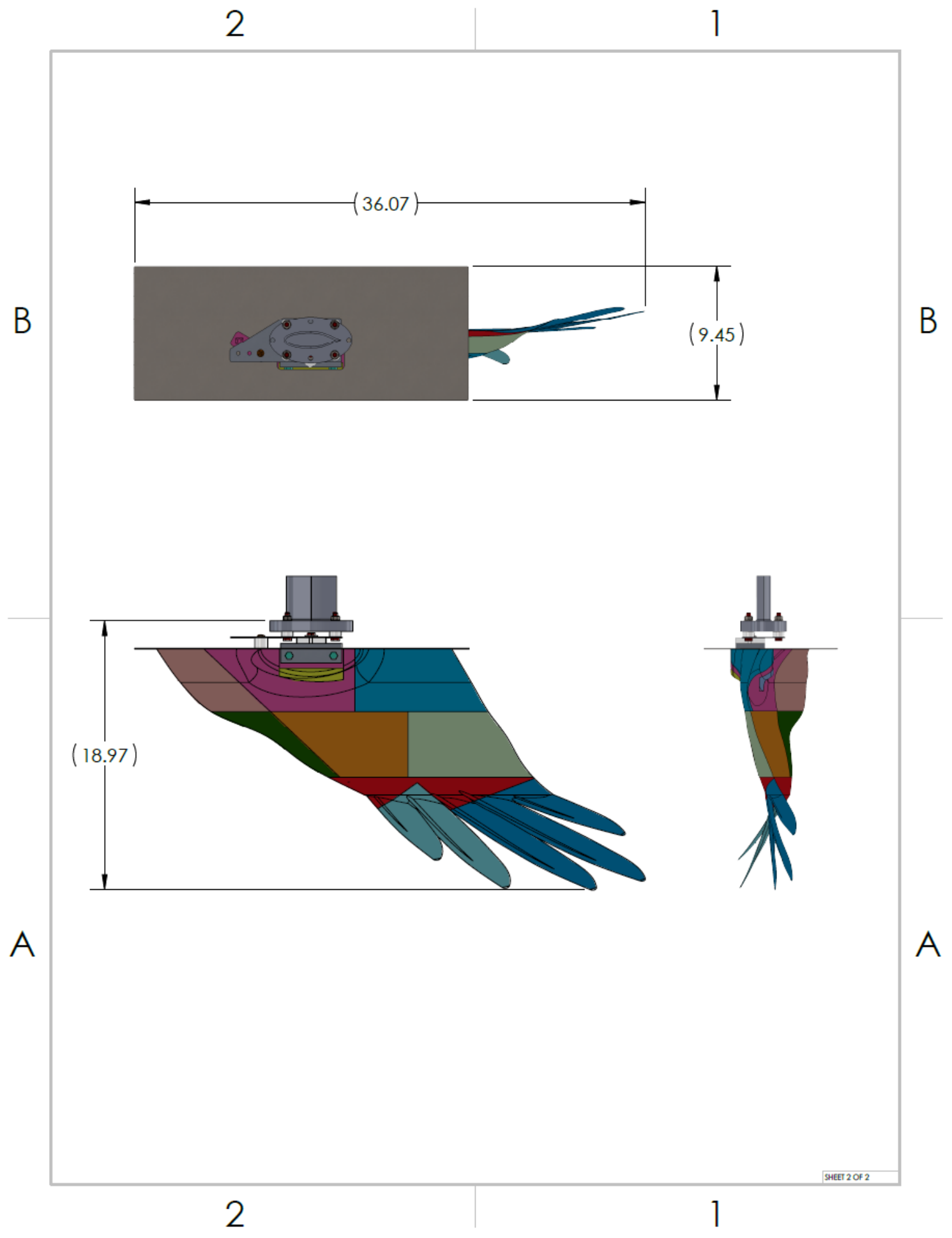




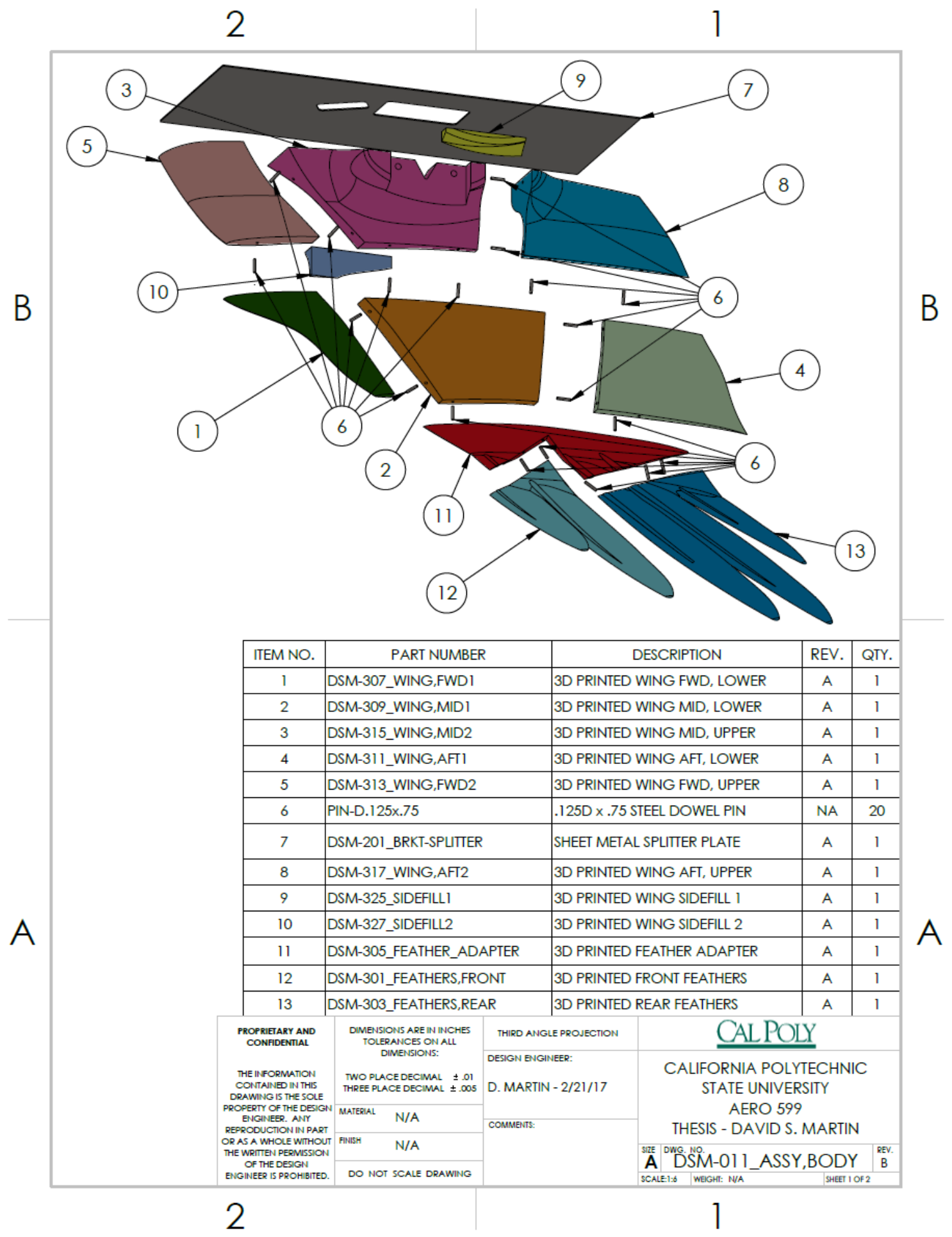




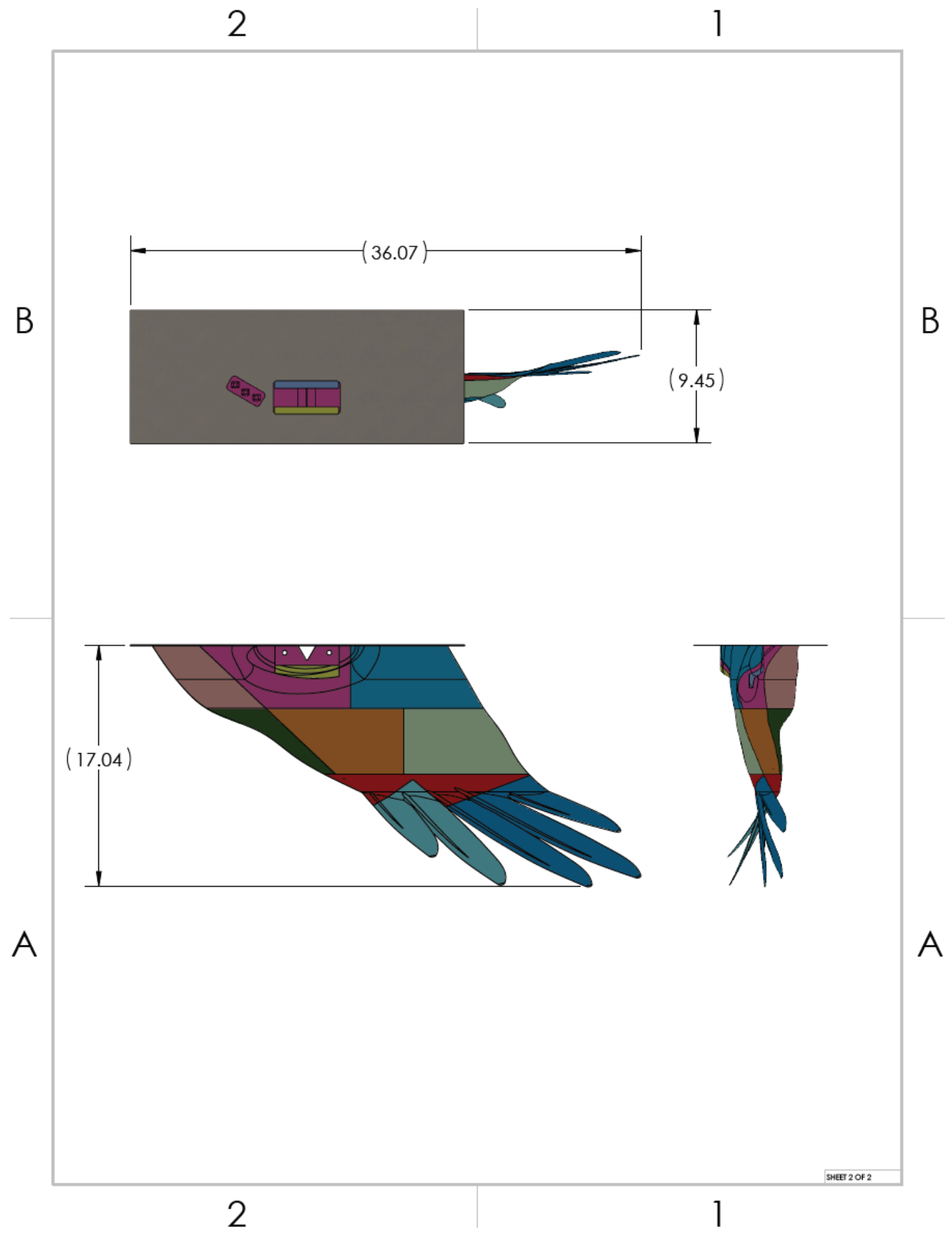




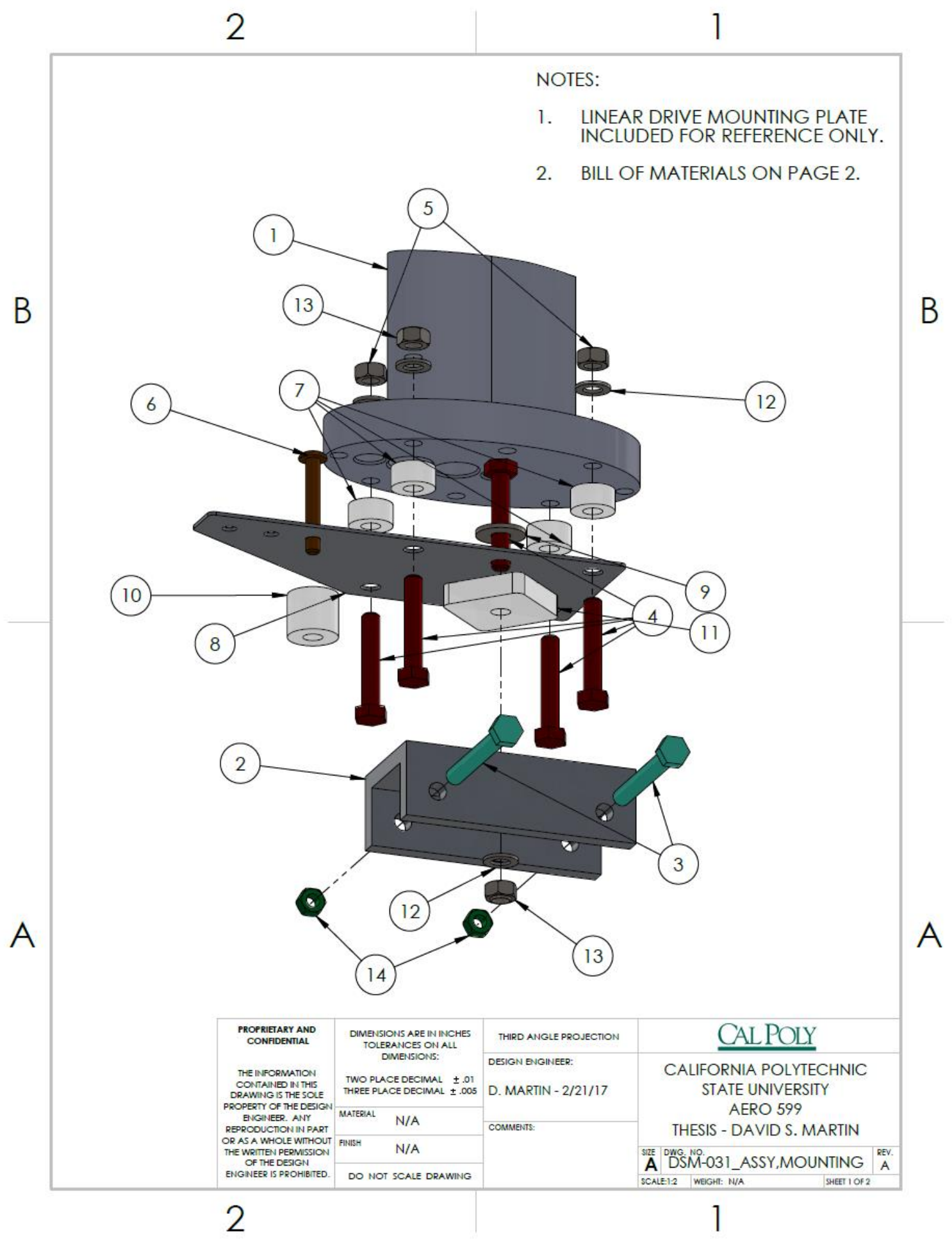




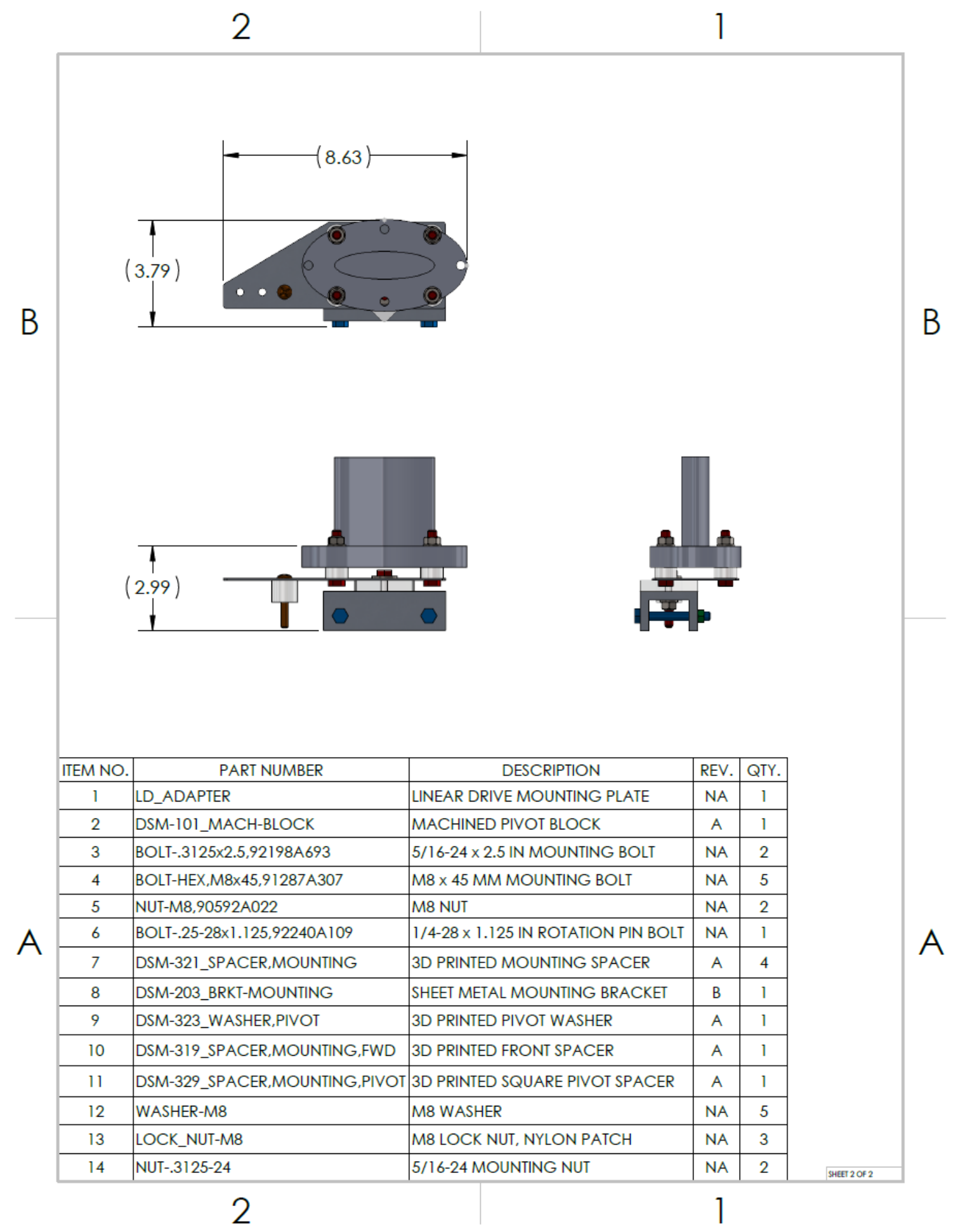




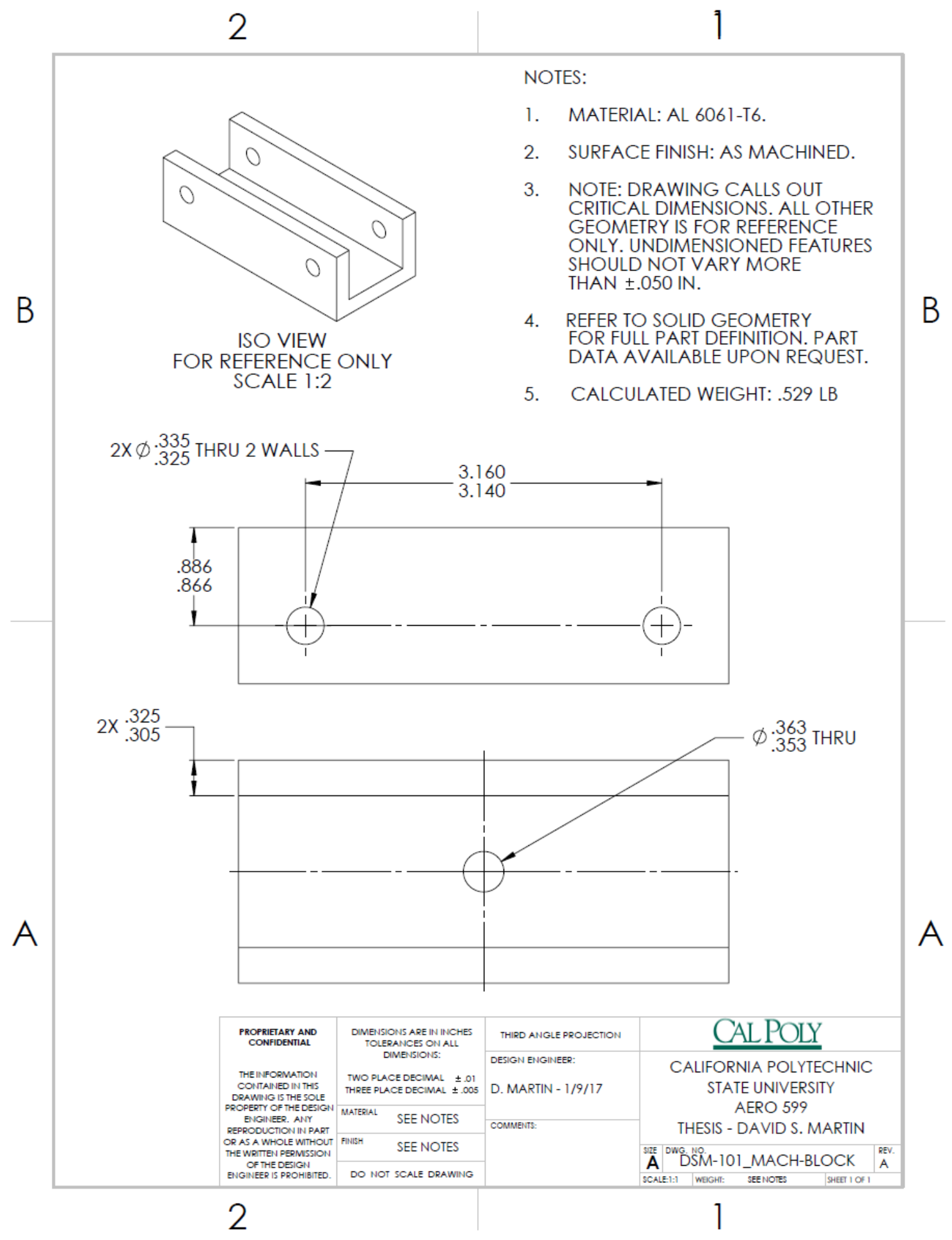




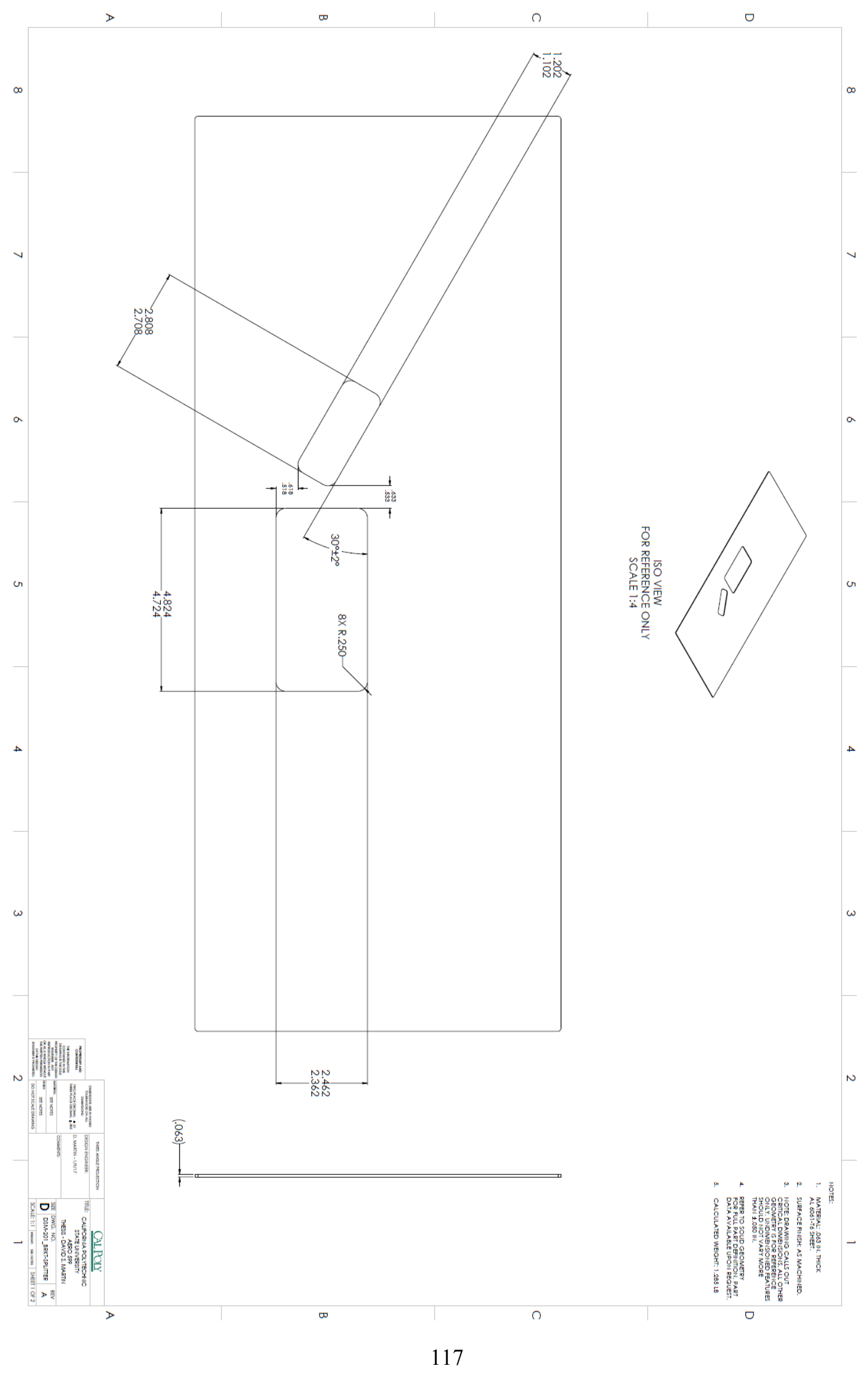




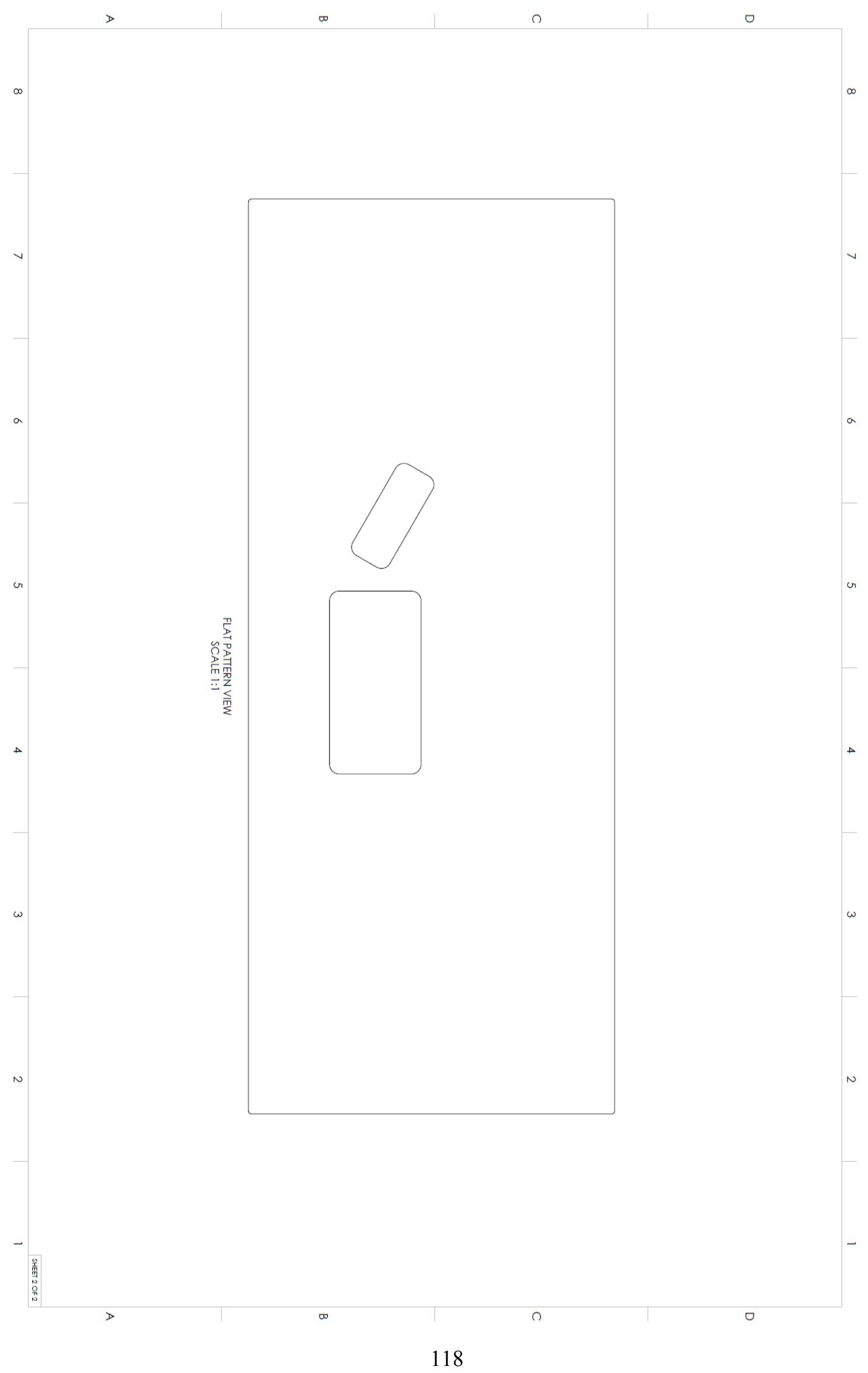




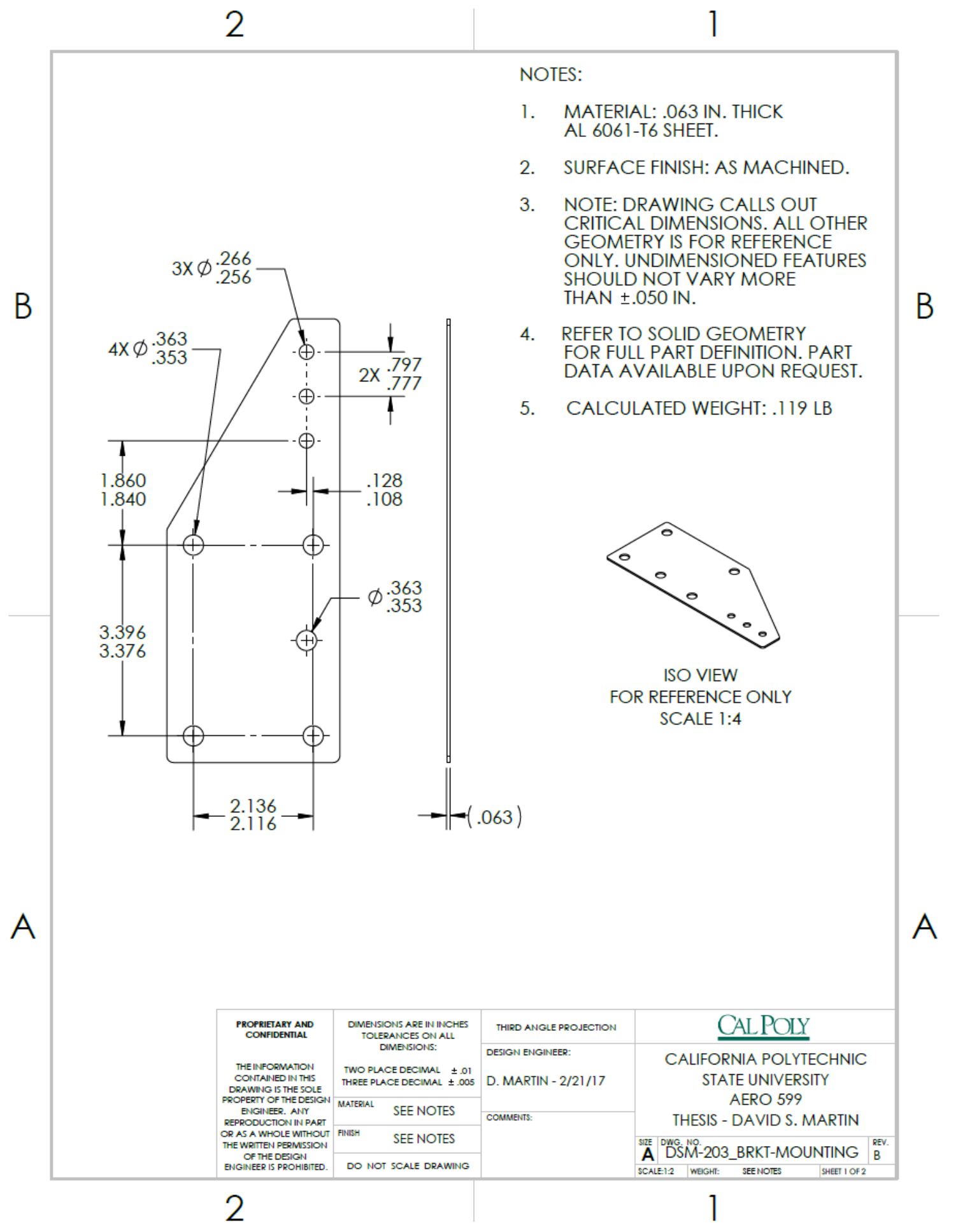




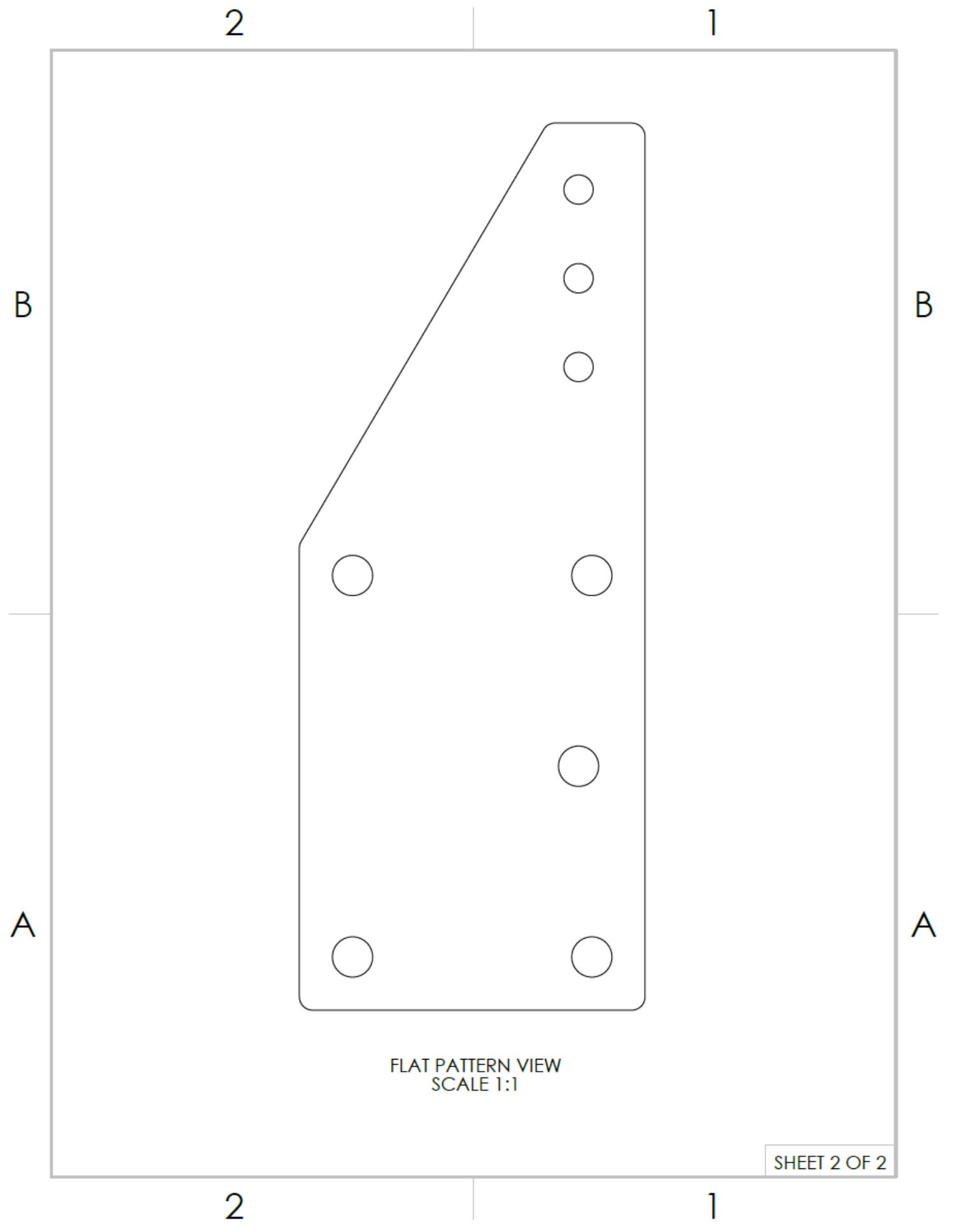




\section{Sectioner3D MATLAB Script}

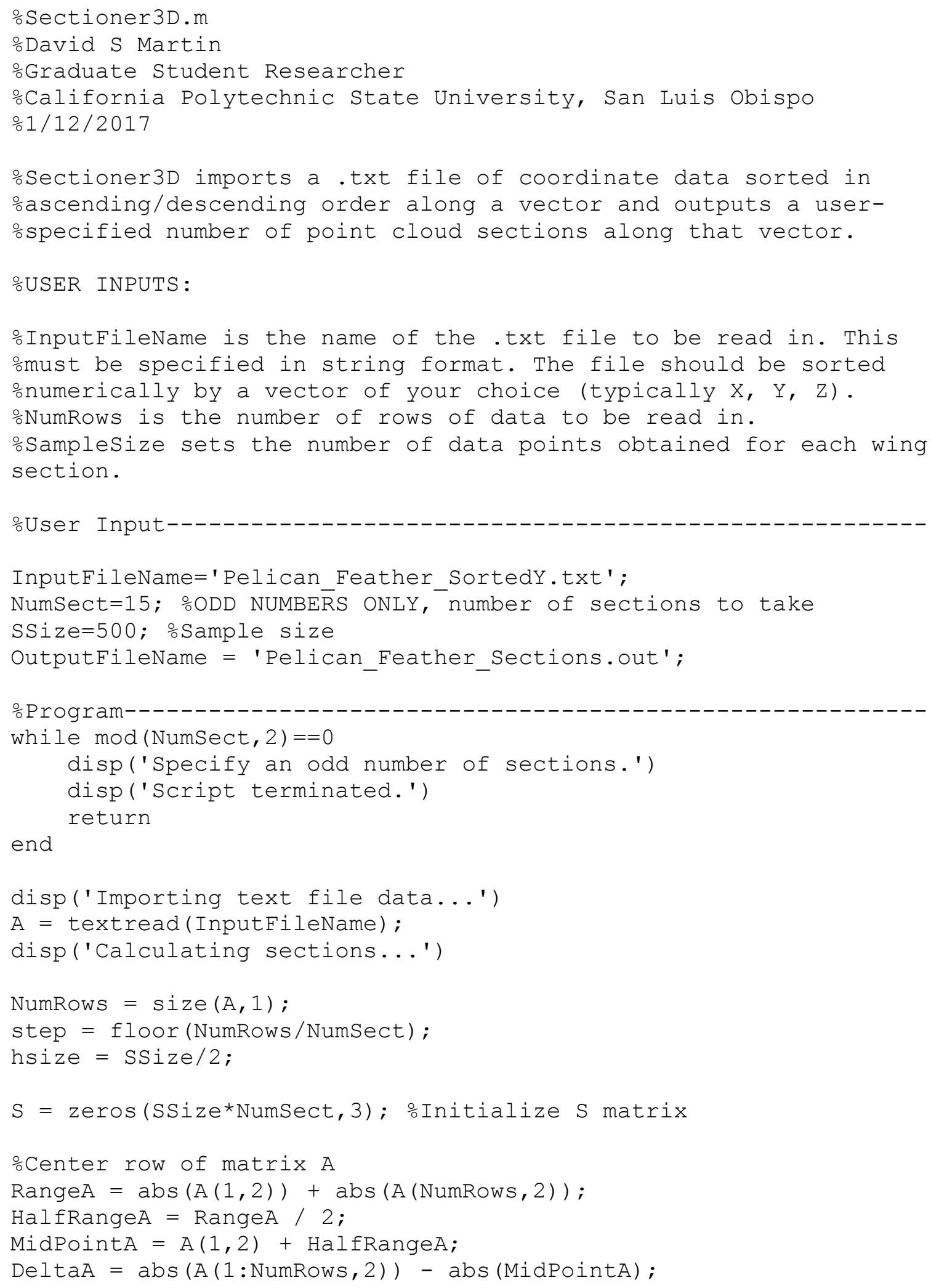


CenterRowA $=$ knnsearch $(\operatorname{DeltaA}, 0) ; \%$ Find the data row corresponding to the geometric center of the object

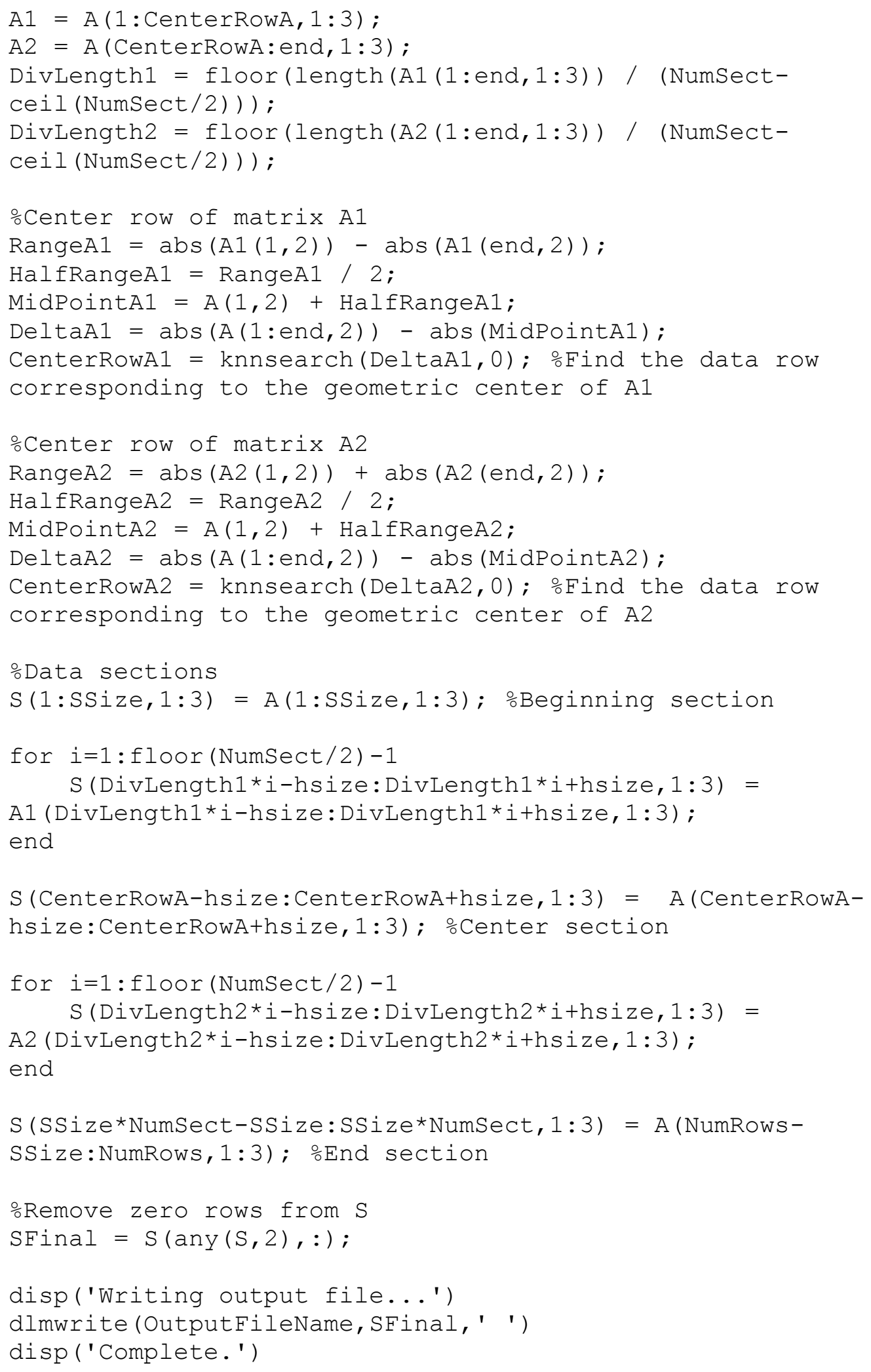




\section{Total Pressure Rake Post-Processing MATLAB Script}

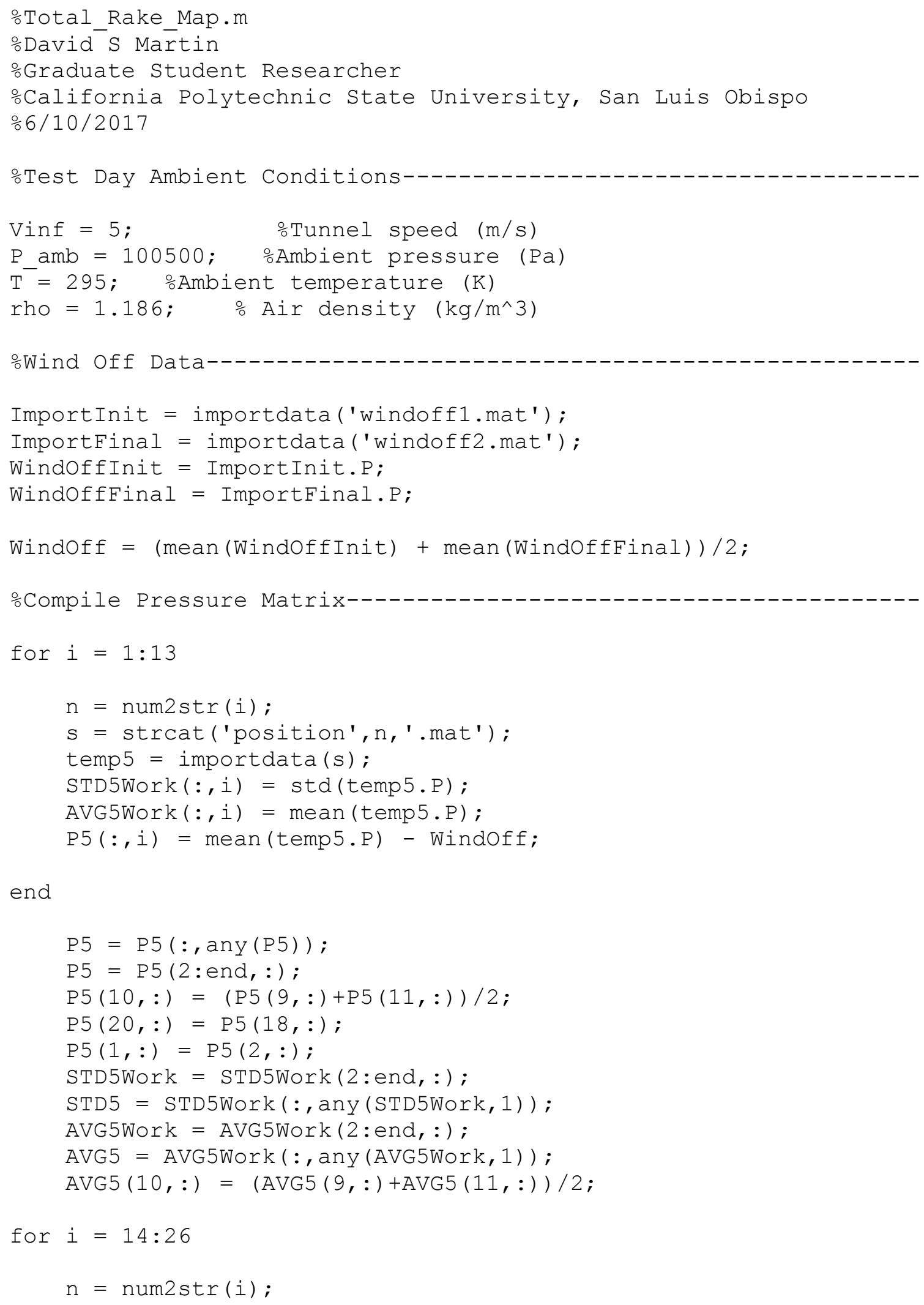

end 
$\mathrm{s}=\operatorname{strcat}($ 'position',n, '.mat');

temp4 = importdata (s);

$\operatorname{STD} 4$ Work $(:, i)=\operatorname{std}($ temp $4 . P)$;

$\operatorname{AVG} 4$ Work $(:, i)=\operatorname{mean}($ temp $4 . P)$;

$\mathrm{P} 4(:, i)=$ mean $($ temp $4 . \mathrm{P})-$ Windoff;

end

$\mathrm{P} 4=\mathrm{P} 4(:, \operatorname{any}(\mathrm{P} 4)) ;$

$\mathrm{P} 4=\mathrm{P} 4(2:$ end,$:)$;

$\mathrm{P} 4(10,:)=(\mathrm{P} 4(9,:)+\mathrm{P} 4(11,:)) / 2$;

$\mathrm{P} 4(20,:)=\mathrm{P} 5(18,:)$;

$\mathrm{P} 4(1,:)=\operatorname{P} 4(2,:)$;

STD4Work = STD4Work (2:end, : );

STD4 = STD4Work $(:, \operatorname{any}(\operatorname{STD} 4$ Work, 1$))$;

AVG4Work = AVG4Work (2:end, : );

AVG4 = AVG4Work $(:$, any $($ AVG4Work, 1$))$;

$\operatorname{AVG} 4(10,:)=(\operatorname{AVG} 4(9,:)+\operatorname{AVG} 4(11,:)) / 2$;

for $i=27: 39$

$\mathrm{n}=$ num2str(i);

$\mathrm{s}=$ strcat ('position',n,'.mat');

temp3 = importdata $(\mathrm{s})$;

$\operatorname{STD} 3$ Work $(:$, i $)=\operatorname{std}($ temp3.P $)$;

$\operatorname{AVG} 3$ Work $(:, i)=\operatorname{mean}($ temp3.P) ;

$\mathrm{P} 3(:, i)=$ mean $($ temp3.P $)-$ Windoff;

end

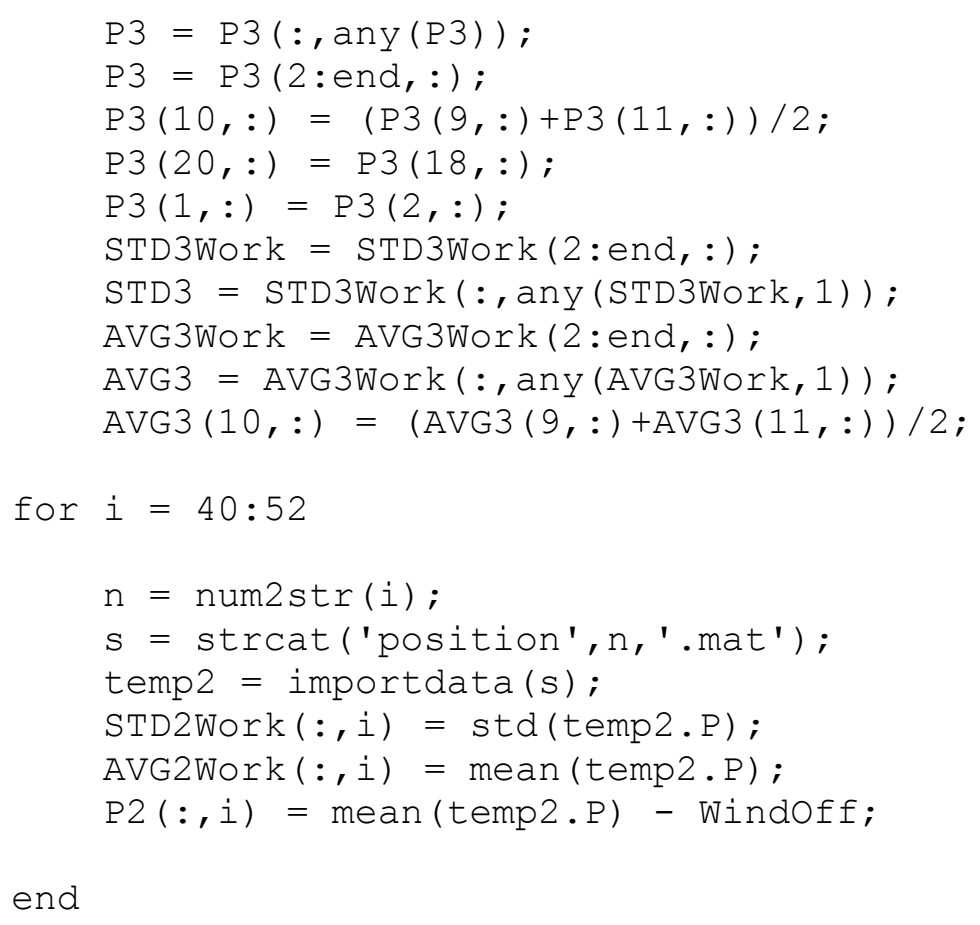

end 


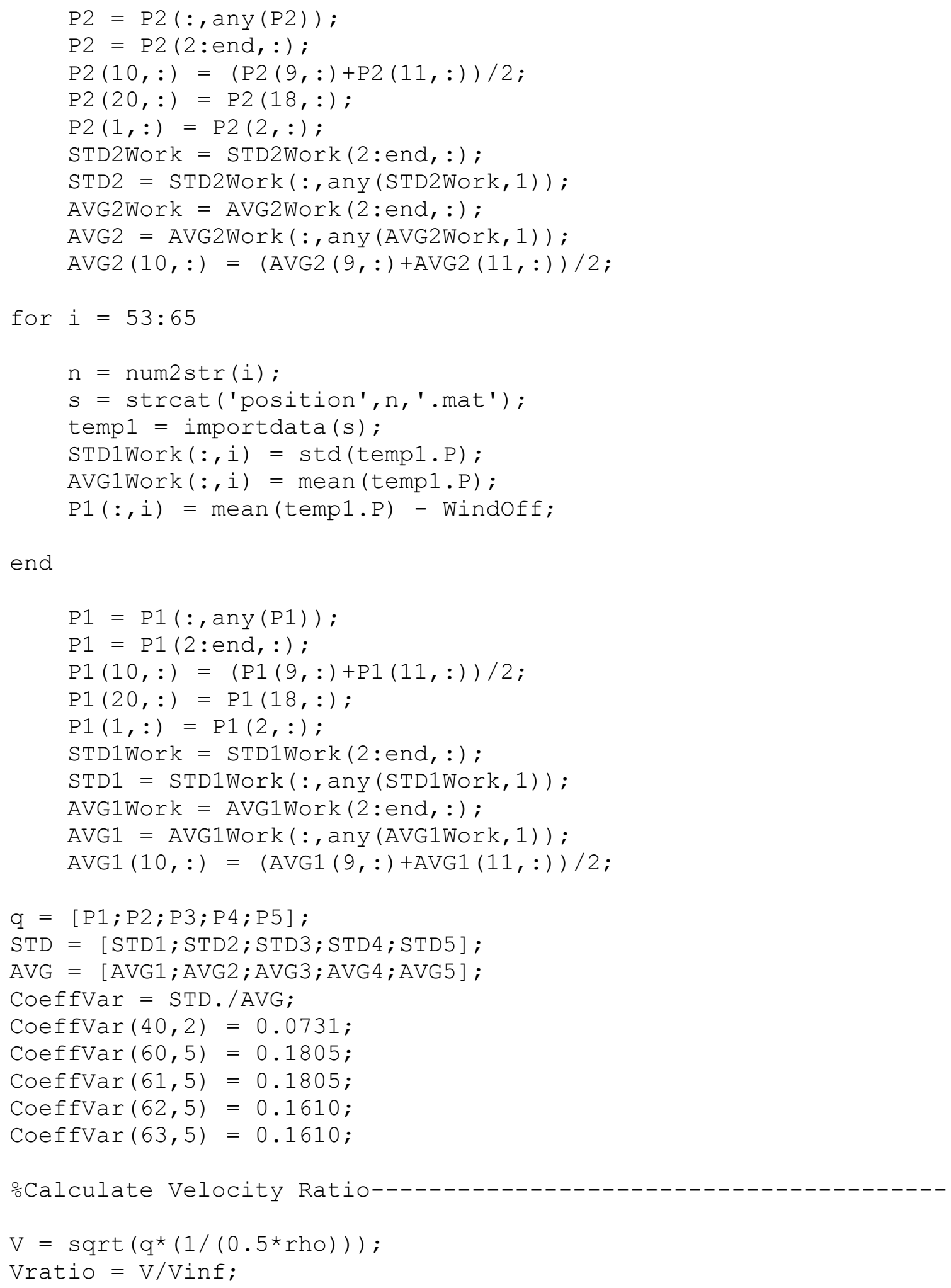

end 


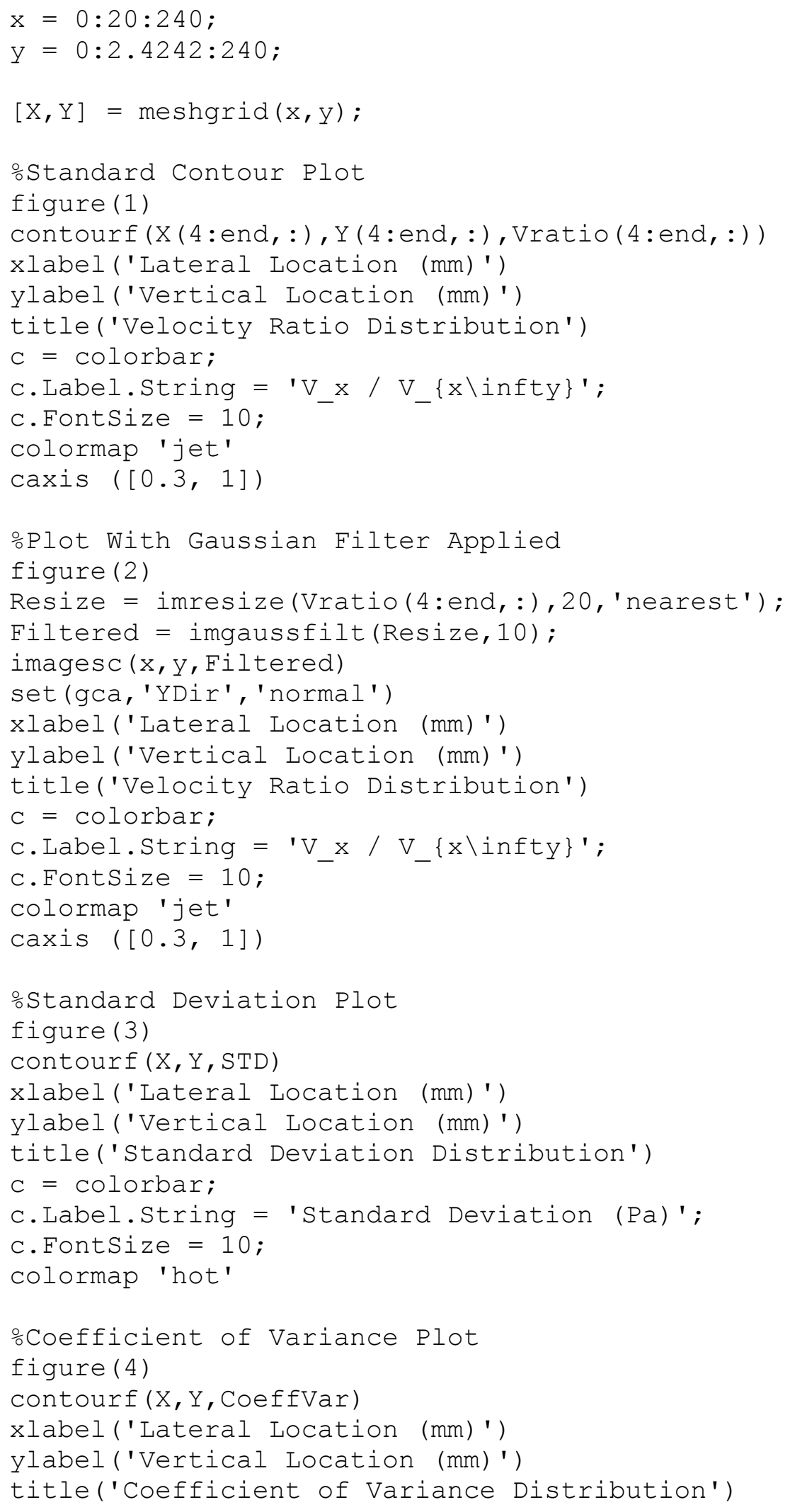


$\mathrm{c}=$ colorbar;

c.Label.String = 'Coefficient of Variance';

c.FontSize = 10;

colormap 'cool' 


\section{E. Total Pressure Probe Post-Processing MATLAB Script - Example}

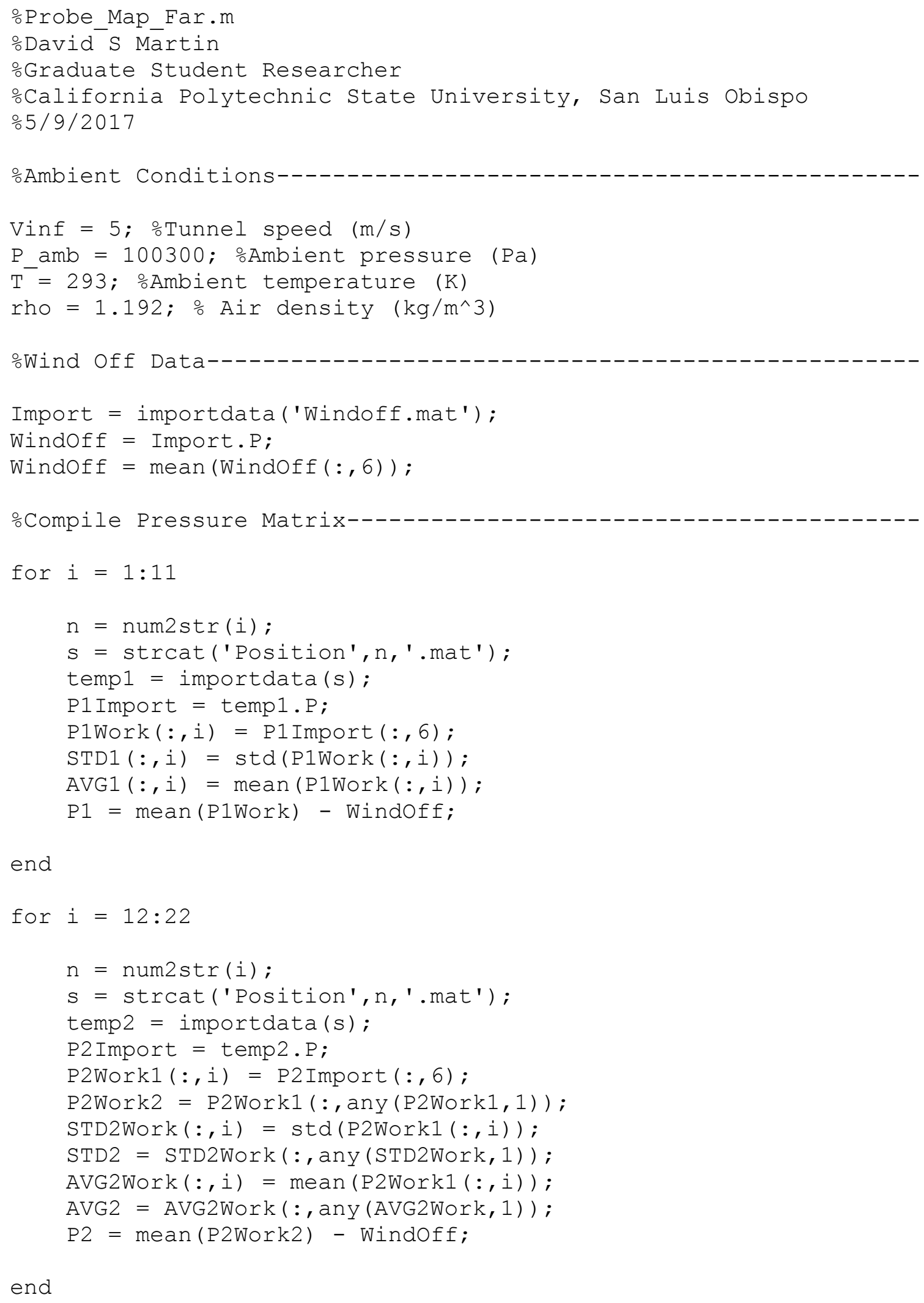

end 
for $i=23: 33$

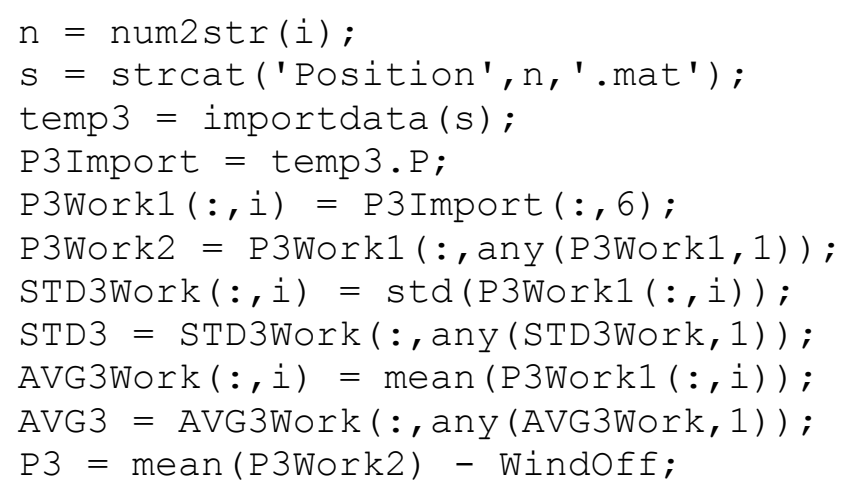

end

for $i=34: 44$

$\mathrm{n}=$ num2str $(\mathrm{i})$;

$\mathrm{s}=$ strcat ('Position',n,'.mat');

temp4 = importdata (s);

P4 Import $=$ temp4.P;

P4Work1 $(:, i)=P 4 \operatorname{Import}(:, 6)$;

P4Work2 = P4Work1 (:, any $(P 4$ Work1, 1$))$; $\operatorname{STD} 4$ Work $(:, i)=\operatorname{std}(\operatorname{P} 4 \operatorname{Work} 1(:, i))$; $\operatorname{STD} 4=\operatorname{STD} 4$ Work $(: \operatorname{any}(\operatorname{STD} 4$ Work, 1$))$; $\operatorname{AVG} 4 \operatorname{Work}(:$, i $)=\operatorname{mean}(\operatorname{P} 4 \operatorname{Work} 1(:, i))$; AVG4 = AVG4Work $(: \operatorname{any}($ AVG4Work, 1$))$; $\mathrm{P} 4=$ mean (P4Work2) - WindOff;

end

for $i=45: 55$

$\mathrm{n}=$ num2str(i);

$\mathrm{s}=$ strcat ('Position',n,'.mat');

temp5 = importdata (s);

P5Import $=$ temp5.P;

$\operatorname{P5Work1}(:, i)=\operatorname{P5Import}(:, 6)$;

P5Work2 = P5Work1 $(:$, any $($ P 5 Work1, 1$))$; $\operatorname{STD} 5 \operatorname{Work}(:, i)=\operatorname{std}(\operatorname{PWWork1}(:, i))$; $\operatorname{STD5}=\operatorname{STD} 5$ Work $(: \operatorname{any}(\operatorname{STD} 5$ Work, 1$))$; $\operatorname{AVG} 5 \operatorname{Work}(:$, i $)=\operatorname{mean}(\operatorname{P5Work} 1(:, i))$; AVG5 = AVG5Work $(: \operatorname{any}($ AVG5Work, 1)); $\mathrm{P} 5=$ mean (P5Work2) - Windoff;

end

for $i=56: 66$ 


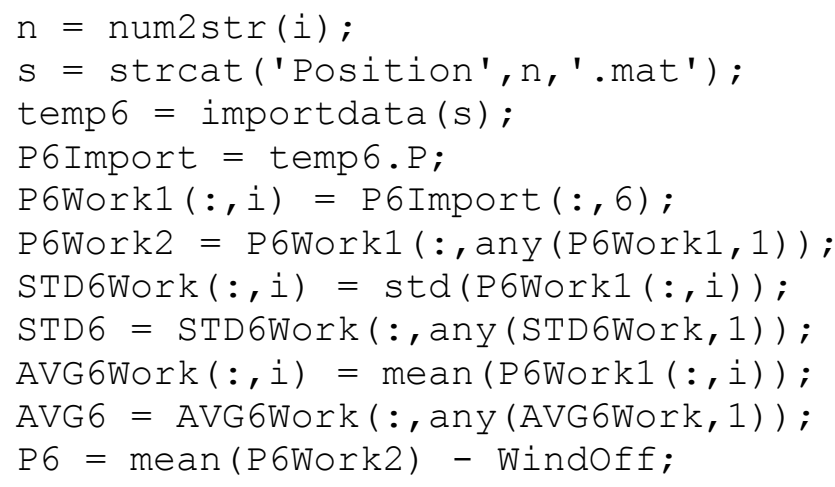

end

for $i=67: 77$

$\mathrm{n}=\operatorname{num} 2 \mathrm{str}(\mathrm{i})$;

$\mathrm{s}=$ strcat('Position', n,' 'mat');

temp $7=$ importdata $(\mathrm{s})$;

P7Import $=$ temp $7 . \mathrm{P}$;

P7Work1 $(:, i)=\operatorname{P7Import~}(:, 6)$;

P7Work2 = P7Work1 (:, any $($ P7Work1, 1));

$\operatorname{STD} 7 \operatorname{Work}(:, i)=\operatorname{std}(\operatorname{P7Work1}(:, i))$;

STD7 = STD7Work $(: \operatorname{any}(\operatorname{STD} 7$ Work, 1$))$;

$\operatorname{AVG} 7 \operatorname{Work}(:, i)=\operatorname{mean}(\operatorname{P} 7 \operatorname{Work} 1(:, i))$;

AVG7 = AVG7Work $(: \operatorname{any}($ AVG7Work, 1));

$\mathrm{P} 7=\operatorname{mean}(\mathrm{P} 7$ Work2) - Windoff;

end

for $i=78: 88$

$\mathrm{n}=\operatorname{num} 2 \mathrm{str}(\mathrm{i}) ;$

$\mathrm{s}=$ strcat ('Position', $\mathrm{n}$, '.mat');

temp $8=$ importdata $(\mathrm{s})$;

P8Import $=$ temp8.P;

P8Work1 $(:, i)=\operatorname{P} 8 \operatorname{Import}(:, 6)$;

P8Work2 = P8Work1 (: , any $($ P8Work1, 1)); $\operatorname{STD} 8 W o r k(:, i)=\operatorname{std}(\operatorname{PoWork} 1(:, i))$; $\operatorname{STD} 8=\operatorname{STD} 8 W o r k(: \operatorname{any}(\operatorname{STD} 8 W o r k, 1))$; $\operatorname{AVG} 8 \operatorname{Work}(:, i)=\operatorname{mean}(\operatorname{PBWork} 1(:, i))$; AVG8 = AVG8Work $(: \operatorname{any}(\operatorname{AVG} 8$ Work, 1$))$; $\mathrm{P} 8=\operatorname{mean}(\mathrm{P} 8$ Work2) - Windoff;

end

for $i=89: 99$

$\mathrm{n}=\operatorname{num} 2 \mathrm{str}(\mathrm{i}) ;$

$\mathrm{s}=$ strcat('Position', $\mathrm{n}$, '.mat');

temp $9=$ importdata $(\mathrm{s})$; 


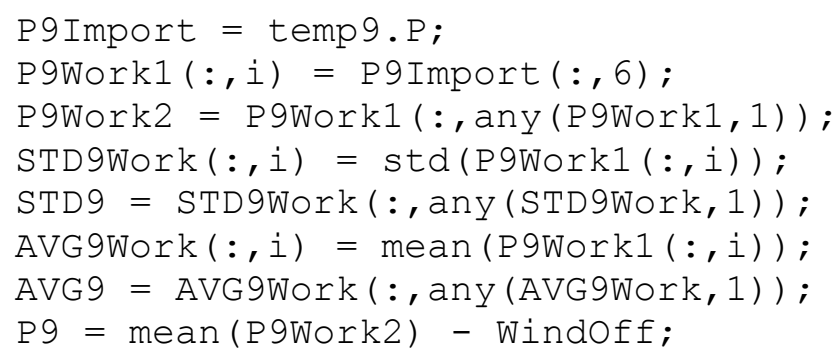

end

for $i=100: 110$

$\mathrm{n}=$ num2str(i);

$\mathrm{s}=$ strcat ('Position',n,'.mat');

temp10 = importdata (s);

P10 Import = temp10.P;

P10Work1 $(:, i)=\operatorname{P10Import}(:, 6)$;

P10Work2 = P10Work1 (:, any (P10Work1, 1));

$\operatorname{STD10Work}(:, i)=\operatorname{std}(\operatorname{P1OWork1}(:, i))$;

$\operatorname{STD10}=\operatorname{STD10Work}(: \operatorname{any}(\operatorname{STD} 10$ Work, 1$))$;

$\operatorname{AVG10Work}(:, i)=\operatorname{mean}(\operatorname{P1OWork} 1(:, i))$;

$\operatorname{AVG10}=\operatorname{AVG10Work}(:$, any $($ AVG10Work, 1$))$;

$\mathrm{P} 10=$ mean $(\mathrm{P} 10$ Work2) - Windoff;

end

for $i=111: 121$

$\mathrm{n}=$ num2str $(\mathrm{i})$;

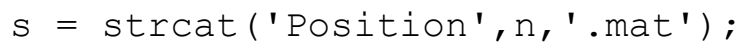

temp11 = importdata $(\mathrm{s})$;

P11 Import $=$ temp11.P;

P11Work1 $(:, i)=\operatorname{P} 11$ Import $(:, 6)$;

P11Work2 = P11Work1 (:, any $(\mathrm{P} 11$ Work1, 1$))$;

$\operatorname{STD} 11 \operatorname{Work}(:, i)=\operatorname{std}(\operatorname{P11Work1}(:, i))$;

STD11 = STD11Work $(:$, any $($ STD11Work, 1$))$;

$\operatorname{AVG11Work}(:, i)=\operatorname{mean}(\operatorname{P1} 1 \operatorname{Work} 1(:, i))$;

AVG11 = AVG11Work $(:$, any (AVG11Work, 1));

$\mathrm{P} 11$ = mean (P11Work2) - WindOff;

end

$\mathrm{q}=[\mathrm{P} 11 ; \mathrm{P} 10 ; \mathrm{P} 9 ; \mathrm{P} 8 ; \mathrm{P} 7 ; \mathrm{P} 6 ; \mathrm{P} 5 ; \mathrm{P} 4 ; \mathrm{P} 3 ; \mathrm{P} 2 ; \mathrm{P} 1] ;$

$\mathrm{STD}=[\mathrm{STD} 11 ; \mathrm{STD} 10 ; \mathrm{STD} 9 ; \mathrm{STD} 8 ; \mathrm{STD} 7 ; \mathrm{STD} 6 ; \mathrm{STD} 5 ; \mathrm{STD} 4 ; \mathrm{STD} 3 ; \mathrm{STD} 2 ; \mathrm{STD} 1] ;$

$\mathrm{AVG}=[\mathrm{AVG} 11 ; \mathrm{AVG} 10 ; \mathrm{AVG} 9 ; \mathrm{AVG} 8 ; \mathrm{AVG} 7 ; \mathrm{AVG} 6 ; \mathrm{AVG} 5 ; \mathrm{AVG} 4 ; \mathrm{AVG} 3 ; \mathrm{AVG} 2 ; \mathrm{AVG} 1]$;

CoeffVar = STD./AVG; 


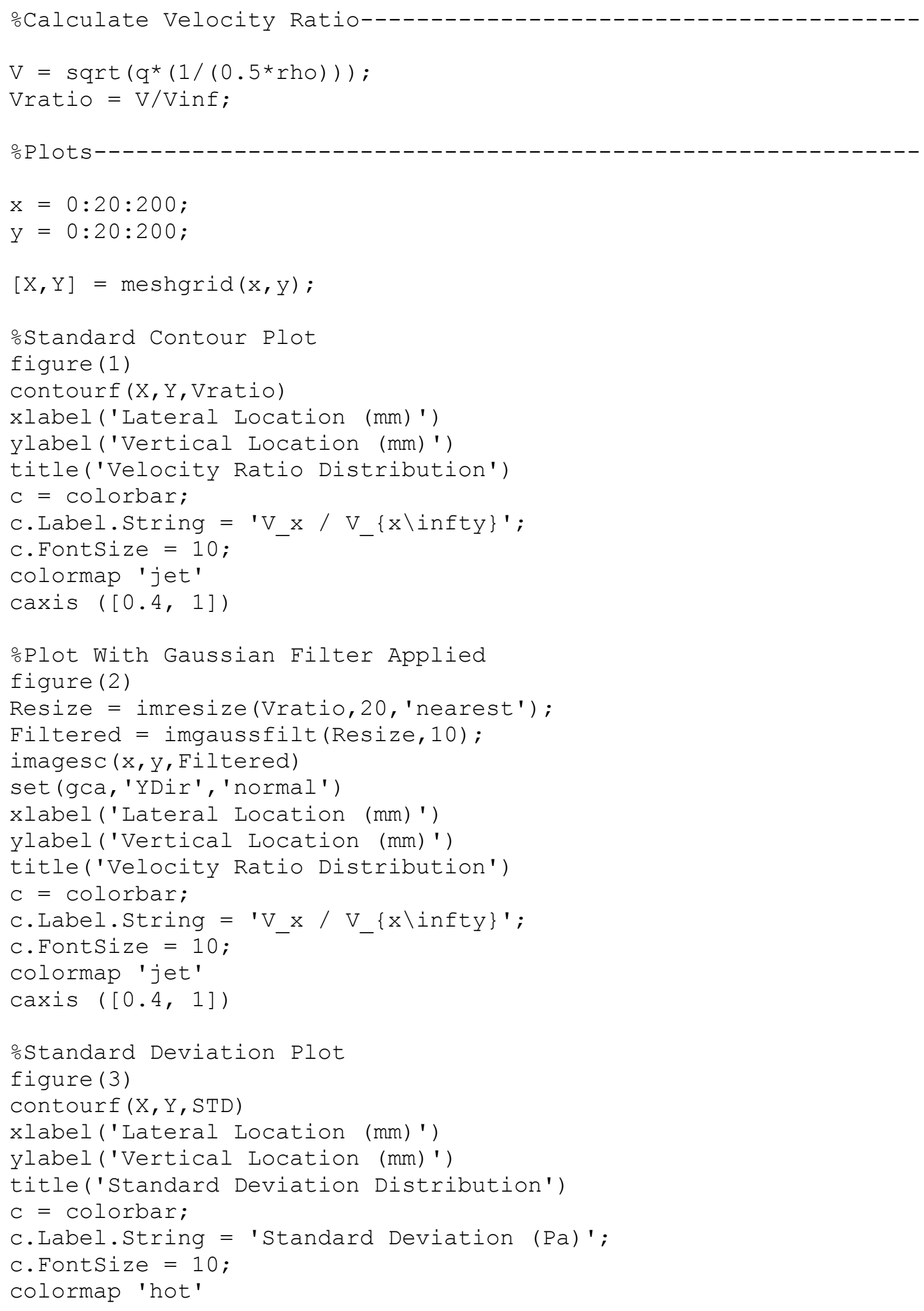


oCoefficient of Variance Plot

figure (4)

contourf (X, Y, CoeffVar)

xlabel ('Lateral Location (mm)')

ylabel ('Vertical Location (mm)')

title('Coefficient of Variance Distribution')

$\mathrm{c}=$ colorbar;

c.Label.String = 'Coefficient of Variance';

c. FontSize = 10;

colormap 'cool' 